\title{
En(Gulf)ed: Frame Analysis of New York Times Online News Coverage following the 2010 BP Oil Spill
}

by

\author{
Holly Gilroy
}

A thesis submitted to the Faculty of Graduate and Postdoctoral Affairs in partial fulfillment of the requirements for the degree of

Master of Arts

in

Sociology

Carleton University

Ottawa, Ontario

(C) 2011, Holly Gilroy 


$\begin{array}{ll}\begin{array}{l}\text { Library and Archives } \\ \text { Canada }\end{array} & \begin{array}{l}\text { Bibliotheque et } \\ \text { Archives Canada }\end{array} \\ \begin{array}{l}\text { Published Heritage } \\ \text { Branch }\end{array} & \begin{array}{l}\text { Direction du } \\ \text { Patrimoine de l'édition }\end{array} \\ \begin{array}{l}\text { 395 Wellington Street } \\ \text { Ottawa ON K1A ON4 } \\ \text { Canada }\end{array} & \begin{array}{l}\text { 395, rue Wellington } \\ \text { Ottawa ON K1A ON4 } \\ \text { Canada }\end{array}\end{array}$

Your file Votre référence

ISBN: 978-0-494-83088-8

Our file Notre référence

ISBN: $978-0-494-83088-8$

NOTICE:

AVIS:

The author has granted a nonexclusive license allowing Library and Archives Canada to reproduce, publish, archive, preserve, conserve, communicate to the public by telecommunication or on the Internet, loan, distribute and sell theses worldwide, for commercial or noncommercial purposes, in microform, paper, electronic and/or any other formats.

The author retains copyright ownership and moral rights in this thesis. Neither the thesis nor substantial extracts from it may be printed or otherwise reproduced without the author's permission.

L'auteur a accordé une licence non exclusive permettant à la Bibliothèque et Archives Canada de reproduire, publier, archiver, sauvegarder, conserver, transmettre au public par télécommunication ou par l'internet, prêter, distribuer et vendre des thèses partout dans le monde, à des fins commerciales ou autres, sur support microforme, papier, électronique et/ou autres formats.

L'auteur conserve la propriété du droit d'auteur et des droits moraux qui protège cette thèse. $\mathrm{Ni}$ la thèse ni des extraits substantiels de celle-ci ne doivent être imprimés ou autrement reproduits sans son autorisation.
In compliance with the Canadian Privacy Act some supporting forms may have been removed from this thesis.

While these forms may be included in the document page count, their removal does not represent any loss of content from the thesis.
Conformément à la loi canadienne sur la protection de la vie privée, quelques formulaires secondaires ont été enlevés de cette thèse.

Bien que ces formulaires aient inclus dans la pagination, il n'y aura aucun contenu manquant.

\section{Canadä}




\begin{abstract}
This study examines New York Times online news coverage after the Deepwater Horizon oil platform sank in the Gulf of Mexico in 2010. I utilize literatures on the rise of neoliberalism, the political economy of news, sociology of news production, and media and environment. I suggest that the coverage did not connect the spill to economic context or a structural critique and many sources framed it as an isolated accident. However, the spill can be linked to economic policy that prioritizes profit over human or environmental health. Analysis of a sample of New York Times reports identifies practices inhibiting the presentation of in-depth structural economic issues, notably journalists' reliance on authoritative sources, especially BP and government. I employ frame analysis to consider the media representation of key stakeholders in the disaster. I determine that dominant frames reflect neoliberal ideology evidenced through individualization, promotion of business solutions and omission of alternate frames.
\end{abstract}




\section{Acknowledgements}

I owe my presence in academia almost exclusively to the kindness and generosity of my mentors and supervisors, who all along this journey have done everything they can to convince me I could succeed in grad school. I feel heartfelt gratitude toward this group of patient, giving women and men who have always gone above and beyond to provide support.

This project was only possible through the thoughtful guidance of my two exceptional supervisors, Dr. Aaron Doyle and Dr. Janet Siltanen. They provided constant confidence, support and positivity in the face of a difficult and slow project. I am deeply grateful for our meetings and emails as a source of calm during an anxious time.

I owe my participation in academia to the mentoring, patience and commitment of Dr. Susan Machum who guided me, step-by-step through my honours thesis and into graduate studies. I would never have believed I could do this without Susan's encouragement and willingness to commit her time and energies to my success. The teaching of Dr. Peter Weeks inspired my interest in Sociology and media studies. Along with Susan, he graciously helped me through my undergraduate degree,

Thank you to my wonderful peers Rebecca, Jen, Lisa, Jordan, Alana, Caryn and Emerich for the coffee dates, the food, and the conversations. Thank you to my comrades at the GSA for the unyielding patience while I stumbled through this process. Thank you to my family, specifically my brilliant sister who gave up countless precious hours to edit this project. Finally, thank you Matt, whose care, loyalty and unwavering belief that I was more than capable meant the world. 


\section{Table of Contents}

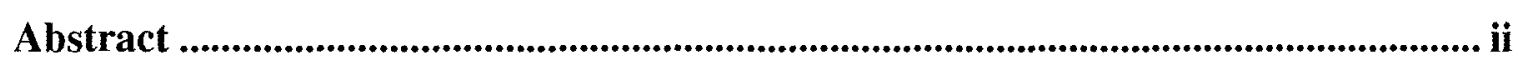

Acknowledgements ....................................................................................................................... iii

Table of Contents ................................................................................................................ iv

List of Appendices.................................................................................................................. vi

I Chapter One: Introduction .......................................................................................................1

\section{Chapter Two: Theoretical Context: Sociology of News Media and Political}

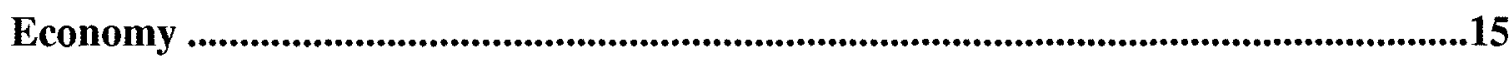

$2.1 \quad$ Political Economy and Neoliberalism ................................................................. 16

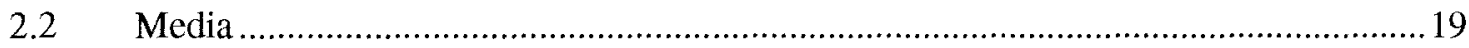

2.3 Comparing Perspectives in Sociology of News Production ........................................31

$2.4 \quad$ Key Concepts: Frames, Ideology, Discourse …………...............................................

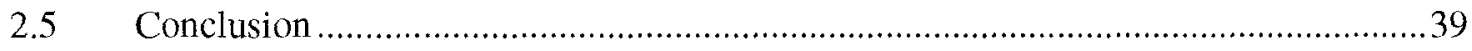

III Chapter Three: Designing A Methodological Approach To Studying News ....41

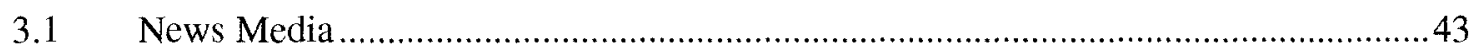

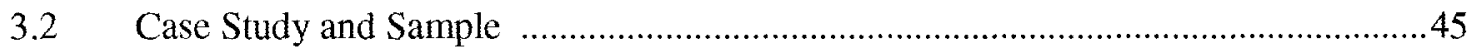

$3.3 \quad$ Voice and Access.........................................................................................52

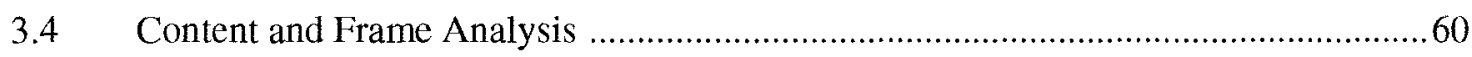

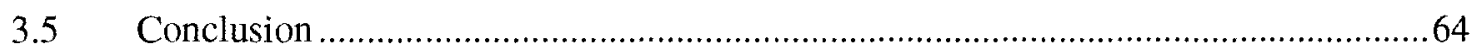

IV Chapter Four: Voice and Access................................................................................65

4.1 Overview of the Categories ....................................................................................6

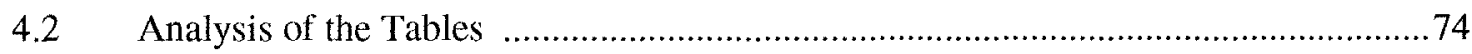

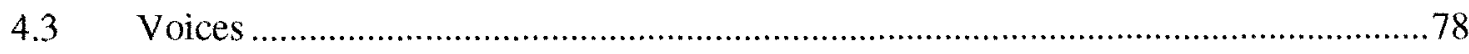

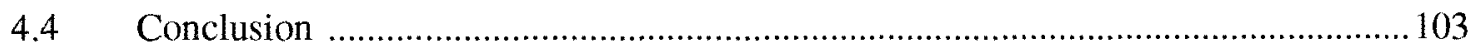


V Chapter Five: Frames and Themes..........................................................................106

5.1 Individualizes Coverage and a Focuses on the Exceptional .................................. 108

5.2 Prioritizing Business and the Free Market:........................................................ 121

5.3 Minimizes Magnitude .............................................................................. 137

5.4 Marginalized and Omitted Frames ................................................................ 143

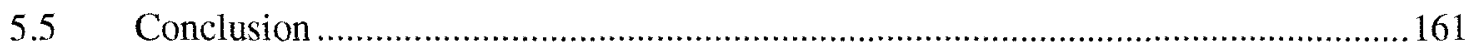

VI Chapter Six: Conclusion ...............................................................................165

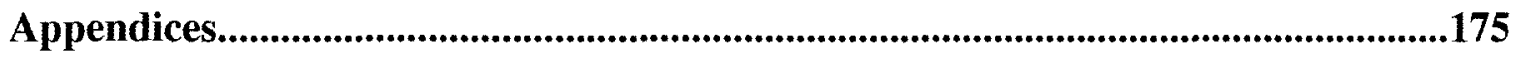

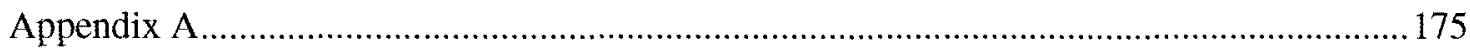

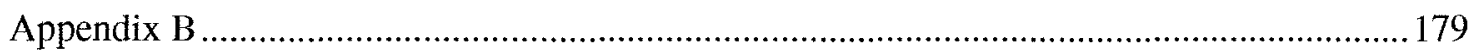

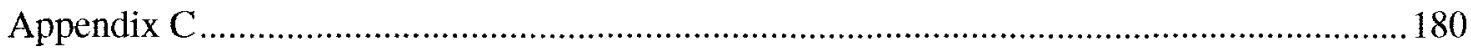

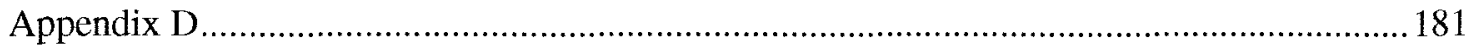

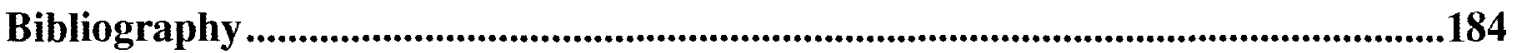




\section{List of Appendices}

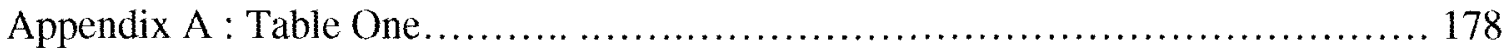

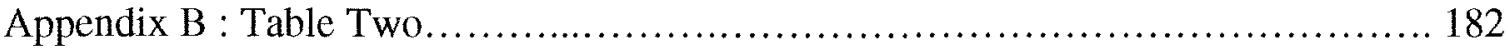

Appendix C: Tables Three and Four........................................... 183

Appendix D : List of Stories in The Sample................................. 184 


\section{CHAPTER I}

\section{Introduction}

On April $20^{\text {th }}, 2010$, BP's Deepwater Horizon oil platform exploded in the Gulf of Mexico. It sank two days later, leading to a massive oil spill that originated miles below the surface and bled into the Gulf for months. While watching news coverage of the ongoing spill, I was struck by the critical tone of the coverage; many reports focused on the failures of CEO Tony Hayward and BP. Although the spill would become a transnational problem, spreading through the water across imaginary political boundaries, media coverage appeared to focus narrowly on the liability of foreign-owned BP, while ignoring the network of corporations and regulatory bodies-mostly American-who were complicit in the conditions leading to the spill.

Similar to many environmental disasters that preceded it, the Gulf spill was neither an unforeseeable accident nor the consequence of a few bad judgments made by a handful of bad people in charge of mega-corporations and federal oversight bodies. In the short-term, the spill can certainly be blamed on poor judgment, technological failures and a lack of regulatory oversight, as well as a failure to prioritize safety. However, all of these factors are intimately linked to economic changes that began in the 1970's with the rise of neoliberal economic ideology, which has slowly come to dominate the policies of transnational corporations and governments worldwide.

Molotch and Lester (1975) conducted a study on the media responses to the Santa Barbara oil spill of 1969. They discovered that unexpected events like oil spills may temporarily challenge the conventional organization of media access. Oil spills are unplanned events and even major sources are unable to prepare media responses. Thus, 
they determined that challengers to the status quo may have higher than usual access to media following an oil spill, but that this access quickly fades. They explained that sources such as the President or executives of large corporations are always considered to be more newsworthy than groups like conservationists, even when these powerful sources are repeating routine news items. Molotch and Lester (1975) also suggested that the local economy plays a strong role in the portrayal of industrial environmental accidents. With these findings in mind, my study also looks at print news responses to a sub-sea oil leak and bears much in common with their study. There are many intriguing parallels between Molotch and Lester's (1975) study and my own, but there are also several important points of departure. Epistemologically, I depart from Molotch and Lester (1975) who argue from a stance that there is no objective reality to be known. Although I share their view that objectivity is impossible to achieve in media, and news stories are of course constructed, my project is rooted in studies of political economy and the material realities on which they are based. Thus, I often suggest that the political forces and the economy have concrete effects on institutions, people, and systems of meaning.

My study looks at a similar event to the one studied by Molotch and Lester (1975), but over 40 years later and in a different area of coastal United States. As will be explained shortly, there have been powerful economic changes in the American and global economies and political cultures in these 40 years, prominently the rise of neoliberal thought and practices. As well, the economies and politics of Gulf States are much more influenced by large oil corporations than costal California. Finally, I will be examining a media world that has changed dramatically due to new technologies and organizational practices in the last 40 years. 


\section{Research Questions and Argument}

The news coverage that appeared in the wake of the Deepwater Horizon disaster has led me to ask, how are environmental accidents framed in news media? Does that framing process echo trends in the broader political economic climate, and if so, how? My research process is exploratory and began with questions about how environmental incidents are portrayed in print news. After conducting a wide scan of my research material and a detailed conceptual coding process, I detected clear resonances between neoliberal thought and practices and the information presented in the media sample.

My thesis explores the relationship between how media framed the spill and patterns of reliance on particular news sources. I employ frame analysis to examine the content of seventy New York Times online articles, which were selected at key intervals in the seven months following the spill. Broadly, my approach involves examining whether and how sampled news coverage either challenges or incorporates the viewpoints and frames of major sources. I look for common narratives within the prominent source frames from various sources, as well as examine how these sources have been constructed in the news. I carefully consider varying levels of access among prominent groups that have a vested interest in the public understanding of the spill. These groups include BP, the US government, environmentalists, and residents of the Gulf coast. I consider this access in terms of a hierarchy of trusted and authoritative sources.

My exploration began with the hypothesis that there would be little discussion in the mainstream print media of the economic and political causes of the Deepwater Horizon rig explosion and subsequent oil leak. Although this held true, after engaging in 
an iterative relationship with the literature and data, my argument subsequently evolved to suggest that the frames present in the spill coverage evidenced a neoliberal ideology in a number of ways. This argument is rooted in the tendencies I found for news coverage to individualize social problems; minimize the portrayal of structural causes and repercussions; depoliticize and trivialize local voices; and prioritize corporate interests and the economy over the environment and local people. Thus, although the content of my sample is wide and diverse, it generally promotes the continuation of the status quo that led to the spill. Neoliberal ideology was especially prominent within pro-business, $\mathrm{BP}$ and oil industry frames.

There has been a great deal of research into the political economy of news production, much of which argues that the media reproduces elite discourses, generally due to corporate ownership (McChesney, Herman and Chomsky). Although my argument could easily be situated in this literature alone, I problematize the somewhat deterministic nature of this literature by incorporating the sociology of news production and media studies. Those who study the sociology of news often argue that journalists are relatively autonomous. Rather than deliberately acting as a mouthpiece for corporate and government interests, they are instead constrained by the organizational routine of news production in ways which have the same ultimate outcomes in terms of the ideological tendencies of the news (Anderson 2002; Schudson 1989; Molotch and Lester 1975).

These two perspectives tend to converge on one important point: resultant news reports often individualize broader social problems, rely heavily on official sources for news, avoid systemic discussion of long-term economic and political policy, and omit alternate sources. I contribute to this literature by examining the frames present in the 
news coverage of a contemporary environmental industrial accident. My contribution also includes a synthesis of these literatures. I detect the initial framing packages using the top-down approach of the political economy perspective, but then orient these frames within the constraints faced by members of the media when creating news.

\section{The Macondo Blowout of 2010: Background and Context}

On the fortieth anniversary of Earth Day, the Deepwater Horizon drilling rig crumpled into the Gulf of Mexico and began its long journey to the seabed, over 1.5 kilometres below. Unlike older well-drilling rigs, the Deepwater Horizon was not attached to the seafloor. Instead, it drilled while semi-submerged toward the Macondo reservoir five kilometres below the ocean floor. When the blowout occurred, oil and gas began to leak from the damaged wellhead, although this was not formally acknowledged until more than a week later. BP attempted a number of failed efforts to contain the oil. The first, a 98-tonne containment cap, failed on May 8, just one day after it was lowered. BP tried again in late May with "top kill," a new procedure that would force feed the well drilling mud. Fresh on the heels of President Barack Obama's controversial ban on new deepwater drilling projects, the top kill was abandoned on May 29. For the better part of June, the media focused on oil-soaked beaches, conflicting views on the spill rate, and President Obama's alleged failure to lead during the disaster.

On July 27, BP officially announced a plan to replace their CEO Tony Hayward with Robert Dudley, an American raised in Mississippi. The flow of oil, however, was not significantly reduced until August 4, with the "static kill." Soon after, scientists and media learned that the spill rate had been much higher than either BP or the US government suggested. On September 8, BP released the results of their internal 
investigation, in which the company claims it had not acted negligently. On September 19, after completion of a relief well, coast guard admiral Thad Allen deemed the Macondo well 'effectively dead.' Although it is almost guaranteed never to leak again, the drilled cavern to the oil reservoir is now a permanent feature of the seafloor. In January, the Presidential Commission finalized their report, which suggested there had been numerous errors leading up to the spill.

$\mathrm{BP}$ and the US federal government consistently under-estimated the rate of oil flow. Initial reports stated that there was no leakage whatsoever. After evidence to the contrary became irrefutable, both parties suggested that 1000 barrels a day could be escaping from Macondo. Internal BP documents, however, stated that the well could leak up to 150,000 barrels a day. A short time later, the official estimate was upped to 5000 barrels, and by August, there was a general consensus that the spill began at 62000 barrels per day. By the time it was capped, the leak had only slowed to 53,000 barrels per day (Freudenburg and Gramling 2011). At this rate, the Gulf spill eclipsed the Exxon Valdez disaster in under five days.

Throughout most of June and July, a four-inch pipe siphoned oil from a 21-inch leak to a tanker on the surface. Between oil collection, skimming and burning, BP managed to 'recover' just 900 barrels per day. After months of containment, the company succeeded in eliminating a total of just over 300000 barrels. However, their recovery plan indicated a capacity to remove 491,721 barrels per day (Freudenburg and Gramling 2011). The Macondo blowout is now the largest accidental marine oil spill on record. The only spill to eclipse it occurred in Kuwait, when spigots were deliberately opened and ignited during the Gulf War. Other sources suggest that decades of oil activity in Nigeria 
have led to a cumulative spill volume equal to or greater than the BP spill (Sokari 2010; Vidal 2010). However, a complete absence of oversight or regulation means that the total spill volume in the Niger delta's 606 wells remains highly contested (Vidal 2010).

BP operated and leased the Deepwater Horizon drilling platform for half a million dollars per day from a company called Transocean, which owned and built the platform. During construction, Transocean contracted out to companies including Halliburton, which completed the final cementing job on the well. As outlined in the analysis, much of the discussion of culpability involves BP blaming Transocean and Halliburton, while those companies deflect blame back toward BP. The US government and President Barack Obama were the focus of intense criticism after the spill. Clean-up was largely the responsibility of the Coast Guard, but offshore drilling industry oversight had been the responsibility of the Minerals Management Service. After the Gulf spill, MMS became the Bureau of Ocean Energy Management, Regulation and Enforcement.

Many expert voices and alternative media sources have begun to highlight ongoing spill-related health issues and a declining quality of life on the Gulf of Mexico (Whitty 2010; Safina 2011). While the spill was still receiving intense media coverage, many senators touted their commitment to increased legislation and oversight, but as of this writing there have been no new successful legislation or rules (Safina 2011; Freudenburg and Gramling 2011). When Deepwater Horizon exploded, there was a $\$ 75$ million liability cap on oil spills for corporations that were not found to be criminally negligent. Many government officials called for this cap to be raised, but this has yet to be acted on. BP has not evoked their right to use this cap, and instead volunteered a 20 billion dollar compensation fund. Some commentators have suggested that BP only offered the fund to 
pre-empt government instructions (Mene 2011). Approximately half of the fund has been paid out, and many Gulf coast residents have suggested the claims process is needlessly complicated and slow (Mene 2011).

The physical site of the spill is environmentally significant and hosts many delicate ecosystems. In early 2010, the decade-long Census of Marine Life ranked the Gulf of Mexico as the fifth most diverse ocean habitat on Earth (Freudenburg and Gramling 2011). The Mississippi Canyon, where Deepwater Horizon operated, is within miles of a huge current that leads to the Gulf Stream and spreads throughout the Atlantic Ocean. As well, the Gulf is underneath one of the most important migratory bird routes on Earth, and borders almost fifty percent of America's remaining wetlands. The Gulf spill began in the spring and continued into summer, contaminating an entire spawning and hatching season for many animals (Freudenburg and Gramling 2011). Many scientists featured in alternative media have suggested that the effects of the spill have only just begun and may resonate in the food chain for decades (Freudenburg and Gramling 2011; Whitty 2010).

\section{Political Context}

The Deepwater Horizon explosion and subsequent spill is a catastrophe that can partially be attributed to the neoliberal trend in economic policy over the last twenty years (Kirsch 2010; Harvey 2007). As I will explain in more detail in Chapter 2, neoliberal economics calls for reduced regulation, smaller government, and a deep-seated trust in the ability of the free market to gradually guide corporations to the best solutions to humanity's problems. A core component of neoliberal economic ideology prioritizes the free market as a value unto itself. This free market ideal supersedes any other value 
system, including any that prioritize the health of the planet (Harvey 2007, Treanor 2005). Continuous economic growth and expansion is also a core value; proponents of neoliberalism argue that corporations should be granted the freedom to self-regulate. In the US, corporations are charter-bound to prioritize their own profit margin. In an increasingly lax regulatory environment, this is implicit permission to consume natural resources and produce large amounts of waste. The values of neoliberalism are fundamentally antithetical to those of environmentalism.

Despite the wider social responsibility for this disaster, a starting point for this thesis is that media interrogation has remained primarily at the individual level. Immediately following the Gulf spill, the US news media ran almost nonstop coverage condemning BP and CEO Tony Hayward. This focus is consistent with previous research demonstrating that media coverage of the huge Exxon Valdez spill in 1987 focused on the ship's drunken captain, while paying little attention to many of the broader issues at stake (Anderson 2002). This tendency to reduce complicated events to individual failings has been observed by a number of media scholars (Anderson 2002; Molotch and Lester 1975) who have likewise noticed a lack of discussion on structural issues, i.e., the converging interests of regulators, politicians, and corporations.

As the oil and dispersant drifted into international waters, this crisis began to physically transcend national boundaries. Yet from the moment the platform exploded, the web of responsibility extended to corporations, free market policies, failed regulatory structures and a government heavily influenced by corporate lobbyists. Eroded regulations and depleted oil reserves have sent oil companies exploring at greater depths without appropriate technology or clean-up capabilities (Whitty 2010; Freudenburg and 
Gramling 2011). Without improvements to the regulatory environment, corporate practices, and economic policies, environmental industrial accidents are likely to increase in frequency.

\section{Cultural Significance}

The BP oil spill is an ideal site for sociological mass media investigation. The oil spill became one of the most publicized stories of 2010 , a key political topic, and the subject of investigations by BP, Transocean, and the US government. The Exxon Valdez spill became a key symbolic event in the public's psyche, representing the tension between economic progress and environmental protection. This decade, the Gulf oil spill may take on similar environmental and social significance. Although oil has stopped flowing from the Macondo well, the consequences may not be fully understood for many years to come.

$\mathrm{BP}$ recently underwent a massive effort to brand the company as green. In the year 2000, British Petroleum became Beyond Petroleum, complete with a new green logo (Kirsch 2010). This significant marketing effort makes BP's environmental transgressions an ideal case for investigation and scrutiny. This re-branding strategy reveals an exceptional interest in the burgeoning green energy sector, while the circumstances around the spill reveal that this was predominantly a public relations effort. After the Gulf spill, BP became a model for both green marketing efforts and a contemporary environmental catastrophe.

Many Americans witnessed the coastal damage unfold in Alaska after the 1989

Exxon Valdez spill, and some also witnessed the Californian Santa Barbara spill of 1969. Although it is less often discussed, Ixtoc I suffered a blowout in the Gulf's Mexican 
waters in 1979. Until the BP spill in 2010, Ixtoc I was the largest accidental marine oil spill in history; it even polluted the Texas coast. But because the BP oil spill was the first major oil spill in North America in more than 20 years, it may resonate more strongly with younger Americans. For over a month, viewers were able to watch the Macondo pipe leak into the water in real time. They witnessed multiple attempts to plug the well fail, though even without an engineering degree, the problem seemed simple enough.

Exxon disaster had a devastating impact on wildlife and beaches, but it was nonetheless isolated in Alaska. On the other hand, the BP spill had clear physical consequences for five mainland states. Coverage of the Exxon spill did include dramatic imagery of oiled animals, but the tanker had run aground and leaked its contents almost immediately. There was intense public pressure on Exxon to clean up the toxic beaches, but media coverage of the event did not feature the same level of suspense as the Gulf disaster. Interested viewers and readers waited time and again to see if engineers would succeed using one plugging technology after another. Meanwhile, much-disputed spill volume estimates continued to rise. Viewers and readers watched BP fail repeatedly while CNN ran constant live footage of the damaged pipe spewing oil and gas into a defenceless sea. For these reasons, this disaster could emerge as a key event on the American environmental compass.

\section{Thesis Organization}

This project is divided into six chapters. Following the introductory chapter, there is a review of the substantive and theoretical literature, which sets the political and economic context in which the spill occurred. The chapter also provides a detailed account of neoliberalism and its relation to the data that follows, as well as an overview 
of media theory, environmental sociology and sociology of media. In Chapter Three, I provide a detailed overview of the methodology and research process, including the rationale for my sampling procedure and choice to conduct a frame analysis. The data analysis is presented in Chapters 4 and 5. Chapter 4 focuses on voice and access: who is quoted in media reports and how reporters choose to quote them. It explains the significance of access and discusses the content of access in a more qualitative fashion. Chapter 5 extends the conceptual reach of the analysis by looking at how media reports are framed and which themes are highlighted. These chapters together provide answers to my research questions by exploring the spectrum of voices and frames that are represented in the wake of a highly publicized environmental industrial accent, and connect these research questions to a cultural and economic climate of increasing neoliberal ideology.

\section{Contribution to the Literature}

There exists a rich literature on the effects of neoliberalism, corporate influence in government, concentration of media ownership, the continuous evolution of media, and the rise of environmentalism in media and public discourse. The specific aim of my project is to mobilize these research areas with reference to a contemporary environmental disaster. This research will contribute to academic and public awareness of how government, media and corporations react to ecological crises, and evaluate the discursive landscape created through disaster response. Previous research in political economy and sociology of media has found that news focuses on event details and privileges official sources, while neglecting in-depth critique. However, there are 
discrepancies and debates within this broad body of literature on explanations for the media's tendency to avoid analysis.

My thesis builds on this research by applying literature on political economy of media and sociology of news production to a recent, highly publicized environmental incident. Due to technological, economic and cultural shifts, media are evolving rapidly (Carlson 2009). Research into the contemporary media climate is necessary in order to understand how these changes are unfolding and how the media should perform in order to best inform the public about events. Evaluating how the New York Times is able to construct and give space to certain sources and voices reveals the spectrum of values present in newsrooms while also shedding light on the corporate and government responses to an industrial incident.

Although certain print media outlets have included discussion of the economic conditions that led to the 2010 leak (Whitty 2010), I have been unable to locate an academic study of media reactions to the spill. Likewise, I have yet to find a study that specifically examines the role of neoliberal discourses in the spill response. The Deepwater Horizon is the largest oil spill in US history. Due to the generous use of toxic dispersant in clean-up, the spill may present a serious threat to international waters and marine ecosystems for many years (Robertson and Krauss 2010; Whitty 2010).

Environmental news coverage requires that members of the media make sense of technical and scientific information, while evaluating the high volume responses from BP, government, locals, environmentalists and other interest groups. Each of these narratives is structured into an ongoing event, which must be carefully integrated into 
daily news (Anderson 2002). Thus, the contested space of media on this crucial environmental and social event is a key site for research attention.

The nature of this project as a media study also requires that I contribute to the ongoing debate regarding the level of influence that concentrated corporate ownership has on the spectrum of information available in the media. Although the scope of this project does not allow me to interview members of BP or environmentalist organizations, or to survey the public on their absorption and interpretation of the news, the content of the news is important to study for its own sake. This project enables me to discuss one of the largest oil companies on the planet, in the context of the largest oil spill in US history, which has produced what is likely to be the highest volume of media coverage since Exxon Valdez. I will use the oil spill to explore the way media constructs reactions to environmental accidents and extrapolate that understanding to look at the social and political importance of media more generally. 


\section{CHAPTER II}

\section{Theoretical Context: Sociology of News Media and Political Economy}

My thesis project began with a broad investigation into the news media response to the 2010 Gulf oil spill. In conducting this project, I ask: how are environmental accidents framed in news media; does that framing process echo trends in the broader political economic climate, and if so, how? By engaging with the literature in this chapter, I demonstrate that the construction of disasters in the news media is a rich area for sociological investigation, particularly in a time of increasing neoliberal influence on governments and businesses. A key interest of this study is to evaluate whether alternate voices can effectively access openings in the media landscape during an environmental accident, or if priority is given to authoritative sources like governments and scientists.

The protections and regulations of many national governments across the globe have been gradually eroded while multinational corporations continue to expand. This trend is especially pronounced in the United States, the site of the spill. The chapter begins by exploring this political economic context, thereby explaining the theoretical significance of neoliberalism for this project. I outline several specific discursive themes within neoliberal ideology in the literature; these are particularly relevant to media analysis. Neoliberal discourse focuses on individuals to the neglect of systemic issues and often focuses blame narrowly on particular deviant persons. This rhetoric generally prioritizes business solutions and suggests that the market will guide society through crisis, while also minimizing the potential harms that have arisen from this economic policy. Neoliberal discourses are also known to omit other structural explanations for social problems, including environmental incidents. 
I draw on literatures that analyze the news media environment. I will discuss different perspectives in the sociology of news production. The sociology of news explains trends in media ownership, news values, sources, and the objectivity paradigm, all of which constrain what journalists produce. I give special attention to news coverage of environmental issues and oil spills. Finally, I discuss my use of the terms discourse, ideology and narrative, and the theoretical implications of my working definitions. These terms are discussed in relation to frames and framing - the broad theoretical packages that are later extracted from the data. This chapter provides sociological and political economic contexts for the media investigation to follow. The review demonstrates that I have chosen a valid topic for investigation; there are avenues for contribution within literature on the changing media landscape, as well as on mass media response to environmental disasters.

\subsection{Political Economy and Neoliberalism}

More than fifty years ago, C. Wright Mills (1956) prophetically identified a narrowing gap of interest between the dominant spheres of power in the western world. He argued that political, economic and military elites were converging and power was becoming more concentrated in the hands of fewer men. ${ }^{1}$ These elites shared similar interests that were later articulated through the shared values of neoliberal economicsan ideology that suggests that the greater good can be best achieved through free markets, free trade and private property rights. Harvey (2007) details how neoliberalism has permeated the common sense of news media and people worldwide, popularizing the

\footnotetext{
${ }^{1}$ Mills only referred to men, as in 1956 almost no women held any positions of government, business or military authority.
} 
view that the role of the state is to protect trade and private property, and defer to the wisdom of the free market. Harvey (2007) explains that neoliberalism is a diffuse and increasingly global economic philosophy promoted by elites to increase their wealth and power through free trade and small government. He suggests that neoliberalism has not only become the dominant economic policy in the United States, but is also increasingly prevalent worldwide.

A crucial component of neoliberalism is that it is more than 'new liberalism' or a revival of the free market. Neoliberalism is a philosophy derived from classical economic liberalism, which teaches that the existence of the free market is the only worthwhile end, and that the role of the state is to protect markets and wealth. Classic liberalism is theoretically lacking in any values beyond the self-determination of the market. In a neoliberal utopia, the state would only interfere with corporations insofar as that interference ensures the continued existence of the market-a security protocol that protects the continuation of extreme wealth inequity (Treanor 2005). Neoliberalism is a social and moral philosophy that values the existence and operation of the market above all other human needs. While classical liberalism suggested that society should be determined through the market, neoliberalism suggests that the market $i$ s society, and that theoretically, it should financialize all elements of social life. Neoliberalism is promoted through the elevation of freedom and cultivates the assumption of an intimate connection between personal freedoms and market freedoms (Harvey 2007). Although this is a global trend, the United States especially tends to characterize the market as a crucial part of national identity. 
An essential component of my analysis hinges on the notion that neoliberalism frames the world in economic terms (Fairclough 2000: Hallin 2008). Neoliberalism presents life as a series of market transactions, and does not acknowledge the complex stratum of social, environmental, cultural and economic factors which influence quality of life. This tendency explains the neoliberal use of market metaphors to discuss elements of social life that have nothing to do with buying or selling (Treanor 2005). Harvey (2007) explains that if 'true' neoliberalism were embraced, no element of our social lives would be divorced from market competition, and there would be no focus on any system other than the economy. Thus, neoliberalism is fundamentally at odds with environmentalism, as well as any other value system that measures merit beyond the market. Environmentalists consider the natural world to be inherently worth protecting, much as neoliberals view the market. Economic policy that prioritizes short-term wealth and limitless profit margins has had devastating effects on the worlds' ecosystems, from global warming, deforestation, and the promotion of a carbon-heavy, consumption-driven lifestyle. The tendency to reduce social life to a market transaction is explored in the data analysis.

Neoliberalism also individualizes social problems by focusing on the individual, who tends to be described as either problematic or successful. This tendency is derived from the idea that the state should not provide social protections, instead leaving this responsibility to individuals who are each held fully accountable for their success or failure (Harvey, 2007). Neoliberalism responsibilizes deviant people and institutions, and blames them for the problems they face. This policy has the combined effect of justifying the security state as a protection against criminals, normalizing the failure of presumably 
flawed people, and reducing social problems to unlikely accidents. Likewise, neoliberal economic policy marginalizes structural narratives beyond the advancement of business. Neoliberalism may promote systemic solutions with the caveat that a 'system' includes smaller government and advancement of the market. There is an intimate link between neoliberalism's tendency to individualize social problems, promote free market solutions and omit alternate narratives.

\section{$\underline{\text { 2.2 Media }}$}

At its core, this thesis is an investigation of media. The media are of interest to sociological inquiry because they are the primary communicative link between people and their institutions. Likewise, mainstream news helps set the political agenda and frames the terms of public debates (Scheufele and Tewksbury 2007). Countless researchers have noted that mass communication exists as a realm of inquiry because exposure to media has substantial effects on public perception (McQuail 1994; Bell 1995). Media analysis allows the researcher to explore the information relayed to the public and the efforts of major institutions to access the media.

\section{Political Economy of Media and Ownership}

Many media critics suggest that mainstream news coverage promotes neoliberalism and the economic status quo because major news outlets are themselves multinational corporations owned by business elites who exert powerful control over the products of media (McChesney 2001; Herman and Chomsky 2002). McChesney (2001) studies the political economy of media and argues that because of its corporate ownership and structure, the production of news is an industry that is inherently biased to benefit the interests of owners of capital and maintain the economic status quo. Likewise, Herman 
and Chomsky (2002) argue that the news indirectly promotes the agenda of the economic elite through the views preferred by hiring committees and the news values that journalists are instructed to uphold. Herman and Chomsky (2002) understand the media through a "propaganda model" which indicates that though the media performs a variety of functions, they primarily disseminate information in favour of the corporate elites that own and finance major news sources. Herman and Chomsky's propaganda model explains media behaviour through five filters, including concentrated corporate ownership and reliance on expert sources. The authors are careful to explain that the forces that influence media do not need to be direct, or all controlling, nor do they produce a seamless and identical result in every case. They go on to explain that though the media disseminates propaganda, the audience does not always receive or accept it. Nonetheless, this view suggests the media debate leaves little room for contestation or resistance to institutional power (Schudson 1989).

This thesis aims to engage the above literature by discussing the extent to which a pro-corporate discourse is reproduced in my media sample. Neoliberalism provides an ideological rationale for transferring media to private transnational institutions; functioning according to this rationale, media businesses have reduced their budgets, thus reducing their capacity for investigative journalism (Kimball 1994). Due in part to budgetary and time constraints, media responses to an environmental event may focus on the event itself rather than analysis of its causes or long-term repercussions. Rather than creating direct interference, neoliberal economics may be considered indirectly to blame for the media's unwillingness to discuss structural issues.

\section{Constraints on the News Media}


Alternate accounts of the media's failure to report structural issues are oriented less toward corporate ownership. and focused instead on structural constraints within the media. Anderson (2002) explains that the media tends to focus on guilty or responsible parties rather than structural issues. This tendency is caused by many factors, including the 24-hour news cycle, event orientation, and a reliance on expert sources. Anderson (2002) cautions researchers against the presumption that news coverage simply manufactures public opinion. My efforts to respond to my research questions require investigation into the extent to which news media reproduces a focus on individuals and an event orientation, and reinforces the legitimacy of expert sources.

\section{Sources}

Anderson (2002) suggests that media discourse on oil spills demonstrates an overreliance on official or expert sources. Molotch and Lester (1975) consider the media's routine and institutionalized over-reliance on official sources to be a key reason the media fails to challenge conventional economic wisdom. This observation is echoed by a number of media analysts (Hall et al 1978; Gans 1979; Bennett, Lawrence and Livingston 2007). Carlson (2009) confirms that one of the most common observations in all media studies is a reliance on official sources. Official sources are often large and bureaucratically organized, i.e., state and federal government, think tanks, special interest groups, and relevant corporations. The individuals who predominantly represent these sources are white men (Brown et al. 1987). Men still dramatically outnumber women in political office and other official positions, including corporate board membership. By privileging these authoritative voices, the media simultaneously excludes women from 
media coverage and reproduces the view that men are authorized knowers who are entitled to their authority.

Carlson (2009) explains that there are competing viewpoints on the relationship between journalists and their sources. This relationship is characterized as either mutually helpful (with sources controlling the discourse), or as competitive (with sources and journalists competing over their ability to control the news). The analysis in Chapters 4 and 5 considers a contemporary environmental disaster in relation to source/journalist relationships by revealing quotation patterns and the content of quoted sources.

The media often reproduces dominant discourses, particularly through their sources, many of which tend to have a vested interest in maintaining the status quo (Herman and Chomsky 2002). Carlson (2009) confirms that by their nature, trusted sources have the power to present and reinforce their version of society. By being used as sources, these authoritative figures are granted further legitimacy. Examining media sources provides a valuable path of sociological inquiry into the construction of power in public discourse. Schudson (1979) suggests that the media often rely on official sources because these sources and media outlets are all bureaucratically organized and easy to access through official channels. The tendency to rely on official channels for sourcing reflects the constraints placed on journalists through the reality of their work lives and the time constraints they face.

Becker's (1967) classic account of media sources arranges them in a hierarchy of credibility. He explains that sources are ranked; the higher your ranking, the more authority and power you have to control reality. This hierarchy is useful in that it reinforces the idea that the spectrum of potential sources for a news story is not 
assembled in an open market of ideas. However, this hierarchy may not necessarily prove useful in assembling an overview of the reactions to a sudden environmental event, such as an oil spill. When an event is dynamic and unforeseen, sources may not be arranged as neatly from most to least credible. A guilty corporate party may rank much lower on the hierarchy because their motives are suspect. However, a corporation may still receive ample opportunity to present their frame. Affected local residents may not be considered credible, but may nonetheless be integrated for the sake of objectivity and balance.

Becker's (1967) hierarchy of expert sources introduces the notion that sourcing patterns reflect broader dynamics of power. That is, some groups' voices are granted more authority than others. Although a sudden an unexpected event like an oil spill complicates this hierarchy, Becker's design sets the stage for power struggles between sources. Molotch and Lester (1975) begin from a conflict-oriented approach to news, as evidenced through sources competing to define public events. Other scholars also suggest that studies of media all investigate and reveal of imprints of power (Entman 2002). This power lies in the capacity to create and design public events (Molotch and Lester 1975). Thus, studies of media access evaluate public construction of power relationships. As discussed above, there are debates in the literature over whether journalists or sources are wielding the most power (Carlson 2009). Studies of the media track how competing actors fight over this shifting imprint of power, and how the public event in question can shape this dynamic while contests within the dynamic simultaneously shape the event.

Media play the dual role of informing the public about policy issues and acting as a watchdog that challenges the way these issues are presented to the public (Callahan and Schnell 2001). Media are responsible for informing the public of transgressions by 
institutions such as the state and large businesses. As this chapter presents, there is an ongoing debate as to what extent media succeeds at-or even attempts to-critique dominant institutions. Carlson (2009) suggests that the media's routine reliance on elite sources allows these voices to set the frame of a story; alternate voices are expected to react to this frame, and are rarely invited to generate their own. Though alternate framers may be included in the debate, they are not necessarily given an opportunity to set the tone. The organization of sources in media highlights the importance of studying who is quoted first in an article; those sources may have greater access to the broader framing package. In Chapter 4, I evaluate the tendency for media to disproportionately quote the voices of governments and corporations, while giving comparatively little access to affected local residents or other interest groups. One focus of my thesis is to examine and weigh sourcing patterns and to connect these patterns to the reproduction of power or resistance by alternate sources.

Event orientation and the 24-hour news cycle

News coverage focuses on distinct dramatic events in the immediate presentknown as "event orientation"- to the neglect of a more complex, historically contextualized, political and social analysis (Ericson, Baranek and Chan 1987). This tendency is linked to a large number of possible causes, including the perceived appetites of the audience, concern with sales, unwillingness to commit time and money to investigative journalism, and nigh-immediate knowledge of global events. A major contributor to this trend is the 24-hour news cycle. This term refers to the structure of a newspaper. A newspaper is considered a wrap-up of the day's events, so it tends to focus on events that happened in the previous 24 hour. Oil spills are long-term events that 
media often needs to divide into manageable daily stories even if this style of organization betrays the nature of the event. Combined, these tendencies contribute to a focus on individual problems that can easily be explained within the confines of the format. Anderson (2002) examined news coverage of the huge Exxon Valdez oil spill and explains that media predominantly focused on the drunken captain, despite the presence of broader changes in the economy that were pushing tankers to work at lower cost. Anderson (2002) explains that news coverage is based on events rather than issues, which is echoed by several other media scholars (Hansen 1991; Molotch and Lester 1975). It is in the interest of oil companies and the government to place the blame on a particular employee or on a series of human errors; this procedure is preferable to an indictment of the company, industry or economy as a whole. News media may frame a story as a manageable event rather than a complicated long-term social problem because of the demands of the 24-hour news cycle.

Objectivity Paradigm

Most media scholars agree that Western journalism is guided by the objectivity paradigm (Schudson 1989; Carlson 2009). Journalists have often been trained to remove their opinions from the work; the use of sources is thus closely linked to an appearance of objectivity. By frequently citing a source, the bias in a report can be attributed to that source rather than the journalist. Sources provide crucial information for a story, while also lending it legitimacy (Carlson 2009); the more 'sides' of a story a journalist presents, the more unbiased that story appears. Carlson (2009) explains that by trusting sources, the quoted source material is often accepted as fact, while the journalist's view is 
considered suspect, particularly when it appears in event-oriented and breaking news pieces.

There is a general consensus among the discussed media scholars that the news is constructed and created by journalists and their sources, Molotch and Lester (1975) ardently dismiss the notion that journalists can ever be objective because, as they suggest, there is no objective reality to be known. Molotch and Lester instead ask how a given reality is privileged in the news. The practices of journalists and the news they create are thus of key sociological interest since they produce a powerful version of events and experiences as reality.

That media strive to be objective is a powerful notion. Gitlin (2003) explains that this presumption infuses media with the power to significantly alter public perception on the legitimacy of different voices, and create a broader understanding of what common sense actually entails. Schudson (1989) cites the political economy approach to media analysis, which acknowledges that a commitment to objectivity in newsrooms somehow leads to news that seldom challenges the state or corporations, and instead reaffirms the status quo. He argues that the ideological power of news is the widespread belief that this material is objective.

\section{Media, Environment and News Values}

There is a rich body of literature that deals specifically with media coverage of oil spills. Anderson explains that members of the media who report on environmental issues are faced with the task of summarizing and simplifying complex scientific information, which inevitably leads to some distortion. Although this is a common problem faced by journalists reporting on any scientific issue, it is especially prominent in environmental 
science. When compared to health or other streams of biology, environmental science is relatively new to mainstream discourse. Despite the ascent of neoliberalism, some scholars also argue for the existence of countervailing tendencies. That is, the mass audience has fragmented and the media market has become more heterogeneous. More news outlets now target specific audiences, potentially creating more openings for a challenge of the status quo. Simultaneously, the influence of the environmental movement has grown (Anderson 2002).

In their classic media study, Molotch and Lester (1975) examined media coverage of the 1969 Santa Barbara oil spill in order to explore power relations among government, corporations, media and environmentalists. They found that pro-drilling voices (government, oil companies) had greater access to the media than did environmentalists or local residents, although major environmental accidents could create temporary openings in the media for voices that challenged the status quo. They suggested that these openings were created because of the nature of an oil spill: it is as accidental news. Powerful corporate and government interests would never want an oil spill to be in the news in the first place. While the government would attempt to control the initial framing of a declaration of war or choice to conduct deepwater drilling, there is little planning or routine response to a sudden event. Thus, the sudden event disrupts routine news coverage; alternate framers (conservationists, locals) may be able to take advantage of this space in the news. However, Molotch and Lester specified that this tendency was related directly to the geographic proximity to the spill site and the political economic context of the site itself. Their study also took place in the 1970 s, before neoliberal economics became commonplace in many governments and corporations. 
Thus, it is important to reassess these findings and evaluate the extent to which sudden events like an oil spill continue to create openings for challengers to the status quo. It is also important to evaluate the representation of major actors such as government, oil corporations, conservationists and locals.

News media are a main artery to public knowledge on environmental issues. Hannigan (2006) echoes other literature that describes many of the aforementioned characteristics of news, including its reliance on expert sources, event orientation and adherence to organizational routine. He suggests that news is the primary means by which citizens are educated about environmental issues. He reminds the reader that the creation of news is part of a system of work; it strives to bring order and readability to the chaotic series of events that occur in the run of a day. With these institutional constraints in mind, Hannigan (2006) criticizes the media for failing to ask 'why,' and thus failing to situate environmental events as a part of economic, political and social history. This is a useful opening within which I place my research, which also evaluates the extent to which the members of the media and their sources ask 'why,' and how this question is answered. The tendency to omit the 'how' and 'why' from media discourse may cause readers to blame particular companies or individuals rather than seeing the link between environmental degradation and broader structural problems (Hannigan 2006).

This type of reporting also fosters the impression that when individuals and corporations fail, blame can exclusively be placed on their shoulders. This approach fails to consider that issues are intimately connected to corporate norms and economic macrostructures. Hannigan notes that although environmentalism in media coverage is relatively new, and stories continually increase in number, gravity and global focus, they 
do not necessarily promote a deeper understanding of environmental issues. He explains "by focusing on discrete events rather than on the contexts in which they occur. the media tend to give news consumers the impression that individuals or errant corporations rather than institutional politics and social developments are responsible for these events" (Hannigan 2006:126). The event orientation of the news allows journalists to focus on the details of a particular environmental occurrence, while neglecting a structural critique of environmental practices.

Anderson (2002) looks at how the unique features of an oil spill affect journalists perceived newsworthiness of the event. She highlights the reasons that an oil spill is considered particularly newsworthy through examination of news values. which represent deeply entrenched organizational conventions. She expands upon the oft-cited eight news values that Galtung and Ruge developed in 1965:

- Frequency - the news media generally function on a 24-hr cycle; gradual environmental problems may be ignored if there are no new developments.

- Amplitude - an event must reach a certain size before being considered worthy of reporting; the larger it is, the more worthy of coverage.

- Ambiguity - the clearer an issue may appear to readers, the more worthy it is coverage; the complexity of environmental issues may affect the level of coverage they receive.

- Meaningfulness - media often focus on stories that are closer to home, familiar, and relatable to readers' daily lives. This is reflected in Molotch and Lester's comparison of local and national news in the Santa Barbara spill (1975).

- Consonance - stories are often built on pre-existing conventions for that type of story; a familiar stereotype, frame or narrative will often be reproduced. 
- Unexpectedness - the more surprising an event, the more worthy it is of coverage. For example, not only is climate change old news, but also more challenging than an oil spill to fit into the day's reports.

- Continuity - an item that has already been covered is still considered newsworthy, even when scale is diminished; follow-up on an oil spill story is considered relevant, even when there is little new information.

- Composition - news is designed to reflect certain topic areas, as reflected in the organization of newspaper sections. Thus, some stories may receive attention in order to fill an empty section.

According to the above criteria, a large oil spill would be generally considered quite newsworthy. Spills are often dramatic, immediate and unexpected by the general public. They may pose a pronounced threat to the everyday lives of those in coastal communities, or those who work in the fisheries. Oil spills can easily follow a journalistic convention, since they have happened many times in the past. Although there is often a complicated set of political conditions that lead to such an event, they can often be reduced to a simple narrative. Spills are ongoing events until plugged, capped, or cleaned up, and thus generally receive news updates even if there have been few developments.

Although these values are a useful reference, they are not static criteria by which to judge the coverage of an event. Anderson (2002) explains that when reporting on environmental issue, news media is influenced by social, cultural and political factors. As a result, the coverage of an environmental issue does not necessarily directly correlate to the magnitude of the event. Magnitude itself is a contested topic.

\subsection{Comparing Perspectives in the Sociology of News Production}


Schudson (1989) explains the three major perspectives within sociology of news production and argues that even when viewed together, they do not provide an adequate explanation of the nuanced character of news production. The first perspective is the political economy approach. detailed above in the work of McChesney (2001), as well as Herman and Chomsky (2002). This approach directly correlates the production of news to the broader demands of maintaining neoliberal, corporate state organization. Schudson (1989) explains that because this perspective directly correlates corporate ownership with status quo results, it sees the conduct of news organizations as largely irrelevant. The second perspective is more grounded in sociological theory, and takes a sociological lens to the production of work and ideology, as detailed previously by Anderson (2002), Hannigan (2006), and Molotch and Lester (1975). This perspective attempts to understand constraints on journalists as well as how organizational routine contributes to the creation of the news. This perspective takes into account the viewpoint of the journalist, who generally considers him/herself to be autonomous, and does not dismiss this perceived autonomy as the product of a false consciousness. The third perspectiveless common in the literature and less relevant to this study — is a cultural approach, which looks instead at the influence of cultural tradition and symbolism rather than organizational routine.

Schudson (1989) critiques the political economy approach; he suggests that historically, a corporate-owned media serves the people better than an exclusively stateowned media. He cites instances when much of the mainstream media has covered antistate or anti-corporate causes, i.e., the Watergate scandal or critical coverage of the Vietnam War. He argues that this behaviour is inexplicable if shareholders and owners 
explicitly control the mainstream media. However, he suggests that sophisticated versions of the political economy account are crucial to understanding news production, the individualizing nature of news, and reliance on expert sources.

Like Schudson (1989), I suggest that focusing too narrowly on any of these camps invites oversimplification. While there may be some instances of direct corporate interference in media production, there are also institutional constraints that may result in a similar news product. Schudson explains that the political economy approach focuses on the big picture, while neglecting the finer details of news production. For this very reason, I have integrated both perspectives. The analysis chapters evaluate the extent to which the Deepwater Horizon spill fits within this range of perspectives, while also taking into account the finer details provided by a sociological lens, and keeping the large-scale issues of corporate ownership close at hand.

The above constraints on media lead to consequences in the news. Likewise, a corporate owned media will also presumably have effects on the news. The concentration of media ownership by corporations espousing neoliberal values has led to slashed budgets and intense competition for profit. Likewise, it has led to decreased disposable income for many potential readers. Shrinking budgets in many newsrooms may lead to reduced enthusiasm for investigative journalism, and reproduce the tendency to rely on available, trusted expert sources. The pressure to maintain profitability, as well as the constraints of adhering to the 24-hour news cycle, may lead many news media outlets to focus on short-term events rather than in-depth structural coverage. Molotch and Lester (1975: 255) use a sociology of news approach, but still insist that "news production cannot be understood apart from the political economy of the society in which 
it occurs." They explain that the increased access granted to oil companies and the federal government correlates with their societal power in general. Thus, they suggest that local economic conditions affect the likelihood of environmental events becoming public issues.

As discussed in the section on political economy, neoliberalism has a tendency to individualize social problems so as to minimize broader social responsibility. Likewise, the constraints of time and budgets, as well as adherence to organizational routine, may lead to stories that focus on individuals. Anderson (2002) posits that news stories, particularly ones that touch on environmental issues, may be individualized in order to fit into the 24-hour news cycle. Hannigan (2006) explains these same phenomena, but through the lens of event orientation. That is, news media focus on the events rather than the context in which they occur. This network of potential causes leads to stories as individualized as they are simplified. Stories with complex, systemic causes may thus be presented as personalized human-interest pieces (Jewkes 2004). Although personal narratives may be featured in the news coverage, these narratives are not connected to a broader system. The human-interest pieces may depict a heartbreaking personal story, but political and economic issues are presented as conflicts between various individuals (Jewkes 2004). Numerous theorists writing about the news have discussed "personification" (Ericson et al 1987; Hall et al 1978). These authors explain that the broader narrative of an event may be told through a select few individuals. This can have the effect of depoliticizing a story, since the focus is then placed on individuals, to the neglect of systemic issues. This trivializes the voices of ordinary residents and keeps their stories separate from the events of the day. 


\subsection{Kev Concepts: Frames, Ideology, Discourse}

Throughout my analysis, I employ many key concepts that require unpacking, and should be clearly linked to their theoretical context. Primarily, I use frames, ideology, discourse and narrative. Frame theory is the broader theoretical and methodological approach, but within this category, I employ the terms ideology, discourse and narrative as descriptors. While the details of my frame analysis strategy will be discussed in the methodology chapter, the purpose of this section is to discuss frame theory and debates within the methodological approach. As I discuss below, frame theory is divided into two broad camps, one which sees framing as an organizational tactic, and the other which views it as a system that reproduces powerful ideologies.

\section{Frame Analysis}

The first use of framing is organizational and unavoidable. In order to sift a coherent story from a mass of information, journalists must participate in framing. This explanation of framing is exemplified by Goffman (1974), who explains that frames give meaning to a series of events and are useful for making sense of large amounts of information. Frame analysis is not only used in the context of media analysis; framing may be discerned in other arenas of discourse. There are, however, media-specific applications of frame analysis. Gamson and Modigliani (1989: 143) define a media frame as a "central organizing idea or story line that provides meaning to an unfolding strip of events." Framing allows new information to be compartmentalized into familiar narratives. Without any frames, media would only be pieces of talk. Rather than conceptualizing the framing process as positive or negative, literature on frame theory generally considers the framing process to be an unavoidable organizational practice 
deserving of sociological analysis. Goffman (1974) highlights the usefulness of frames for organizing masses of information. He explains that frames are cognitive structures that are crucial to making sense of our worlds. It would be hazardous and fruitless to attempt to completely avoid framing because we actively interpret messages through our own sense of reality, and naturally organize information into frames or arguments. Entman (2007) explains that due to the overwhelming amount of events that take place every day, frames are inherently significant whether they are included or omitted. However, this usage of the word frame refers to the framing process that each reader, source, and journalist must undertake. In many ways, framing (as described by Goffman) is key to basic narrative thought; it is only applicable to this project as a type of background that facilitates an understanding of media's incapacity for strict objectivity. Framing involves making sense of events by individuals. Likewise, those individuals with a vested interest participate in the framing process and try to promote their pre-framed version of reality for public consumption. This second form of frame analysis is key to my thesis, and focuses on sources as framers. This focus can reveal differential access to media and power dynamics. Entman (2002) and Gitlin (2003) explain that frames influence public perception, particularly in the media. Mainstream media is a contested space where major actors fight for representation of their frame. Analysis of frames that occur in media can reveal the priorities and intentions of the framer and lend insight into public discourse. At times, frames also legitimate some points of view while marginalizing others (Gans 1979; Entman 2002; Scheufele 1999). Gans (1979) brings the discussion of framing more directly into the realm of power. Entman (2002) explains that control is crucial to understanding framing. and that the texts 
the media produce are actually imprints of power. Frames are often difficult to remove from the public once imprinted (Gamson and Modigliani 1989). Gitlin (2003) argues that media is able to turn the extraordinary into common sense. Although a media text can be interpreted in a plethora of ways, some readings are dominant; the qualitative researcher's role may be to extract these narratives, which contribute to broader framing packages.

Many frame theorists believe that frames are shaped by the perceptions of journalists, and that the reception of the frame is shaped by the perspective of the audience. As described above, members of the media must frame events, and thus framing involves the construction of reality. This thesis employs frame analysis in order to understand how some constructed versions of reality are prioritized. Thus, the analysis strategy requires an acknowledgement that frames are constructed and there is no knowable empirical frame. While the researcher or journalist cannot make assumptions about the effects of media, texts do have dominant readings, which researchers can attempt to distil through frame analysis. Although powerful institutions play a role in framing, many theorists suggest that members of the media are active agents (Scheufele 1999; GAmson and Modigliani 1989) and thus are unlikely to passively accept the viewpoints of large companies. One role of this project is to examine the extent to which this holds true in a contemporary environmental disaster.

The literature on frame analysis in media has been criticized for being too general and for possessing no consistent methodology (Scheufele 1999; Entman 2002). Although it is true that frame analysis cannot be easily reduced to a mechanical reproducible technique, the same is true for qualitative research in general. Just as there is no 
consistent way that frames become embedded in text, there is no single concrete way by which to distil them. Scheufele (1999) explains that within frame theory, there are distinct empirical camps. The existence of so many different approaches to frame analysis may render the label 'frame theory' too broad to be applied to a single methodology. Scheufele (1999) argues that the term 'framing' has been applied to a diverse range of methods. As I will elaborate on in the methodology chapter, contextdependent, qualitative case studies have tremendous value; Koenig (2004) argues that framing researchers should hermeneutically uncover frames. Thus, my research engages in this qualitative uncovering process. Efforts to combat the suggested vagueness in framing theory could strip the research of its context. Although frame theory allows for a variety of strategies and definitions, I focus on framers themselves. I also focus on how the frames of vested interests are promoted in news coverage.

\section{Frames, Discourse, Ideology and Narrative}

This section explains my use of the terms ideology, discourse and narrative in relation to frames. Frames are the cognitive packages that I look to detect. I begin my assessment of frames with sources; when sources promote or demote a point of view, this helps to define the limits of their frame. A source may have many frames, or may promote a specific larger frame almost unilaterally. Likewise, journalists participate in framing through the research and writing process. It is of no benefit to this project to determine whether a frame is a large or small idea; a frame is any viewpoint that a particular actor or organization advances, and it depends strongly on what view they suggest. 
In this thesis, I use discourse to mean pieces of talk. This term has often been used in fields such as Critical Discourse Analysis to mean "utterances" as well as the overarching ideas and narratives that connect them (Fairclough 1995 a). I find it more useful when employing frame theory to define discourse as uttrances and pieces of talk rather than as overarching narratives. Connected discourses can be constructed into narratives; key narratives that are promoted by different sources are frames. In general, I only discuss discourses only when they can be connected to a broader cognitive framing package, by either supporting that frame, or dismissing it.

Frames generally promote or demote an ideology. I use ideology to politically situate the frames uncovered in the research process. Ideology is made of discourses that have generally been assembled into narratives. Ideologies support or dismiss an idea or way to live. Eagleton (1991) explains that ideology is not inherently false and it need not involve a false consciousness. Many ideologies are perfectly true, while others are not. A classic definition of ideology is a system of ideas that promotes, protects, and legitimates a dominant group and the status quo (Eagleton 1991). A great deal of the ideology I detect in my research legitimates systems of power, but contesting ideology is also included. For the purposes of this investigation, I take the view that ideology and discourse are in no way opposing forces-nor are they interchangeable. Rather, discourses may construct an ideology, and ideology is always made up of discourse. They are compatible yet distinct branches of theoretical inquiry (Purvis and Hunt 1993). These authors explain discourse as process and ideology as effect (494). Ideology always has definite effects; if an ideology has no impact on anyone or anything, it is not an ideology at all, but merely an idea. Ideology always has a discursive form, but discourses need not 
be ideological. My thesis is not inherently concerned with all ideologies, but rather by the promotion or demotion of ideas from key sources (which constitute their frame).

Oliver and Johnston (2000) explain that some framing theorists have problematically reduced ideology to a component of frames. Ideology has been defined differently by many scholars, but is a well-established and useful term in the social sciences. Rather than formulating ideology as a component part of a frame, I situate frames within their political context by considering the broader ideological forces that shape their creation. Frame analysis is an extremely useful media analysis strategy, and frames are my focus. However, if my thesis ignores the broader ideologies that shape and contribute to every component piece, it would also neglect the sociological richness of the investigation.

In sum, the frames I detect are always made up of discourses and often convey an ideology; some frames are extremely broad ideologies themselves, and others indicate a still broader ideology. A discourse can be meaningless on its own, while an ideology is constructed of a chain of narratives, and promotes or demotes a system of ideas. When those narratives come from a source in my sample, I describe this as a frame. Frames are likewise constructed, shaped and displayed through the journalist's writing process.

\section{$\underline{2.5 \text { Conclusion }}$}

This chapter summarized much of the debate over the reasons why news media coverage exhibits certain perceived shortcomings. Media products tend to attribute events to individuals rather than to more complicated social problems. Likewise, these products tend to focus on events in the immediate present, rather than connecting them to economic, political and social history. Consequences often include trivialized personal 
narratives rather than a broader consideration of social and environmental issues. This chapter prominently featured the contrast between those who see the media as an arm of elite ideology (by merit of corporate ownership), and those who see journalism as shaped by a vast array of constraints and influences (which may at times provide openings for challengers to the status quo). In the analysis chapters to follow, I aim to integrate a broad analysis of political economy with the understanding that the corporate role in media is not all encompassing; the lived constraints on journalists affect their choices and how they construct the news.

The theoretical frame presented in this chapter explains, through various viewpoints, that the products of the news media often individualize broader social problems and rely on expert and official sources. Using frame analysis, I will engage this literature to answer my research questions and explore these tendencies with reference to a contemporary environmental disaster. This project advances the literature on sociology of the media and political economy by exploring news media responses to an accident that was arguably the result of a degraded regulatory climate and neoliberal economic macrostructures. 


\section{CHAPTER III}

\section{Designing a Methodological Approach to Studying News}

I have established that news coverage of a major environmental event is a clear opening for potential research. My next challenge involves how to study it. That is, how much data should I examine? From what time periods and sources should I derive this data? Which methodology should I employ? This study is based on a content and frame analysis of online news media coverage of the $2010 \mathrm{BP}$ oil spill. The data consists of online content from the New York Times in the nine months following the spill. Specifically, I analyze stories taken from seven key date ranges that sample the reporting of the progression of the spill.

This chapter details my methodological approach to gathering and analyzing the data in 70 news articles. The first phases of analysis involved a broad overview; I counted, quantified and assessed the voices in the media sample in order to map access. For the second phase, I immersed myself in the data in order to detect dominant frames and their indicators, which eventually revealed the final incarnation of my argument. Young and Dugas (2011) focus on voice and narrative in their use of frame analysis. This format worked well with my project, which also contains two layers of analysis. The first layer is voice, which involves cataloguing who has access to the media and analyzing the general content of these voices. The second layer involves thematic organization and analysis of the frames I detect in the sample. Young and Dugas use a similar technique, but rather than frames (my focus), they describe sifting narratives in their sample. During this process, my argument evolved. Initially, I suggested the news material narrowed 
expansive social and political problems. Later, I came to suggest that the sample reproduced neoliberal ideology.

I have constructed a defendable strategy of purposive sampling, which I detail in the chapter below. I begin by justifying my decision to study media and online news. I then explain the specific challenges presented through online news websites. I use these challenges as a venue for summarizing my data collection strategy. I then explain the benefits of a case study design and my rationale for content and frame analysis. Finally, I detail the complex process of data analysis as it was conducted in each of the data chapters. I focus on voice and access; I quantify the access of different sources, explain the significance of this access, and explore the content of their access, which is necessary to understanding the relative value placed on different sources. I summarize the second layer of data analysis as content and frame analysis. I detect the broad framing packages present in the data by linking individual discourses to narratives, while focusing attention on those narratives promoted by key sources. I then explore the connections between the broader framing packages that emerge in Chapter Five, and the construction of sources, as explored in Chapter Four.

This research is exploratory and begins with broad questions about media, news sources, and the political economic climate. My research questions include: How are environmental accidents framed in news media? Does that framing process echo trends in the broader political economic climate, and if so, how? From there, I also explore the portrayal of key sources, who are often the focal point of news stories. This project aims to take a holistic view of the material in a key sample of news stories in order to better understand the spectrum of information presented in the news media following an 
environmental accident. My case study design is uniquely prepared to answer these broad questions.

\subsection{News Media}

Investigations into media have occurred nearly as long as the media has existed. Bell (1995) explains that media is of particular interest to researchers in part because they are a rich source of easily accessible data. He explains that products of the mass media reveal social meanings, and that media simultaneously reflects, interprets and influences our politics, culture, institutions and social lives. News media provides the first and most readily available reaction to the $\mathrm{BP}$ oil spill, and it is arguably the source that had the greatest effect on shaping public opinion. Molotch and Lester (1975) explain that the overarching purpose of media analysis is to study the nature of event making as a social and political activity.

Media is a crucial site of investigation into environmental issues; it is one of the most immediately researchable areas by those in the social sciences. Many studies look specifically into the relationship between the media and environmental issues (Hansen 1993; Anderson 2002 and 2007). Within Sociology, there are a many viewpoints from which to research the Gulf spill, including interviews with corporations, government, or residents of the Gulf. It can be argued that there is no other potential data source that factors as much into public understanding of the spill as news media.

\section{Online News}

As I will discuss shortly, my choice of online newspaper websites has presented many research challenges. Such organizational and logistical barriers may discourage researchers from moving their research online. There are many signs to suggest that 
newspaper distribution is decreasing (Antilla 2005) and will eventually become obsolete; readers will not necessarily turn to online subscriptions. Many readers consume the day's news as it breaks, using RSS feeds, Google News, personalized Google alerts, and the activity of social networking contacts. Often, readers in turn use social networking to share the stories they find most compelling. A paid subscription to a single news website is impractical for those who want to share and read from the spectrum of news available online. This new fluidity in news readership may be difficult to track, but is nonetheless significant. Although it is doubtless valuable that many researchers continue to track coverage in physical papers, it is likewise important that some begin to explore the methodology required of online research.

The print and online versions of most papers are often very similar, although some analysts argue that the materials are received differently due to the change in medium (Schonbach, Waal and Lauf 2005). Rather than looking at alternate sources of web news, I focused on the online version of a major paper. Print newspapers are a widespread, well-recognized and trusted news source for many people and are likewise valuable for research. Print is a material form of news that is often trusted for similar case study designs (Molotch and Lester 1975, Anderson 2007). As I will discuss shortly, there is special significance in selecting the New York Times when tracking the media coverage of an American event.

Regardless of whether it arrives in online or print format, the mainstream news media is still the primary public source for information on environmental issues (Young and Dougas 2011, Antilla 2005, Hannigan). The public is awash in media information, and increasingly depends on experts who are able to disseminate complicated scientific 
and environmental events (Anderson 2007: 98). Alternate sources such as weblogs have captured public attention, but their distribution rates and patterns do not compare to conventional mass media. Alternate sources rely heavily on mass media for their content, and are not perceived to be authoritative or legitimate sources for news (Young and Dugas 2011). Online access is increasing constantly and spreading into the daily lives of more readers as many news organizations shift their focus to digital coverage.

\subsection{Case Study and Sample}

My research process began with questions about how environmental incidents are portrayed in the news. This format is well suited to answer my research questions: how are environmental accidents framed in news media, does that framing process echo trends in the broader political economic climate, if so, how? These questions are appropriate for a qualitative case study, because such case studies are effective for exploring 'how' questions, to which there is seldom a single unified response (Flyvbjerg 2001; Gray 2004). Although case studies have been the subject of criticism, Flyvbjerg outlines and dismisses major misunderstandings about case study research (2001: 66-87). He explains that for many research questions, case studies are desirable, effective and more valuable than alternatives. While cases produce context-dependent knowledge, Flyvbjerg (2001) explains that it is possible to generalize from a single case, and efforts to strip research of context only damage the overall presentation and dilute rich circumstantial information. He argues that effective and thoughtful case studies produce context-dependent knowledge and that case studies mirror the complexities of life.

There are three layers to my case selection and sample; all are informationoriented (Flyvbjerg 2001). The first layer is the broader case itself: an environmental 
disaster and the news coverage of this event. Flyvbjerg (2001) separates cases into types, and I will use the oil spill as a hybrid of his extreme and paradigmatic cases. The extreme case, like Foucault's Panopticon, can be "well suited for getting a point across in an especially dramatic way" (Flyvbjerg 2001, 78). There is specific theoretical importance in my choice of the worst and most recent oil spill in American history. The US itself represents an extreme case of a shifting global economic macrostructure, and the BP oil spill represents a timely, extreme, and highly publicized case of environmental degradation. Flyvbjerg (2002) explains that the typical case is seldom the richest in information, so I look instead to a case that involves a cluster of extreme circumstances.

According to Flyvbjerg, the researcher can even generate general propositions and theories from case studies, which is the aim of the paradigmatic case as well as one aim of this project. The BP spill serves as an exemplar: a catastrophic environmental accident that was at least partially due to a failed regulatory structure and move to neoliberal economics, as discussed in Chapter Two. Likewise, the United States is arguably the birthplace of neoliberal economics and an exemplar for this ideology. The United States also has the single largest national economy in the world. Thus, although the BP spill is an extreme case, I use it to make statements about the types of knowledge and priorities that are informing contemporary media coverage.

The second layer of my sampling strategy falls within this broader case. I have decided to look at the online coverage of the New York Times, which was selected because of its important historical significance. As will be discussed in more detail shortly, it is renowned as an agenda-setting publication and is the most politically liberal, national, mainstream paper in the U.S. I have selected another extreme case by using the 
most liberal and influential mainstream newspaper in the United States. On this smaller scale relative to the broader case, the sampling strategy resembles a critical case, which Flyvbjerg (2001) describes as: "having strategic importance in relation to the general problem" (78). He explains that the researcher can then make logical deductions: if the New York Times does not feature an in-depth critique of the spill, I will be unlikely to find one in other mainstream US newspapers.

A detailed, information-oriented sampling process informs the third and final layer of my sample (Flyvbjerg 2001, 79). This process involved selecting a seven-month time frame following the spill, as well as selecting particular news days within this window. Rather than being extreme or paradigmatic, this layer of the sampling is organizational and designed to maximize the variety of information from a limited sample. Without the resources to sample all news that followed the spill, I must place parameters on the range of coverage. This is a necessary step in a project of this scope.

\section{Selecting the Sample}

My sample consists of American news because the spill occurred in the United States and I do not comparing how the news was presented between countries or internationally. Anderson (2002) confirms that media production is influenced by national cultures, so the coverage of the BP spill may vary among countries. To extend my sample beyond the United States would inevitably place analytical focus on the difference between different national versions of coverage.

The New York Times is often referred to as an agenda -setting paper in American news. It is nicknamed the Gray Lady for its role in American culture, and is referred to as the "newspaper of record" (Marshall 2007). The New York Times is owned by the New 
York Times Company, which includes a number of other prominent papers and media sources in the United States. It ranks third in paper circulation, but is the most visited online newspaper in the United States, and has the most online subscriptions (Newspaper Association of America 2011). The New York Times is an ideal site for investigating an American event, particularly when studying online media.

I had originally intended to research The New York Times, The Wall Street Journal and USA Today, but quickly discovered that these three papers often overlapped. USA Today coverage frequently includes shorter versions of stories from the New York Times. My initial decision to study multiple papers was motivated by a suspicion that there would be distinct differences in coverage that must be accounted for, leading to a comparative analysis. Given the strong similarity between coverage from different sources, the comparative element was not a rich one for investigation. It was more significant to my research questions if I examined a broader sample of news days from single media outlet. This strategy expanded the sample coverage to a more diverse range of events and discussion on the oil spill, rather than featuring many similar stories on a narrow range of events. Many media scholars agree that The New York Times is arguably the most politically liberal national newspaper in the United States (Young and Dougas 2011). I intend to detect the extent to which mainstream coverage engages with systemic issues; the most politically liberal paper is the most likely source for in-depth systemic critique. Likewise, if this critique is omitted from The New York Times it is even less likely to appear in other mainstream national news sources.

Early in the research process, I decided to focus my media sample on key dates in the progression of the spill rather than attempting to create a random sample. The oil spill 
and clean-up can be seen as one overarching event of a long duration, but this arc is punctuated by smaller events that often define its immediate representation in news. This characteristic is discussed in the media section on event orientation in Chapter Two (Anderson, Molotch and Lester, Young and Dougas 2011). In the seven months following the initial explosion, some days featured no mention of the spill. I used key short-term events to orient the general date ranges, but I subsequently collected all BP related news in a five-day window around such short-term events. I discuss the collection process in greater detail below. The events I focused on are oriented around the following dates:

1. April $21,2010-$ initial explosion in the Gulf

2. May 8,2010-first attempt at plugging the leak fails

3. May 29,2010 - Top Kill procedure fails

4. July 27,2010 - BP CEO Tony Hayward is replaced by Robert Dudley

5. August 4,2010 - static kill procedure is a success and oil stops flowing

6. September 8, 2010-BP releases their internal report on the spill

7. January 5, 2011-the Presidential Commission finalizes their report on the spill Each of the stories in my sample is associated with a number, which refers to one of these seven groups. I initially decided to focus on just five key events in the progression of the spill; I believed that this number would provide me with a reasonable sample. As I examined the news coverage further, I selected two more events because I wanted to include adequate coverage from the months the wellhead spent leaking. I also wanted to include news coverage that corresponded with the release of spill reports from $\mathrm{BP}$ and the US government. It was important to my investigation to include stories that corresponded 
to these reports because I am specifically interested in the political economy of news production and how the frames of the powerful are reproduced in mass media.

The placement of each date range depends on the specific event. For example, the top kill procedure failed on May 29, 2010. That day was a climax in coverage, but the procedure itself began many days earlier. The event was thus discussed in the news as a plan, a process, and then as a failure. I subsequently decided to shift my five-day window to May 28 - June 2. In contrast, my sample for the initial explosion begins on the date of the accident, as there was clearly no foreshadowing or 'rising action' in the news. I exclude all opinions and blogs, but include relevant stories that are cross-listed in sections such as finance or travel.

My sample includes all BP stories in the five-day range, including hard news and feature stories. I had originally intended to focus on hard news, but I realized that I was neglecting an entire realm of feature news coverage. Bell (1995) explains that hard news and features are the two principal categories in news media. Each generally adheres to its own format, although there can be grey area between the two. Bell suggests that hard news always begins with a focus on immediate events. Feature stories often lack this focus and tend to be longer. The feature format allows the author to follow a narrative, which is typical of 'soft' news. Bell further explains that feature authors often take more liberties with their writing style and cover more background.

Pan and Kosicki (1993) describe an inverted pyramid format that characterises hard news. This style begins with the most recent and relevant information, providing detail and background along the way. Feature stories often begin with a narrative lead and progress towards the climax of the story, whereas hard news aims to immediately 
inform the reader. In hard news, information becomes less thematically and temporally significant as the story progresses. I elected to include feature stories because they may examine a more diverse range of topics related to the oil spill and provide openings to alternate frames and more analytical or critical coverage. Hard news stories are equally significant. Thus, it would do a disservice to my research if I restricted the analysis to one format or the other.

To compile my stories, I searched the online database of The New York Times using the search term 'BP Oil,' which returns a large result and includes stories that are only tenuously related to the spill. I then assessed the diverse array of individual articles to determine whether the topic was actually the spill. In one story, Berkeley students considered cutting their ties with the oil giant. In another, BP gas stations around the United States noticed falling sales. Due to the broad nature of the stories, it was impossible to generate the sample from a few specific search terms alone. Instead, it made more sense to take this broad sample of all BP stories from all sections of the paper, excluding opinion pieces. Within that broad sample, I read each story to determine whether the topic was the spill itself, or just a related event. When dealing with online versions of newspapers reporting one of the biggest national events at the time, the researcher must use his/her judgment in order to collect a manageable sample for a research project of this scope.

Within the broad case of the oil spill, which I will discuss more thoroughly in the following section on cases, I decided to sample the New York Times online coverage of this case. Within this coverage, I have sampled seven key periods of high coverage that correspond with major events in the progression of the spill. As a result, I needed to make 
decisions about how to sample within those seven events. The events are used as windows of time that feature high-volume coverage; I have not restricted my media sample to hard news coverage. Instead, I broadened it to any other stories that occur within a designated window of time. This qualitative project benefits from the use of my research instincts and careful reading of the sample.

\subsection{Voice and Access}

This section summarizes my methodological approach to the first half of the data analysis. I focus on voice and access. Voice refers to the content of quotations and paraphrases, and access refers to the volume of quotations. I quantify the access of different sources, explain the significance of this access, and explore content of their quotations. I refer to featured actors who are quoted in a story as sources. These sources often inform the framing of the article, as will be discussed in the second section of my data analysis.

Many media scholars agree that quotation patterns reveal who has access to the media and whom journalists trust as expert sources (Anderson 2002; Hannigan 2006; Molotch and Lester 1975). Although the complexities of voices and their messages will be extensively explored in the frame analysis, quotation patterns reveal how different sources are valued and prioritized in news coverage. The process of organizing quotations and paraphrases into categories and sub-categories has been a richer and more complicated process than I anticipated. This section details how I organized the participants quoted in the 70 articles. I categorized and tracked all of the sources who occur in the articles and totalled their representation. I also kept track of who is quoted 
first in each story. This data was then compiled into tables. I explain the creation and organization of the tables in the section to follow.

Deciphering Sources and Attributing Quotations

The four tables contain categories and subcategories which I created as an organic response to the quotation patterns through analysis. I did not begin with a predetermined list or set of main categories before beginning data analysis, although I did have a general sense of the sources present in the articles from earlier readings. I have created four tables to display and quantify the quotation patterns in the articles; see Appendix A (Table One), Appendix B (Table Two) and Appendix C (Tables Three and Four).

Within these tables, I have organized the quoted participants broadly into the following categories:

- Locals and Fishermen

- $\mathrm{BP}$

- Business and Corporate

- Federal Government

- BP and Government (quoted as one)

- Anti-BP Sources

- Experts

- Environmentalists

- Other and Unattributable.

Across the top of the first table (Appendix A) are numbers that represent each of the 70 articles. For example, 2.24 denotes range two, Article 24. Table One has a second section at the bottom that shows who was quoted first in each article. Table Two (Appendix B) 
compresses data from Table One by adding the total number of times each major category is quoted in the sample. Table Three (Appendix C) repeats the sums for total quoted in each major category. It also shows the number of total articles within which each major category has at least one quotation and the percentage that this number represents. Finally, Table Four (Appendix C) shows the totals from the second section of Table One. That is, the total number of times sources from each major category were the first quoted and the percentage of total articles it represents.

The most challenging component of this process was deciding how to categorize the quoted participants. I use the term quotation broadly to include paraphrases as well as direct quotations. I found it difficult to decide what counts as a quotation. At times it was unclear whether a journalist was actually paraphrasing members of a group or only inferring the general position of that group; this situation required careful consideration of context. For example, Article 10 reads: "And while most environmentalists support the application of dispersants as a necessary evil to limit the damage, some have assailed an industry policy that guards their chemical makeup." In this case the journalist is inferring a general position rather than paraphrasing a particular environmentalist group.

Many quoted and paraphrased actors in the articles were cross-listed between categories. For example, Article 48 cites founder of the Center for Biological Diversity. Although this speaker may have been a scientist or engineer, I classified the quotation as an environmentalist, since the primary identifier was an environmental organization and the founder spoke out against a lack of government regulation. Many of the quoted participants and organizations belonged to more than one subcategory, and I sorted them according to the context provided in the article and the substance of their quotation. 
I often conducted background research on quoted organizations because the quotations did not always reveal the type of organization. Although the name of an institution may make it sound like an environmental group, such groups are often funded by the oil industry. For example, Articles 2 and 34 quote the Energy Policy Research Foundation, which is an industry-funded research group. It was often unclear whether spokespeople for university- and college-affiliated research institutes were scientists, biologists, etc., or speaking on behalf of the oil industry. Article 62 quotes the program director of the Petroleum Extension Service at the University of Texas, who speaks positively of the Halliburton cementing job. The Petroleum Extension Service is located within a university but receives no government funding, researches on behalf of the industry, and has an advisory board primary composed of industry representatives (PETEX 2011). The speakers for these groups are often program directors, and their academic credentials are left unspecified. I thought it best to separate these oil-funded researchers from other biologists and scientists and created the energy/petroleum subcategory.

Although I did my best to ensure quoted participants all fit into at least one general category, an 'Other' column was unavoidable. For example, Article 63 has the highest volume of 'Other' quotations in a single article. It features ten quotations from various journalists. The article describes how news coverage was beginning to assess the spill impacts rather than describing the spill in progress. National news anchors could perhaps otherwise be classified as experts, but it would be counterintuitive to list members of the media as experts when all others in this category are scientists and academics. I separately counted unattributable officials and analysts as a subcategory 
within the Other category, as it is significant to isolate how often journalists fail to attribute a quotation or paraphrase to a specific organization or person. Additional category-specific and sub-category-specific organization is discussed below in the analysis of the data.

\section{Table Composition}

The design and composition of the tables has a strong effect on how the quotation pattern data reads. The major categories were generally self-evident (i.e., locals, BP, federal government, experts and environmentalists). Initially, BP was categorized as a subheading under a broad corporate/business category but I decided to subdivide the corporate category in order to isolate BP's media access. A number of organizations and groups are broadly included under both "locals" and "government." Although a corporate/business category gave a good sense of how often non-local business voices were heard, I thought it was important to refer specifically to how often BP was quoted and compare BP's access as a major subcategory against locals and government. BP is a specific corporation and one of the largest stakeholders in the disaster, arguably second only to the residents of the Gulf coast. Within BP there are a number of subcategories, including their employees and engineers.

After I have separating the BP voice from business and corporate, they appeared to have comparatively lower access. No other single stakeholder, company or organization is represented as its own major category. BP is separate from other corporations like Transocean and Halliburton, while any business, resident, politician, fisherman or NGO from the Gulf coast are categorized within the local category. As a result, it is somewhat unrepresentative to directly compare the local voice to BP. This 
difficulty illustrates the importance of the second stage of data analysis; although it is helpful and important to quantify the quotation patterns, it is only a preliminary step in this project.

I chose to subdivide Local into finer detail than BP or the federal government because there is a diverse variety of institutions and individuals within the broad Local category. Local business owners and fishermen may have completely divergent views and priorities, whereas all BP spokespeople reflect a generally unanimous voice. For example, the incoming BP CEO, outgoing BP CEO and BP's President of Gulf Operations all echo nearly identical arguments and promote the same general frames. Unsurprisingly, the residents and employees within five states have more diverse interests to convey than the spokespersons for a single corporation.

I chose to count the quotations according to the number of paragraphs in which they appear, never to exceed one quotation per paragraph for a single person or institution. For example, if President Obama is quoted three times in a single paragraph it counts as one quotation, but if he is quoted in three separate paragraphs within the same article, it counts as three. By counting the quotations this way, the numbers indicate to some extent the content of the article and how many different sources are featured in each article. Paragraphs in the articles are generally very short, but if I did not continue to count the same quoted participant when he or she was mentioned again in subsequent paragraphs, I would lose the makeup of the article; the same weight would be given to a speaker who was quoted once as a speaker who was continually quoted for five paragraphs. This strategy best provided a sense of the content of the articles. 
Certain articles cite BP and Transocean employees who wish to remain off-record in order to speak out against the practices of their employers. BP clean-up workers were prohibited from speaking to the media, and some employees are cited in the context of trials involving the outcome of the spill. As well, some journalists cited damning pre-spill BP documents and records. The presence of these voices necessitated a separate category for dissenting voices within the company: anti-BP and Transocean sources. Although I could have simply counted these quotations as BP or Transocean employees, part of the goal of this project is to decipher the extent to which corporate voices like BP are represented in news coverage and control its message; these dissenting voices presumably do the opposite.

It is not useful or significant to total the number of articles within which every subcategory has a quotation or paraphrase. Different subcategories within the local category will often be quoted in a single article, i.e., residents, fishermen and local politicians. BP is not subdivided into so many categories, but likewise, many representatives may all be quoted in a single article, i.e., the CEO, a spokesperson, the president of Gulf operations and incoming CEO. Thus, if I were to total all of the subcategories, this would count as access to one article for BP, and for locals it would count as access on behalf of all of those separate groups. Thus, Table One (Appendix A) shows the composition of each article by subcategory, but I only sum the access for the broad categories (Table Two in Appendix B). I had originally counted access on behalf of every subcategory group, but subsequently changed my method and counted only within the larger category. Table Three (Appendix C) displays the nine major categories, total 
number of times quoted, and the number of articles in which each major subcategory appears, as well as the percentage that this total represents.

\section{Voices}

This portion of data analysis, which occurs in Chapter Four, summarizes and analyzes the content of the featured voices rather than only focusing on how often they occur. Through careful reading, I compiled quotation patterns and summarized examples for in-depth discussion. I focused this method on key examples that illustrate the frames of a group. More importantly, I focused on examples that illustrate a framing trend or indicate a broader ideology. The frame may be expressed through a focus on industry and jobs, or through the belief that the government is failing to protect its citizenry. This frame could be linked to broader ideological packages of neoliberalism and the free market, or alternately the promotion of a social welfare state. I categorized every quoted or paraphrased participant and assembled this in Table One (Appendix A) and could therefore sift easily through the articles and read only the sections that had been coded as belonging a certain group. Thus, I was able to synthesize the voice of each group as it was portrayed and detect the common frames as well as outliers. The analysis focuses primarily on locals, BP, environmentalists and government.

The analysis of voices functions as a crucial link between the quantitative data represented in the tables, and the broader frame analysis conducted in Chapter Five. The voices section provides key insight into the construction of sources by the media. It was important to consider the general narratives presented by key sources rather than only organizing this information within broader framing packages.

\subsection{Content and Frame Analysis}


In this section I explain the details of how and why I conducted frame analysis in Chapter Five. I detected the broad framing packages present in the data by linking individual discourses to broader narratives. When narratives are advanced by a source, it becomes their frame. Content analysis is the most commonly used method for detecting frames (Gans 1979; Semetko and Valkenburg). Most researchers who use frame analysis identify this methodology as a component of content analysis. (McCarthy 1994; Gans 1979; Semetko and Valkenburg 2000). I specifically use contextual content analysis as a broad methodological approach within which I employ frame analysis. Contextual content analysis focuses on an interpretive approach rather than a quantitative overview, and stays focused on the qualitative context in which information is constructed. Bonfadelli (2007), McCarthy (1994), and Semtko and Valkenburg (2000) have all used this approach. Essentially, it is a way to divorce content analysis from its associations with quantitative approaches and ground it unabashedly in an interpretive process. I have adopted Koening's (2006) stance that framing researchers should hermeneutically uncover frames.

Although the background literature on frame analysis was explored more thoroughly in Chapter Two, I will briefly discuss why this is an effective and appropriate methodological approach. Callaghan and Schnell (2001) explain that "political elites can effectively use frames to promote their own political ends," and that "we know less about the ability of political elites to put forth and promote their frames than we do about the influence frames exert on public opinion" (186). Frame analysis of news media has the capacity to reveal the representation of elite frames in media and simultaneously begin to decipher the role this media plays in public perception. Thus, my investigation 
contributes to the literature by exploring how different source groups (including political elites) put forth their frames, how journalists chose. construct and present this information, and the subsequent effect on the overall coverage.

\section{Using Frame Analysis}

Careful reading of documents from the government, BP and environmentalists provided me with background on many of the competing frames and broader ideologies that I subsequently detected in the news stories. This work reveals the dominant and omitted frames as present in news coverage and allows me to determine whose frames occur most frequently. I compile frames through immersion in the data and by identifying and cataloguing the contested issues, with a particular focus on those issues that continually reoccur through the layers of coding.

Rather than conventional line-by-line coding of the text data, I carefully read for the presence of reoccurring narratives. Entman (2002) explains that frame analysis is an intuitive process that rests largely on the judgment of the researcher. I do not argue that framing an event is necessarily harms the output of the media, but rather look to understand the content, motives, and political orientation of the resultant frames. Frames are often explained as broad theoretical packages, without which the content of media would be meaningless (Goffman 1974), but I am only interested when a particular source or the journalist promotes this theoretical package. Consider a broad frame, for example: increasing regulation will harm industry. This frame can be detected within many articles at different levels from different sources. BP, the federal government and industry all generally advance this frame. The frame that regulation will harm industry stems from neoliberal ideology that prioritizes the growth of the market over all other social needs. 
Although I remained open, I began the research with some background knowledge of the different narratives that may be advanced by key sources. Thus, I have connected individual piece of discourse to broader cognitive packages (frames) by using my preexisting knowledge of social narratives.

When coding the documents, I listed the general contested issues that I believed I would be looking to detect, but likewise remained open to the presence of new and unexpected frames that could emerge from the data. My codes were not strict, static, or concrete. I also took note of specifics that seemed important and quotations or paraphrases from groups that warranted further discussion. The coding process circulated around issues of blame, causality and solutions. I only coded relevant information, but frequently went back to the data, remaining open to the notion that new contested topics would arise. After carrying out the above data analysis strategies, I explore the connections between the broader framing packages that emerge in Chapter Five, and the construction of sources as explored in Chapter Four.

Frame analysis is an appropriate research method for understanding meaning as constructed by various sources. I coded the articles for word choices and indicators that relate to several broader frames, while attempting to summarize the general frames present in each article. In the first range of my sample, the explosion had only just occurred, and although interesting material is communicated, there are not yet broader frames being promoted by a particular group; at this point in the spill progress, there was little struggle over how to frame the spill beyond BP and Coast Guard officials suggesting that the well was unlikely to be leaking. Eventually it became clear that a major public relations struggle in this kind of a disaster is related to attribution of 
responsibility, and thus prominent frames are those of blame. I also attempted to uncover the extent to which the stories discussed policy implications such as the benefits of a drilling moratorium, the benefits and drawbacks to deepwater drilling, and the merits in reducing fossil fuel dependence. Particularly in the first sample, it is unlikely for the discussion to go to this level as it was predominantly focused on the events surrounding the explosion and the missing rig workers.

One objective of this project is to examine the extent to which these more complicated social questions arise, and when they do, which perspective is being promoted. In the case of some environmental issues, their public debate includes two clear perspectives (i.e., whether or not to drill in deep water). This division becomes more nuanced when framed in the context of an environmental accident. In the case of the spill, there is no explicitly positive 'pro spill' side, since it is unlikely any source would admit to being in favour of oil spills. Thus, it becomes the researcher's job to detect efforts by certain voices to minimize the long-term repercussions of the spill and implicitly forgive $\mathrm{BP}$ or the government for their role in the disaster and clean up.

The analysis occurred in two levels; the first, which was based on coding the articles for evidence of sources placing blame, projecting a particular solution, focusing on individuals at fault, etc. After completing this process, with the emergent codes in mind, I immersed myself in the data once again and identified thematic links between the prominent codes. At this higher conceptual level, I uncovered an underlying context that united many of the prominent frames: the prevalence of neoliberal ideology. This was observed through a tendency to prioritize the economic and business solutions to the spill, minimize the magnitude and potential impacts of the spill, and individualize systemic 
economic and social problems. These frames will be explored in greater detail in Chapters Four and Five.

\subsection{Conclusion}

Content and frame analysis are appropriate research methods for a qualitative case study on media reactions to an environmental accident. The oil spill itself is an extreme example of environmental degradation in a country that best embodies neoliberal economics. The New York Times is an appropriate venue for examining the national response in the United States, and my selection of seven key events is designed to maximize the relevant information in the sample, while taking all relevant news on those days. I take a closer look at the voice and access of key sources as well as the broader frames, which are present in the stories. This design is appropriate for answering my exploratory research questions that aim to make sense of how media are constructed, particularly in the wake of an industrial disaster.

I began this investigation intending to take a bird's eye view of the data in the articles and I did not solidify an argument until after the data analysis was complete. I began my research with the observation that the coverage seemed to individualize the

spill, and suspected this may be symptomatic of neoliberalism. However, as I assembled the frames and themes it became clear that while the tendency to individualize was prominent, there were other equally important emergent themes, thus the argument flowed organically and clearly from the data. For these reasons, this project is uniquely prepared to answer my broad research questions. My argument emerges clearly in Chapters Four and Five on data analysis. 


\section{CHAPTER IV}

\section{Voice and Access}

This chapter details the initial stage in the data analysis, which is an overview and analysis of quotation patterns. The data is revealing in terms of who has access to expressing their viewpoints in the sample and at what volume. I also look at voices, which are the narratives or frames of those with access. It is important to outline how access is distributed in the news articles because it allows me to explore the extent to which this news sample reproduces the oft-cited reliance in journalism on authoritative and expert sources, primarily from trusted organizations (Molotch and Lester 1975; Anderson 2002; Herman and Chomsky 2002). A key interest of this study is to evaluate whether alternate voices can effectively access openings in the media landscape during an environmental accident, or if authoritative sources like governments and scientists are prioritized. Consequently, this chapter features a strong focus on the prominence of expert and authoritative sources versus alternative sources. The chapter also unpacks how the methodological organization of the data has influenced the way the data reads.

The chapter begins with an examination of the categorization process, and how this shapes the data to follow. I then analyze the data presented in the tables and give examples to illustrate the trends in source voices, and discuss the significance of their content. I explain the organization of the tables and categorization. I briefly describe the process of compiling quotation patterns, and discuss the significance of an analysis of access through quotes and paraphrases. I then discuss patterns and observations that have emerged from this first layer of analysis. This immersion in the data set the stage for the frames and themes that emerged from the overall project. 
The final section of this chapter discusses the voices of locals, the federal government, environmentalists, experts and $\mathrm{BP}$ with attention to the detail of their statements. The chapter features a strong focus on locals, who represent a diverse group with a large overall voice, and whose portrayal contributes to a greater understanding of the state of news media. The chapter sets the stage for Chapter Five by taking a close look at sourcing patterns and the narratives advanced by these sources, which I refer to as frames. The relationship between sourcing and frames is reinforced as specifically relevant to oil spills: "where there is considerable scientific uncertainty, issues of access and hierarchies of source credibility are crucial to understanding different framings" (Anderson and Marhadour 2007: 112). It would be shortsighted to extract the frames in Chapter Five without examining how the different major sources were constructed in the news coverage since it is these sources who generally promote or demote a frame.

\subsection{Overview of the Categories}

In this section I explain the challenges of designing each category and subcategory while organizing the tables and dividing the sources present in the sample into categories. This discussion goes beyond a conversation about methodology and delves into the ways that categorization becomes a component of analysis. I briefly discuss general observations about the quantity of quotes from the sources, but this will be discussed more explicitly in the following sections on the tables, and the themes within the voices.

\section{Locals}

The categorization of locals presented specific challenges. As I conducted the research I was faced with deciding to what extent I should subdivide Gulf residents, 
workers, business-owners and businesses into separate categories. Locals are not a homogeneous category and are thus difficult to analyze as a single unit. Local politicians. attorneys and businesses are authoritative voices that are often privileged in media and were thus first to be subdivided from general locals. As I continued with the research I decided to separate rig workers. They are technically BP and Transocean employees, but have a unique voice in that they were present on the rig when it exploded and were generally quoted recounting their experience that night. The 'other' category within locals does not include Gulf residents, but organizations like local non-profits.

After all of the above categories had been separated, the general local category consisted only of fishermen and residents. It appeared worthwhile to isolate the two in order to detect any similarities or differences in their voices while also outlining the extent to which the coverage prioritizes the voices of fishermen. It also seemed unwise to presume that fishermen have more in common with general residents than with businesses or rig workers. The distinction between business owners and fishermen was occasionally unclear; generally, the quoted fishermen own their own shrimp boats, and thus could also have been considered business owners. There were no clearly identified accounts from a fisherman who worked on a boat owned by someone else, although there were many who were simply identified as 'fishermen' with no further explanation. I continue to refer to fishermen in the masculine only because the articles always do the same, even when discussing the solitary reference to a woman who works on a shrimp boat. This tendency will be discussed in greater detail in the final section of this chapter on voices. 
Politicians and fishermen make up a vast majority of the local voice (see Appendix B). Molotch and Lester (1975) confirmed in their historic study on the Santa Barbara oil spill that officials have a greater voice than locals or conservationists. Most literature on sociology of media suggests that official and authoritative voices generally outweigh all others (Hall et al 1978; Gans 1979, Bennett, Lawrence and Livingston 2007; Molotch and Lester, 1975). The local as I have defined it includes everyone from the Gulf coast. Local is not equivalent to 'general resident' in my sample. Instead, the majority of cited locals have some kind of authority or are explained in reference to their employment on the Gulf. Article 25 quotes two locals, one who is a civil engineer, and another, a retired chemical engineer. I did not classify their voice as a scientific one, since they were speaking as residents of the Gulf. Nonetheless, this is a prestigious job and it is significant that the journalist felt the need to mention their careers at all when they were only speaking as the organizers of a local trout rodeo. This reinforces the importance of 'expert' status in perceived legitimacy as a source, as will be explored in greater detail later in the chapter. The category of general locals also includes a paraphrase attributed to 'a local' with no further information, a group of women who were starting their own cleanup effort, and guest workers from Mexico who lived on the Gulf working at hotels.

Rig workers are quoted 18 times total, but only in three articles. They are only mentioned in articles that discuss the night when the rig sank. Although they were technically BP and Transocean employees, they do not represent their voice as they are giving anecdotal first-hand accounts of the explosion. Rig workers were never quoted in the context of their status as BP employees. Accounts from BP employees were categorized instead as 'BP engineer' or 'BP official' and were organized under the BP 
heading. The prominence of rig workers speaks to a similar trend as that mentioned under fishermen: all quoted rig workers were identified as male. Although their experiences on the rig are doubtless newsworthy, they receive even more overall quotes than all other local businesses or local attorneys.

Politicians have the greatest overall access within the local category with 57 quotes. This trend reflects the oft-cited over-reliance on authoritative sources in sociology of media literature (Hall et al 1978; Gans 1979; Bennett et al 2007; Molotch and Lester 1975). The data demonstrates that politicians were used as a source even more often than fishermen, whose employment was directly impacted by the spill. I chose to include local politicians with the overall local voice, although made sure to tally them as a separate subcategory. I could have amalgamated local politicians in a separate government category, but in many cases the local politicians provided a very different voice than the broader US government. As will be detailed in the section on voices, many Parish Presidents $^{2}$ spoke passionately against BP on behalf of their constituents.

The already diverse voice of locals is further complicated by the presence of powerful, high profile state governors. These voices, such as Hailey Barbour and Rick Perry, often resonate on the national stage. However, separating state from municipal government would also present organizational and methodological barriers. In every other respect I include all residents, politicians and businesses of the Gulf within the local category; to separate governors because they have more power may suggest that I should categorize all voices based on their volume and power rather than on geography, employment or institution. Quotations from these state governors are explored later in the

\footnotetext{
${ }^{2}$ Louisiana is divided into Parishes instead of counties or municipalities and the local heads of government are called Parish Presidents.
} 
chapter when discussing the content of voices. They provide valuable thematic material but do not make up a large portion of the local category.

\section{BP and BP Sources}

The BP category has fewer sub-categories than locals or federal government (see Appendix B). The various people speaking on behalf of BP do not represent the same complexity of voices as locals, or even as the federal government or experts. Locals, government and experts are all categories that contain a range of opinions. On the other hand, BP was represented through a few key people who never swayed from defending the corporation. This trend can also be attributed to my choice to separate BP and anti-BP sources for the reasons discussed above. The company is only divided into two sections: general BP (spokespeople, CEO and managers) and employees (engineers, rig workers and contractors). There was no compelling reason to further narrow the BP category since the speaker was so often a spokesperson that clearly represented the interests of the company.

BP employees are likely to reflect a different voice than spokespeople or the CEO, thus I found it necessary to separate them. The data shows that employees have significantly less say than spokespeople with a total of eight quotes, versus 163 for BP management and spokespeople. Either BP engineers were seldom quoted, or journalists failed to acknowledge that they worked for BP.

\section{Experts}

This category pertains only to scientists, engineers, biologists and academics that were not identified as working for BP or for the federal government. Non-BP engineers could take greater liberties than their BP counterparts in sharing their technical insight 
into the disaster, and were thus worthy of specific attention. Occasionally, the text referred to academics, such as a professor in Political Science. Although authoritative expert sources, these voices also required separate classification and include any voices from the social sciences and humanities, or professors whose discipline is not specified. Academics who work specifically in petroleum research groups seemed especially divergent from a professor in political science, and were likewise categorized separately.

The expert voice (consisting predominantly of scientists and biologists) ranked just below the business and corporate voice in terms of total quotes. Experts are also quoted in $38.6 \%$ of the articles, thus have significant representation. The presence of this category demonstrates the prevalence of expert sources in the news. These voices are presumed to be authoritative and trustworthy by journalists and editors. It may initially appear that scientists, engineers, and biologists could be lumped into a single category. However, the generic term scientist is a particularly vague title for a broad and diverse field. I chose to keep this designation separate in order to see how often journalists used the vague language, or failed to identify the specific scientific field and instead used an institution name, which would require further research on the part of the reader. The use of these terms may indicate how often journalists are generalizing a diverse source group while also reinforcing the authority of the title scientist.

The articles often refer to scientists and biologists, and although they may at times be people who consider themselves environmentalists, they are seldom identified as such. The use of scientist and biologist conveys that the voices are trusted experts and also depoliticizes a potential environmentalist voice. While an environmentalist may discuss systemic issues of responsibility and the politics of blame, classifying this expert as a 
scientist of biologist permits the discussion to be more technical. This seems likely since there are very few occasions that a speaker is explicitly identified as an environmentalist or conservationist, but expert voices do occasionally speak passionately on behalf of the health of the oceans, as will be demonstrated in the analysis of voices in the last section of this chapter.

\section{Environmentalists}

Environmentalists were classified as any person or group that was identified as a part of an environmental or conservationist organization, even if that organization is only concerned with a specific piece of land. At times they overlapped with local non-profits, but generally so long as the group was identified as having a specific environmental affiliation, they were categorized as such.

The environmentalist voice is among the least represented in the sample with 26 quotes in ten articles, only appearing as first quoted three times. This is the second least quoted major category in the sample. It is important to note that this small group is most often represented by organizations like land trusts or conservation groups. and were infrequently identified explicitly as environmentalists or by the names of recognizable environmental groups. Thus, these groups were not always self-evident as a conservation group, and require outside research to confirm their institutional mandate. However, Greenpeace was mentioned in one article in the sample, the only representation from a well-known, national environmentalist group.

\section{BP and Government}


This category emerged because there were ten instances where BP and the federal government were quoted as a single voice. For example, Article 33 reads: "Both government and BP officials said on Sunday that they had no accurate idea of how much oil was spilling into the Gulf." I isolated this category in order to gain a sense of how often journalists quoted the two institutions as a single voice. It seemed unrepresentative to count this category under 'other', or arbitrarily choose to place the ten as BP and the government. In these ten cases it was never clear that either BP or the government was the primary voice or I would have simply classified it as the respective voice it represented. Separating this category rather than amalgamating it with either BP or the government takes away from the totals of $\mathrm{BP}$ and the federal government and by comparison makes the local representation appear higher. However, not only would amalgamating the category be organizationally difficult, but isolating it demonstrates the instances in which the government and BP voices on the spill were so similar that they were reflected as a single opinion. BP and the federal government were never quoted as a single entity without the article featuring other quotes from either BP or the government. Thus, separating these quotes does not significantly affect the "quoted in total number of articles' category.

\section{Officials and Analysts}

Journalists use generic terms like 'officials' and 'analysts' 13 times in lieu of an identified organization or person. I was faced with deciding to what extent the journalist was inferring a general position, or had used an unattributable paraphrase. Often the term 'officials' was used, and in the next paragraph a quote from a BP engineer would appear. In these cases, 'officials' was likely being used to denote BP officials. While discussing 
the static kill procedure, Article 54 repeatedly refers only to engineers rather than $B P$ engineers, even though the context of the article suggests the engineer worked for BP. The prominence of unattributable paraphrases leaves the reader to infer the speaker of the 'official' voice. By suggesting that analysts produced these paraphrases, the journalist assigns authority to a piece of information that cannot be traced to an actual speaker and implies that it is a trusted source.

\subsection{Analysis of the Tables}

This section details the findings that emerged from categorizing, counting, and quantifying the quoted participants. As explained in Chapter Three, there are four tables in the data, all contained within appendices A, B, and C. Table one (Appendix A) lists who was quoted from every category and subcategory in each individual article. At the bottom of Table One there is a separate chart that shows which source was quoted first in each article. Table Two (Appendix B) sums the data from Table One and shows the total quotes for each category and subcategory. Table Three (Appendix C) shows how many articles each category has at least one quote and the percentage that represents. Table Four (Appendix C) totals the first source quoted in each article and the percentage that represents. This section is organized around analysis of the data within these tables. Thus, this section focuses very clearly on access to the news and sourcing patterns.

\section{Table Two: Total Quotations for Federal Government, BP and Locals}

Locals are quoted a total of 198 times, the federal government 188 and BP 171 (Appendix B). As explained in Chapter Three, the category of locals includes any person or business from the Gulf States. The federal government includes the President, regulatory bodies, senators and coast guard, among others. In contrast, BP is a single 
organization. It is significant that BP has nearly equivalent access to the media as the entire federal government. BP has 27 fewer quotes than all of the people, employees, politicians, businesses and attorneys from Texas, Louisiana, Mississippi, Alabama and Florida. If I combine BP with the category of 'business and corporate', this general business voice far outweighs any other category with 248 quotes (Appendix B). As is discussed in the Chapter Three, I chose to count the total quotations by the number of paragraphs in which they appear, never to exceed one per paragraph for a single person or institution. This strategy gave the best sense of the content of the articles. In some feature articles that focused on a specific family or fishing crew, locals were quoted for pages in a row. Article 68 quotes local fishermen for a total of 25 times and their families 5 times (Appendix A). There were no equivalent articles that chronicled a full day as experienced by BP CEO Tony Hayward or President Barack Obama. The high level of local quotes in a single article is significant when considering that BP only has 27 fewer quotes than locals overall (Appendix B). Similarly, Article 26 summarizes the reactions to the spill by different state governors, giving local politicians 15 quotes in a single article (Appendix A). These individual stories dramatically shift the presentation of access as told by total quotes alone. For this reason, it is important for this research to also integrate total articles within which each source category is quoted, as well as who is quoted first in each article.

As previously discussed, literature on the sociology of media suggests that a reader may expect to find institutions and trusted expert sources quoted more often than local residents (Molotch and Lester 1975; Anderson 2002). While my data demonstrates that locals are quoted more often than any single group, this is not an entirely fair 
comparison because all other categories generally represent institutions. Institutions like $\mathrm{BP}$, other businesses and corporations, and the federal government receive the vast majority of access, while only a fraction of access actually goes to ordinary people (refer to the 'general local' category in Appendix B). As well, the totals for BP, the federal government, and locals are all extremely close. The access in these categories appears especially similar when considering feature articles that quote residents or local politicians for many pages. BP is also segregated from other similar corporate voices.

\section{Table Three: Quoted in Number of Articles}

'Quoted in number of articles' tabulates how many articles within which each category has at least one quote. Each major category is counted once for each article within which they appear, regardless of how many times they are quoted in the article itself. This measure helps to account for duplication and the tendency for a single speaker to be quoted for many consecutive paragraphs, or dozens of times in a single article.

The federal government has a voice in 46 of 70 articles, BP in 38 , locals in 34 , business and corporate in 27 , and experts in 27 . In this case, BP and business cannot be combined to get an overall picture of the corporate voice, as they are quoted in many of the same articles. However, when I accounted for duplication, BP and business are quoted in a combined total of 46; the same amount as the government and 12 more than locals. The top three categories are yet again the federal government, BP, and locals, but in this case locals are a close third in representation. This data further reinforces the observation that although locals may have a high number of overall quotes, this can be partially attributed to a high volume of quotes in a few articles that focus on the local experience for human interest and feature stories. Locals have lower access than BP or 
the federal government in stories overall. It is unsurprising that the federal government is highest quoted overall since their voice includes the Coast Guard, who was deferred to as the expert voice in a great number of articles. Any discussion of the spill by the President was also given considerable media attention. In sum, a local voice is featured in fewer articles overall than BP or the government, but is featured in a higher saturation in a few articles. The federal government is most likely to be quoted in any given article, but when combined, BP and corporate voices have equivalent access to the government. This finding further indicates that journalists rely on authoritative sources when constructing stories.

\section{First Quoted: Table Four}

First quoted is an important measure in order to give context. Although some groups may often have a quote or two as token representation toward the end of an article, the first quoted indicates how often a particular group is cited at the beginning of an article, and likely as the trusted first voice. Hard news stories are often presented in an inverted pyramid format, which presents the information deemed relevant first, and works down to the details (Bell 1995). The widespread use of the inverted pyramid is explained by the journalistic assumption that a large portion of readers do not read to the end of articles. However, the formatting itself may cause interest to wane as readers progress through the article. The prevalence of this format makes the first quoted especially relevant to study.

BP and the federal government each occurred as the first quoted in 20 articles, while locals were quoted first in 13. Again, it is significant that BP and the government have identical access to the first quotation, each occurring first in almost $30 \%$ of the total 
stories. BP is a single organization, and it's $30 \%$ does not include other oil companies. Conversely, the federal government includes a broad array of institutions and actors. BP is quoted in slightly fewer articles than the federal government and less often within them. This indicates that BP has higher access than other sources to the first quote of the article.

Although locals may appear in a large portion of the overall articles, (73\%, surpassed only by the federal government) they were often quoted later in articles, which is reflected by the dominance of $\mathrm{BP}$ and the federal government as first quoted. Locals may often be included later in an article for balance, but they are much less often trusted to be the first source for an article. All other categories have consistently low access to being first quoted when compared to locals, BP, and the federal government. Although many of these voices have low representation in total quotes as well, they have even lower access to being first quoted.

\subsection{The Voices}

The previous section provided an overview of access afforded to the different source categories in the sample. Some preliminary measures were used in order to understand how the quantity of access was organized. The current section adds to this discussion by considering the content of these quotes and the construction of different source voices. The use of the tables in the appendices could only provide a preliminary assessment of the article content. For example, I could not numerically tabulate the subtle ways that various sources assigned blame for the spill or posited their version of moving forward. 
The general presentation and construction of key source groups is crucial to the overall project. My use of frames is rooted in sources who promote various frames. Frames are linked to different ideological versions of how a society should respond to industrial environmental accidents. I must take the time to understand the construction of sources by the journalists who design the news. Journalists rely on sources due in part to the objectivity paradigm, as was discussed in Chapter Two. Thus, sources shape and are shaped by the news. An examination of how each source group was portrayed sets the tone for the examination and extraction of frames.

\section{Locals}

Beginning from the standpoint of sociology of news, one may expect the local voice to be marginalized or excluded. As was detailed in Chapter Two, Anderson (2002) specifically notes that media discourse on oil spills demonstrates over-reliance on official sources. Carlson (2010) echoes her sentiment by explaining that common to most media studies is a finding that official sources are relied upon by journalists as trusted sources. As discussed earlier in the chapter, Molotch and Lester (1975) agree that official sources have greater voice in media than locals or conservationists. However, they also suggest that openings in the status quo of journalism may be provided by a sudden and unexpected event like an oil spill, which was demonstrated in their study by the voices of conservationists and locals. They argued that oil spills are sudden and unexpected, thus become events accidentally. Temporarily, these events can thus create space in routine coverage for the voices of conservationists or dissenting locals. However, in their study, this increased access was limited to the local Santa Barbara area and depended crucially on the political economic context of the spill site. 
In my sample, locals have a relatively high representation, but this representation is tempered by a number of constraining factors. More significant than how often locals are quoted, is the content of their quotations. Far from a scathing criticism of the oil industry, the local voice bore a striking resemblance to the rhetoric of the federal government, whose total quotations are a close second to locals and who have access to the largest portion of the articles. Many local voices echo concerns about sustaining the oil industry and local jobs. Substantively their voice lacks a far-reaching criticism of BP, and is often used only as a human-interest piece rather than delving into the causes and consequences of the spill on a large scale. These themes will be elaborated upon in the section to follow.

\section{Simplified Human-Interest Piece}

As discussed in Chapter Two, there is a broad spectrum of reasons that may lead the products of the media to be individualized and simplified. This includes neoliberal narratives, which downplay the social repercussions of problems. News may also be simplified due to constraints on the media such as reduced budgets, the 24-hour news cycle, and a procedural focus on events rather than issues (Anderson 2002, Hannigan 2006). These various conditions lead to a media environment that has been observed by a number of theorists to 'personalize' the news. Jewkes (2004) suggests that stories may be increasingly presented as human-interest pieces that present political and economic issues as conflicts between different people. Various theorists writing about the news have talked about "personification" (Ericson Baranek and Chan 1987; Hall et al 1978). These authors explain that the broader narrative of an event may be reduced to a story from a select few individuals. This can have the effect of depoliticizing a story since the focus is 
then placed on those individuals to the neglect of systemic issues. Although the local voice in this sample may at times be abundant, the stories often detail the day of a family on the Gulf, or the sorrows of residents who derive their livelihood and heritage from the water; these stories are characteristic of human-interest pieces that focus on the specifics of a family or person. Although these pieces are doubtless important, the only history or long-term narrative evoked is that of the life-giving Gulf and the dependence of locals on its water as a way of life.

The locals are presented as unlucky but resilient people and their stories give a human voice to a technical disaster but provide little in terms of content. There are a small number of stories where the locals are deeply disappointed and skeptical of the accounts provided by both the government and BP, but they do not engage in conversation about blame beyond the assertion that BP must be held accountable.

Occasionally, the finger is pointed at a lack of government regulation but there are still no broader conservationist frames advanced by the featured local sources; no voice suggests that Americans may need to reduce their dependence on oil or that deepwater drilling is unnecessarily risky. Despite their apparent sorrow at the destruction of the Gulf, none of the locals are presented as questioning the economic and political system that led to the spill.

Locals are romanticized as simple people who possess folk knowledge about the Gulf. Although locals often express anxiety at their economic insecurity, the stories focus on their deep love of the Gulf; locals prioritize a way of life over a job or paycheque. Specifically, the portrayal of black oystermen demonstrates that the Gulf people are shown as simple, hard working and down on their luck (see box IV.1). This passage 
romanticizes the locals as hard workers with fishing in their blood, implying that life and work on the Gulf is ingrained in the biology of its people.

[Article 47: Way down in the delta, just south of the Belle Chasse Ferry at Beshel's Marina here, black men with work-worn hands and several generations of fishing in their blood sat around on old milk crates, hoping for a piece of the oil cleanup action that seems to have bypassed their little stretch of the bayou.] Box IV.1

This article focuses on lost livelihood, and not so much on actual lost revenue or a dollar value. The locals discuss the impaired capacity to raise a family through the hardworking lifestyle to which they are accustomed (see box IV.2).

[47: "You might see a time when there ain't no more black fishermen around here," said Warren Duplessis, 49, a deckhand for a two-man oystering operation. "Because now you can't raise no children off the side of a boat. Nowadays you've got to take him out of here, let him learn something with the books. No future in what we've been bleeding and sweating for all our lives."] IV.2

This passage includes slang and the use of a double negative, "can't raise no children..." Although many of the fishermen are portrayed this way, the representation of black oystermen directly reinforces the stereotype of Gulf residents as simple, uneducated people trying to survive on the trade that their fathers have been doing for generations. This voice contrasts sharply with expert voices, who are instead given authority and who are not portrayed speaking in regional dialect or making grammatical errors. Although this article seeks to bring attention to the issues of race in BPs hiring practices, it reproduces racial stereotypes and presents the black oystermen as simplistic and unconcerned with the complicated reasons the accident occurred.

[47: He shook his head and took a long, deep gulp of the beer in his hand. "We just have to tough it out," he said. "I know I'm going to make it. I know it like I know them waters out there. I'm going to make it."] IV.3

The quote in box IV.3 also portrays the Gulf residents (specifically the black oystermen) as resilient and confident that they will persevere. The passage implies a that 
locals possess folk knowledge of the Gulf waters. The locals in this article are never shown discussing why they believe the well exploded. who is at fault, or what repercussions should come from the spill. They instead represent an antiquated myth of the hard working American, down on their luck but clinging to the belief that they will overcome their circumstances.

The journalists chronicle the lives of locals for in-depth feature stories, like Article 68. It details a hardworking fisherman father and his conflict with his son, who wants to be a shrimper. The author suggests that the spill complicated his dreams in an already difficult industry (see box IV.4).

[Like any young man approaching adulthood, he clashed with his father even as he sought his help. And the day he grew frustrated over a sheet of fiberglass would not be the last that he had the impulse to bolt.

That evening, Buddy, 43, watched with thick arms crossed as Aaron packed a duffel bag, told his mother goodbye, and peeled out of the yard in his old Mustang.

Their son, Buddy complained to his wife, cared too much about his fast cars and fancy sneakers.

"He has to learn, Carolyn," Buddy said. "He wants to do this, he has to learn it ain't all peaches and cream." ] IV.4

Although this article stays predominantly at the surface level and encapsulates life on the Gulf in a single family, it reads like a stereotype of hard working, family-oriented Americans and uses the shrimper as an example of a remaining vestige of the entrepreneurial spirit in the American dream. The story fails to encapsulate their struggle as that experienced by so many people under a system that has eroded domestic labor and quality of work, but instead focuses on the fiery spirit of the son who wants to be self employed on the water. This article chronicles the life of the young man, including his Facebook updates to his girlfriend, who he met as a consequence of the spill and believes is the only good thing to have come of it. 
In sum, locals predominantly provide anecdotes and do not have a real voice in a discussion on the causes and consequences of the spill. The only extended conversation with locals comes in the form of feature stories where the journalist portrays them as topics of human interest. This personification may further obscure the reality on the Gulf because these stories are narrowly constructed as individual stories of experiential knowledge while reinforcing stereotypes and personifying average, hardworking Americans as simple people whose only desire was to preserve the Gulf on which they survive.

\section{Locals as Emotional}

The local response is represented as one that is highly emotional but fails to engage with any complicated issues or analysis. This point is intimately connected to the previous discussion on how locals are simplified, and focuses specifically on emotional responses as a mechanism of simplification. The local voice generally laments their loss rather than discussing the circumstances that led to the situation on the Gulf. Local anger toward BP is infrequently portrayed. The passage in box IV.5 generalizes locals as grieving.

[Article 30: And in interviews across southern Louisiana last week, the responses included anger, denial and naked grief.

"A lot of times I want to go stand in a corner and cry - not so much for me, because I've done it a long time, but for him," said Mr. Greco, 43, nodding at Aaron as they stood in line at Kentucky Fried Chicken during a lunch break from their training classes on Thursday.] IV.5

The father laments loss of the lifestyle and livelihood that he experienced, and wishes the same could have been preserved for his son.

The local voice is represented as simplistic and unsophisticated. Box IV.6 shows a local who conceptualizes the spill as a basic battle against an antagonist in a film. His 
unnamed 'bad guy' is presumably the oil, since there have been no illusions of killing the company BP.

[Article 29: "It's like a bad movie that just won't end," said Billy Altman, 45, a mechanic in New Orleans. "You know, you think they finally killed the bad guy, and then he comes back to life. It's crazy." ] IV.6

The bad guy is presented as the spill rather than the organizations that caused the spill to occur. This local does not explain where the bad guy came from or how to get rid of him, only that he wants him dead. This tendency contributes to a theme explored in the following chapter on frames, where the oil spill is portrayed as a causeless force of nature rather than an event that arose out of very specific circumstances. A local is distressed when talking about the spill, and compares it to the hurricanes he has endured: "He choked up when asked about the spill's effect on the lives of his neighbors. 'It's unspeakable,' Mr. Mitchell said. 'I've been through five hurricanes and now this'" (Article 35). Another local, cited in Article 30, describes his helplessness and disgust: "It makes you want to throw up when you see it because you know it's coming and you can't do anything about it." This local feels there is nothing he can do and does not shift the focus to those who can do something, but resigns himself to the catastrophe approaching the shore.

As the articles progress to the conclusion of the spill, Article 51 reflects local anxieties about the cleanup: "More than a third report children with new rashes or breathing problems, or who are nervous, fearful or 'very sad' since the spill began. And even though the gusher of oil has been stanched, almost a quarter of residents still fear that they will have to move." The article reflects deep insecurities among Gulf residents, 
and suggests again that they are emotionally distraught by the spill. Thus, the residents are portrayed as anxious and sad. but the reasons for these feelings go unexplored.

Another passage from Article 68, which details the lives of the Grecos, leaves grammatical errors and shows the rage of local residents (see box IV.7).

[68: And when the oil company's efforts to cap its leaking well fell short, Aaron recorded it on his Facebook wall. "BP fails.... AGAIN!!!" he posted on May 29. Then, on June 15: "Sleepless night, lots of thinkin goin on."] IV.7

In this segment, Aaron expresses frustration and anger, which he directs at BPs failure to cap the well. The journalist chronicles the details of Aaron's day, even his facebook posts and eventually includes messages between Aaron and his girlfriend.

One of the more critical local voices may be an example that is not a person at all. Article 22 cites a local holding a sign for President Obama's motorcade that reads: "Clean up the Gulf!" in dripping black paint like oil. Despite being more critical than many others, this voice was actually silent, and restricted to the boundaries of a sign. This local is making a powerful statement to the leader of the nation, but still only proposes that the Gulf be cleaned. Directing energies at cleaning up the Gulf is a logical response since the work and health of locals are in jeopardy, but this example illustrates a failure to provide local access beyond cleanup or to engage them in a conversation about the bigger picture.

\section{Locals as Unlucky but Resilient}

As has been briefly discussed, the Gulf residents are often portrayed as resilient, hardworking Americans who refuse to let the spill ruin their quality of life. The New York Times coverage often cited the spirit of the local people as being a rare variety that could survive a tragedy. The articles often praise the character of Gulf residents as 
unique. This framing allows readers from the rest of the country to relax in the faith that once the well is capped and the Gulf cleaned up, this accident will not tarnish or permanently damage the residents of the Gulf.

One quotation shares the voice of a local woman who does not work for the fishing industry. Rather than using her unique viewpoint as a female or as an educator, she is given brief access and suggests that New Orleans is the last city to deserve the spill: "In New Orleans, Margaret Shockey, 67, a retired teacher, said, "One thing's for sure, this is the last city that deserved this" (Article 29). Margaret believes that New Orleans is a particularly unlucky city. In suggesting that the oil spill is a consequence of bad luck, similar to hurricanes, she ignores the failings of a specific company, industry and regulatory structure. This trend is connected to a framing practice outlined in Chapter Five that treats the Gulf spill as a natural disaster rather than a man-made event. This framing practice naturalizes and depoliticizes the spill.

The residents of the Gulf are praised not only as embodying the American spirit, but also for being passionate and outspoken: "Asked what he would have done if the mayor had been arrested for such a move, Mr. Jindal sounded like the kind of populist warrior that Louisiana has been particularly fertile in producing" (Article 22). This article demonstrates a taken-for-granted assumption that Louisiana creates populist warriors; that the people are fiery and passionate and will not be beaten down by bad luck. This journalist assumes that there is something unique in being raised on the Gulf coast, which generates people who are willing to fight for one another in order to preserve their way of life. 
Again, the independent spirit of fishermen is noted as being unique, presumably because they chose a life of danger and isolation on the water (see box IV.8).

[There was talk of a "farm bill" for shrimpers that would protect them under the same kind of social contract that had subsidized America's family farmers for decades. But the vaunted independent spirit of commercial fishermen made them hard to organize, and without assets like land and equipment, they had little political influence. Article 68] IV.8

The article subtly suggests that the fishing industry is suffering under the weight of the same economic system that led to the blowout in the first place. A local in Article 68 explains that shrimp is worth less money now than it was in 1990, and the journalist explains this has to do with low-quality farmed shrimp from China. The American appetite quickly moved from predominantly domestic to imported seafood, but there is no conversation around why that change occurred. The articles instead focus quickly back on the resilient character of people from the Gulf. Specifically, the article points out that the unique character of independent fishermen makes them difficult to organize, rather than looking to a steady and politically motivated decrease of organized labour and social protections in the United States.

\section{Local Politicians}

Although local politicians are included in the same broad category as residents and fishermen, it is a mistake to presume this voice is homogenous or rallies in any unified way against BP. Although many local politicians were given similar voices as locals (straightforward, angry, sad and fed up with BP), there are certain state governors who spoke out loudly on behalf of business. Article 26 explains that Governor Rick Perry of Texas has long stood up against "onerous" environmental regulations. He called the spill "an act of god" two weeks after the explosion. Governor Haley Barbour of Mississippi criticized the tendency to overreact rather than critiquing BP or the oil 
industry. He was quoted saying: "A bunch of liberal elites were hoping this would be the Three Mile Island of offshore drilling." He accused the news media of exaggerating the disaster, described the oil on the Gulf as "very, very, very thin", and dismissed comparisons to the Exxon Valdez. These are not the typical voices of local politicians: they are high profile, powerful state governors who speak out blatantly in favor of business to the neglect of environment.

There are other state governors who do not speak out on behalf of business. Florida Governor Crist suggests an outright ban on offshore drilling (Article 26). He frames the spill in a more structural way than most environmentalists in the sample. Florida stands to lose more from an oil spill than any other state because it collects no revenue from oil and gas, and relies almost exclusively on tourism. It would be unrepresentative to suggest that positions of higher power always correlate with support for the oil industry. Instead, the interests of state Governors often correlate with the business interests and political economic history of the particular state. The implications of these passages will be explored in greater detail when looking at the themes in the following chapter.

Other local officials launched ambitious publicity campaigns against the damaging effects of the spill. Governor of Louisiana, Bobby Jindal, went forward with his own clean up procedures without the permission of the Coast Guard. He passionately campaigned on behalf of his constituents, but often sounded as if he was campaigning against the spilling oil itself rather than any of the forces that led to its release. Jindal encapsulates the high quantity, low content response typical of the federal government and many locals; he spoke passionately about his own rogue plans to clean the spill, but 
failed to conceptualize blame as something more complicated than public shaming of BP. Jindal suggests that the only relevant facts to the spill are those "on the ground" (see box IV.9). He casts aside discussion and moves forward to the immediate physical circumstances and response.

[Article 26: Mr. Jindal, a Republican with national ambition, is not going to leave room for anyone to say that he was not vigorously engaged, did not appreciate the threat or did not know the facts - every single fact - on the ground. Almost daily he tours the coast by helicopter or boat, then returns to land and holds a news conference, surrounded by angry mayors and parish officials who take turns describing the inadequacy of the response effort.] IV.9

The passage in the box suggests that the best spill response would be vigorous, on the ground, and constant. However, Jindal's daily helicopter tours have a high carbon footprint for an observer with no scientific or engineering background. Utilizing his 'on the ground' knowledge, Jindal crafted his own plan to flood delicate ecosystems and estuaries with Mississippi river water, a plan that temporarily kept the oil at bay but destroyed oyster stocks.

Similarly, local Governor Billy Nungesser frames the problem as one with a simple enemy to beat and uses a conflict metaphor to rouse the previously discussed Gulf spirit (see box IV.10).

[Article 32: "I know it's going to be rough," he said to the crowd in a speech that sounded at times like a locker room pep talk. "I know everything's not going to go our way. But they're not going to beat us."

"Go get 'em, Billy," someone shouted from the bleachers. ] IV.10

This passage shows a local responding to the governor's rousing address and simplistic message; the local believes that Mr. Nungesser will fight the oiled enemy on behalf of the Gulf people. In Article 69 Nungesser is documented losing his patience with the cleanup and publicly curses out the coast guard commander. Many have argued that some local 
government responses demonstrate political appetites for votes rather than concerns about a real positive effect on the health of the Gulf or its residents. Political ambitions aside, Mr. Jindal and Mr. Nungesser ask for results, not answers.

Although fiery and independent, local politicians are also depicted sharing in the grief felt by local residents: "Michel Claudet, the president of Terrebonne Parish, 60 miles southwest of New Orleans, said that when he heard the news, he felt "sorrow, despair and like this ordeal will never finish. If you go around the parish, it is all our folks talk about" (Article 29.) He describes the local reaction as emotional. Blame is not discussed; he just expresses the locals' desires to have the ordeal over. This quotation mirrors the portrayal of locals as devastated but lacking a focus for action or blame. Locals reflecting economic lens

In Chapter Five the tendency to prioritize only the economy will be discussed in greater detail. However, there is a noteworthy tendency within the local sample to reproduce this narrative, which lends insight into the construction of their voice. The locals reproduce this frame even though they arguably have the most to lose in the disaster. Republicans, who often tout an explicitly pro-business ideology by suggesting that oil production should never be interrupted for fear of jobs lost, represent four of the Gulf States.

[Although lives were lost in Hurricane Katrina, Captain Dave, 41, believes that his livelihood may be gone forever.

"I'd rather have another hurricane than this," he said as he motored past the oyster beds - now all closed - places he has known since childhood.

He stopped by an inland marsh to examine the grass at the edge of the water, which has turned a murky brown. "With a hurricane, you know when it hits and what the damage is and what you've got to fix," he said. "With this spill, the only answer is to wait."] IV.11 
The passage in box IV.11 cites a relatively high-status, business owning local whose fleet of boats sat unused at the marina during the spill. He suggests that the spill is worse than the hurricane; presumably this captain and business owner did not lose any family or friends in Katrina. Captain Dave prioritizes the economy by implying that there is a nearly indecipherable difference between losing financial livelihood and losing lives. In contrast, the black oystermen discussed previously, are presumably of significantly lower economic means than Captain Dave. They mourned their way of life and feared for the futures of their children. but never suggested that the spill could be worse than the storm. The passage in IV.11 also reflects the psychological stress associated with a drawn out disaster that has no clear solutions. However, Katrina arguably also felt endless to all of the displaced people who lost loved ones, their homes, schools, and local hospitals, many who still live in temporary housing.

There is a focus by many quoted fishermen on lost revenue and the future of the fisheries (see box IV.12).

[Even if the seafood of the Gulf is deemed safe by the authorities, resistance to buying it may linger among the public, an uncertainty that defies measurement and is on the minds of residents along the entire Gulf Coast.

"How do we get people to buy our food again?" Mr. Johnson asked.] IV.12

This passage suggests that Gulf residents have an economic focus; after the spill is cleaned up, they will worry about public perceptions of unsafe Gulf seafood. Rather than sharing a concern for the legitimacy of claims that the food is safe, the fishermen are concerned with restoring demand.

It would be unfair to equate a local framing of the disaster as a financial concern with the industry frame inherent in some corporate and government voices; locals require financial prosperity for their day-to-day lives, a concept frequently evoked by politicians. 
Fishermen may stand to lose the most in the destruction of the Gulf as they derive their livelihood directly from the water. However. the under representation of any locals who do not work in the fishing industry indicates that the spill may be framed predominantly as a problem to industry and work, and reflects a neoliberal focus on business and financial concerns. This neglects any discussion around the general health and quality of life for Gulf residents who do not directly extract their livelihood from the water or from industries that depend intimately on the coast.

\section{Omitted local voices: Women}

The articles contain a noticeable absence of female voices. There is over representation of men in nearly every professional category including politicians, biologists, and CEOs. The one category where one might expect women to have higher access is within the locals, but preference is strongly to cite fishermen. Only one woman who worked in the fisheries was mentioned, and she was still referred to as a fisherman (see text box IV.13).

[Another survey respondent, Sherry Mareno, 39, a Buras, La., fisherman who lost her job after the spill, is trying to sell her house and leave the Gulf Coast. She and her 11-yearold son both developed serious skin rashes, and she blames the chemical dispersants used to fight the oil. ] IV.13

Although there are a few references to the ability to raise a family in the sample, it is significant that the lone female voice working in the fisheries is described worrying about the health of her son rather than discussing her lost haul or life on the bayou. This passage reinforces stereotypes about the prominence of the family and household to women's lives, rather than orienting them within the broader sphere of labour, as is often done for men. Through choosing to orient the local conversation around fishermen and their labour, the journalists explicitly focus on the masculine in the title, and 
inadvertently prioritize male voices by focusing coverage on a male-dominated industry. One article cited Mexican guest workers, who were also all male.

\section{Environmentalists}

The environmentalist voice echoes many of the aforementioned trends. Their access is limited, and within the space they are allotted, they often focus on the technical details of the cleanup or even blame the cleanup workers. In Article 31, environmentalists are discussed in a general sense as being distraught at the way cleanup crews trampled delicate marsh grasses. The concerns expressed by the environmentalist in the article was only on the micro level and discussed how marsh clean up was being mishandled by individual workers. The article referred to the employees as flawed "zealous cleanup folks" rather than looking to their management, training or instructions. The environmentalists raise suspicions that the cleanup crews are focused on giving the appearance that they have cleaned rather than actually avoiding damaging the ecosystem further. Thus, these voices imply that the crews are lazy or unmotivated rather than poorly trained or carrying out poor instructions.

Greenpeace is quoted in Article 31. Their single quote reads: "The larger reason for these efforts seems to be to make it seem that they are doing everything they can, when tragically, there isn't much that can be done to clean up a spill of this size and nature." This quote directly follows a paragraph explaining that environmentalists believe the cleanup crews are only concerned with giving the appearance of cleaning. Although if placed back into context of the full interview, the Greenpeace spokesperson may have been referring to BP's mandate, the placement of the quote implies that the spokesperson is directly discussing the cleanup workers. The Greenpeace quotation also frames the 
spill as a tragic accident and fails to discuss broader reasons for the event. The discussion is focused on the impacts on wildlife, but only in the short term. For example, a toxicologist is relieved to know that nesting season will be over soon, but does not discuss what impact the spill itself may have on future nesting.

The environmentalist voice is often represented as informative but simplistic. They focus on technical, scientific detail, similar to biologists: "I would look at your filter feeders first, and then your crabs and your shrimp, on up the food chain to coastal fishes" (Article 11). This voice is scientific and dispassionately discusses the technical process by which the oil will affect marine life. Although it may be classified as an environmentalist quote, the content demonstrates efforts to be apolitical and objective.

One article explains that politicians are ignoring environmentalist groups (see box IV.14).

[Article 26: Casi Callaway, the executive director of Mobile Baykeeper, an environmental group, said she could have told him that would happen - if she and others with coastal expertise had been consulted. But they have had a difficult time getting through to the decision makers, she said.

"For presence, [governor Nussenger] gets a 100," Ms. Callaway said. "He's flying the area and saying, 'We need protection there,' and pointing, and it's happening to a large degree. But it's not always the areas that are more visible that are more meaningful to protect."] IV.14

This quotation demonstrates that the responses from state governors may often be an effort to make political gains. This environmentalist voice criticizes Nussenger's display; she commends his visibility, but suggests that he is ignoring the advice of those who best understand the coast. This single quote encapsulates the entire environmentalist voice in the article and she does not go on to discuss what a proper response would look like.

An environmentalist in Article 28 suggests that Americans have faith that technology will solve their problems, which was proven to be misguided in the case of 
the BP spill. The cited environmentalist speaks to this specific example and does not elaborate on the implications of a failed social belief in technological progress.

Once the oil stopped flowing, environmentalists were cited downplaying the effects of the spill (box IV.15).

[Some of the compounds in the oil evaporate, reducing their impact on the environment. Jeffrey W. Short, a former government scientist who studied oil spills and now works for the environmental advocacy group Oceana, said that as much as 40 percent of the oil in the Gulf might have simply evaporated once it reached the surface. ] IV.15

Shortly after the spill was capped, a wide range of voices suggested that the oil was disappearing faster than anyone expected. The environmentalist in box IV.14 was used to further validate that point and provide technical scientific basis for the quick disappearance of the oil.

\section{Experts}

Expert voices are generally portrayed as apolitical and technical with a few notable exceptions. Chris Reddy, director of the Coast Ocean Institute at the Woods Hole Oceanographic Institution, conveys his trust in officials and their spill response. He states that he is impressed with the response to the spill thus far and believes that Americans are in good hands (Article 4). He thus implicitly gives credit to BP, the Coast Guard, and the government, while implying that the public should trust in these forces as well. In this case, the expert supports the institutions responsible for the spill. In most instances the expert voice is used to explain the technical details of the event. Experts were cited very often after the spill was plugged, and suggested that there are oil-eating microbes in the Gulf that could account for the disappearance of the oil, as discussed above. Although there are exceptions, this voice is often removed from the political and the long-term.

Conversely, there are experts who represent the opposite frame (box IV.16). 
[Article 38: But some believe that studies on the impact of oil and dispersants should have been done long ago, given the proliferation of drilling rigs in the Gulf of Mexico. "Some of these studies were proposed years ago, and the agencies decided not to fund them," Dr. Ross said. "We're paying the price for it now." ] IV.16

This quotation shows an example of a scientist representing a somewhat environmentalist frame. The scientist suggests that the effects of oil and dispersant should have been studied long ago. By 'the agencies' the implication is that he means federal agencies, and thus that the government did not adequately invest in the potential repercussions for an oil spill in the Gulf. Although this discussion is brief, it implies that there is at least a somewhat structural problem with funding and priorities.

Other experts have also reflected a long-term concern (see text box IV.17).

["We've never had a spill of this magnitude in the deep ocean," said Ian R. MacDonald, a professor of oceanography at Florida State University. "These things reverberate through the ecosystem," he said. "It is an ecological echo chamber, and I think we'll be hearing the echoes of this, ecologically, for the rest of my life." ] IV.17

This passage reflects grave long-term concern for ecosystem health. This biologist suggests there will be long-term environmental consequences. Rather than taking on the apolitical and technical expert voice that is often demonstrated in the sample, this biologist demonstrates environmentalist concerns. His discourse more closely mirrors the environmentalist talk that I observed in alternative media than many of the environmentalist voices in the sample.

\section{BP}

BP's response changed dramatically through the course of the spill, and often focused on trying to stabilize their public relations after Tony Hayward made several damning public comments. BP often ignored the causes for the accident, claimed they were not responsible, blamed other parties, and eventually accepted responsibility, while 
officially blaming other corporations. As demonstrated in Article 5, BP explicitly denied responsibility for the disaster but said they would nonetheless clean it up. In this early stage, they failed to reassign blame to another guilty party. This was the first instance in which BP presented their corporation as heroic: a corporation who is not to blame for the accident, but will nonetheless commit all their energies to stopping it and cleaning it up. BP often decontextualizes the spill and implies that the event is causeless; their general frame suggested that the spill was an unfortunate and unlikely accident, as will be explored further when looking at their framing practices in Chapter Five. Hayward said: "This is like the Normandy landing, we know we are going to win. We just don't know how quickly" (Article 13). Hayward demonstrated unwarranted optimism since there were no successful technologies thus far, nor had a well at that depth ever been capped. Hayward also suggested that there would be a winning outcome from an oil spill that had already progressed for weeks. Although it is not as simple as a winning or losing outcome, the situation was arguably much closer to having already lost, leaving damages that are likely irreversible. The spill is often framed with a battle metaphor, and in this case, Hayward explicitly references a famous battle in conventional Western war history. This strategy channels American patriotism and casts BP as heroic by aligning the company with the Allies, who are the suggested protagonists of this battle.

After the first capping procedure failed, Hayward again touts BP as charitable: "'I am disappointed that this operation did not work,' Tony Hayward, chief executive of BP, said in a statement. 'We remain committed to doing everything we can to make this situation right"" (Article 13). Again, Hayward presents the spill as a situation without causes or blame, and praises BP's willingness to make it right. However, the capacity for 
the spill to be made 'right' is debatable. Eventually, BP accepts responsibility. Article 23 reads: " $\mathrm{BP}$, the oil company responsible for the spill, has pledged $\$ 500$ million for research." However, BP accepts responsibility as a duty to clean up the Gulf, not to be confused with accepting blame, which they maintained should be directed toward Transocean or Halliburton. As well, the use of 'pledged' implies that BP is making generous contributions to the research community. BP repeatedly denied they had been negligent, but nonetheless continued to ramp up their spill fund (Article 44).

At one point relatively early in the progression of the spill, Tony Hayward had an exchange with a fisherman (see box IV.18).

[Article 13: He finished the day meeting with officials in Venice, La., where he spoke to fishermen loading booms on boats, asked for advice, and thanked them for their efforts. "You guys are doing the best you can," one fisherman said.

Mr. Hayward replied: "We're trying very hard. If we could do more, let us know."] IV.18

When meeting with fishermen Hayward very likely heard significant negative feedback on the spill. However, the solitary quote in box IV.18 shows a fisherman who empathizes with BP. It shows camaraderie between the CEO and the fisherman while praising BPs efforts as valiant and commendable. Early in the spill many voices were depicted empathizing with BP.

Eventually Tony Hayward was dismissed from the company (see box IV.19).

[Article 42: Mr. Hayward said his departure would allow BP to repair its reputation. "It's a very sad day personally," he said. "I love this company and everything that it stands for. For it to move on, particularly in the U.S., it needs a new leadership."] IV.19

Hayward suggests that he is making an honourable personal sacrifice for the good of the company. Article 42 also suggests that this move was made for the good of the U.S. rather than for the good of BPs public relations in the United States. The talk again commends $\mathrm{BP}$ for prioritizing the American people and making tough decisions. 
Tony Hayward also made statements that were seen as insulting to Americans

(see box IV.20).

[Article 19 discusses the claims process by people whose income is impacted by the spill: "BP is. obviously, interested in where that money goes, hence the need for adjusters on the large claims and the comment by Tony Hayward, the company's chief executive, to The Times of London: 'This is America - come on. We're going to have lots of illegitimate claims."'] [V.20

The paternalistic sentiment seems to be that despite their status as the self-proclaimed 'responsible party', BP is the moral authority on the legitimacy of claims. British Hayward is stereotyping Americans as the tabloids reflect them, quick to sue and always looking for an avenue to easy income. It is bold of BP to judge the legitimacy of claimants when many claimants would judge the legitimacy of their drilling practices. BP is the guilty party, but frames the potential claimants as guilty without any evidence provided of illegitimate or fraudulent claims.

Occasionally the articles cite BP material from before the spill. Article 28 cites a 2005 speech by then president of BP's Gulf operations (see box IV.21).

[Article 28: In his 2005 talk, Mr. Eyton, now BP's group head of research and technology, was not so cavalier, discussing the need for vigilant risk management. "We find ourselves designing floating systems for 10,000 feet of water depth before the lessons of working in 6,000 feet have been fully identified," he said.

He sang the benefits of technology while acknowledging its danger, expressing hope that fail-safe features and computer modeling could decrease the risk: "We know the premium associated with hardware reliability is high, but at this stage, operators still have a limited failure database for forecasting the required levels of intervention in ever-deeper and more remote environments."

Technology, he added, "becomes both an enabler, while at the same time being itself a source of risk."] IV.21

This segment is atypical of the discourse presented by BP, likely because it was not produced in response to the spill, but five years earlier. Mr. Eyton admits the technological sophistication for drilling has moved forward very quickly. He frames the 
discussion in a common form; around risk management rather than risk reduction, or harm reduction. He is less optimistic than many voices within the industry and he frames the deepwater drilling practices as inherently risky. He suggests that enthusiastic use of technologies to drill deeper should be tempered with caution. He also suggests that unbridled enthusiasm about technology can be a source of danger.

\section{Federal Government}

The federal government always had a voice in the media coverage, but it shifted from primarily through the Coast Guard, to a visible effort by the Obama administration to demonstrate leadership (see box IV.22).

[Article 33: "This is obviously a difficult situation," Ms. Browner said on NBC's "Meet the Press" on Sunday, "but it's important for people to understand that from the beginning, the government has been in charge."

"We have been directing BP to take important steps," including the drilling of a second relief well, she added. ] IV.22

This passage reflects the federal government's efforts to appear in of the broader spill and of BP's actions. As the spill progressed many media outlets critiqued President Obama's failure to show emotion and give leadership, eventually leading to President Obama showing clear frustration with the company. The federal government had a vested interest in minimizing the effects of the spill, but nonetheless voices like those in box IV.23 from the National Oceanic Atmospheric Administration were still given coverage. The quote is cautious and warns against potential long-term impacts on the ecosystem.

["Less oil on the surface does not mean that there isn't oil beneath the surface, however, or that our beaches and marshes are not still at risk," Jane Lubchenco, administrator of the National Oceanic and Atmospheric Association, said in a briefing on Tuesday. "We are extremely concerned about the short-term and long-term impacts to the Gulf ecosystem."] IV.23 
This atypical passage reflects an interest in short term and long-term impacts; this broad response was seldom echoed through press releases from the rest of the federal government. After the spill was capped, discussion of the long-term impacts on aquatic life almost literally vanished from any federal agency and focused instead on how quickly the Gulf had been cleaned and suggested that the seafood was safe. This culminated with images of President Obama swimming in the Gulf with his daughter.

\section{President Obama}

President Barack Obama was heavily criticized during the early days of the spill for being too hands off and not doing enough with the cleanup, especially given the hardship many of these same people had endured after Katrina due to the failure of the government response. Criticisms of his response are discussed at greater length in Chapter Five. President Obama's stance has essentially been a reaction to the heavy criticisms lodged against him where he attempts to empathize with the Gulf residents. His voice prominently entered the sample in range three: "You are not alone, and you will not be abandoned," he said. "We are on your side, and we will see this through" (Article 21). President Obama attempts to forge a connection with readers while reassuring residents that the Gulf spill will not mirror Katrina in its federal response. President Obama tries to demonstrate forceful, trustworthy leadership (see box IV.24).

[Calling the spill an "assault on our shores, on our people, on the regional economy," Mr. Obama acknowledged that the recovery effort would be long lasting and pervasive.

"This isn't just a mess that we have to mop up." he said. "People are watching their livelihoods wash up on the beach."] IV.24

President Obama still focuses on the economy and livelihoods, but relates it to the actual spill and channels the emotive response that many critics claimed was absent. Unlike the 
voices from BP and the Coast Guard, President Obama's portrayal in media shows his team's attempts to infuse his conversation with talk of fragile coastline and human impacts. President Obama's talk, however, is generally superficial and romanticizes both the locals and the coastline; the narratives do not deal with substantial issues, a timeline for cleanup, or the legal consequences. "We're in this together," he said, gesturing to the three governors, two Louisiana senators, a congressman and other officials he had just met with for more than two hours (Article 22). President Obama suggests there is trustworthy unity in the Gulf response on a visit to the coast, all in an effort to demonstrate leadership.

President Obama eventually condemns BP in anger after several failed attempts to plug the well but does not critique its institutional failings, or even mention the 'culture of risk' (Articles 19-21) that was criticized by the presidential report on the spill. Obama instead focuses on reassuring residents and getting the job done.

\subsection{Conclusion}

This chapter has chronicled the voice and access in a sample of 70 New York Times online articles after the spill. Various media studies, prominently Molotch and Lester (1975), confirm that there is a well-documented trend of over-reliance on expert sources in the news. Chapter Four explored the tendency to rely on experts through a careful examination of sourcing patterns. Sources are a crucial site of sociological investigation into news. This first snapshot and preliminary analysis of data reveals that the top three sources are undoubtedly the federal government, BP and locals. By one measure, locals have the highest representation, but constraints such as the frequency with which they are quoted in the articles and the diversity of the local voice versus BP 
have a major impact on how the data should be interpreted. Likewise, locals are presented as simplistic and emotional rather than concerned with the broader social implications of the BP spill. The main sources are often shown as critical of the spill, passionate, emotional, angry and focused on the short-term events at hand. On the other hand, experts focus on the technical details of the spill. There appears to be little deviation between experts and environmentalists or conservationists, whose voice is infrequent. When considering the process of categorization, this chapter reflects a clear reliance on authoritative and expert sources, which thus legitimizes their authority over that of general residents. The implications of this first stage of analysis will be discussed in greater depth in Chapter Five.

It is imperative to the frame analysis in Chapter Five that I explore the construction of the major sources, and examine the over-reliance on official and authoritative sources. I must likewise give specific attention to the portrayal of nonofficial sources, such as the various voices that make up the local category. The findings reveal that the major sources outlined above do not discuss the causes or repercussions for the spill in the long term, nor do they discuss the political and social implications for the spill. Thus, some alternate framers like locals and environmental groups have access to the news, but the content of their voices as constructed in the sample does not connect the spill to the broader structure that led to the event. Locals are presented as emotional and unengaged with the broader structural issues at hand that pertain to the spill. The federal government assures Americans that the spill will be dealt with and condemns BP. Alternately, BP blames other corporations while minimizing the spill and assuring it will be cleaned up. 
The data presented in this chapter has brought to light methodological questions about organization. presentation of data, and the difficulties inherent in comparing divergent institutions and actors. These problems are highlighted specifically by the comparison of $\mathrm{BP}$ and locals who are such dissimilar groups that it is nearly impossible to conduct a fair assessment of their competing access using quotation patterns alone. In particular, it would have been short sighted and incomplete to only measure the total number of quotes without then considering how many total articles within which each category is quoted as well as who is quoted first. The analysis of voices adds a crucial dimension to the numerical analysis in the first part of chapter. The next chapter presents the key frames and themes present in the articles and links this information to the observations made in this chapter thus far. 


\section{CHAPTER V}

\section{Frames and Themes}

This chapter takes a detailed look at the broad frames and themes that emerged from the data. Unlike Chapter Four, which focused on the portrayal and access of different key sources, this chapter looks at how the content of the articles presents broad framing packages. As was discussed in Chapter One, I began this project with the tentative argument that news coverage ignored the systemic causes and consequences of the BP oil spill. I suspected that the coverage instead prioritized voices that framed the causes for the spill as individual and technical. This tendency remains crucial to the project, but as a component part of a broader argument. As I began to interact with the data, constructed a coding scheme, and then began to cluster those codes, several key frames emerged. After I returned to the literature several times, it became clear that these frames could all be linked to the thinking and priorities of neoliberalism.

My argument subsequently evolved to suggest that the New York Times coverage of the BP spill reproduces various narratives that are all component parts of a broad neoliberal ideology. Although neoliberalism is evidenced in culture at many levels, this premise evolved from a series of key tendencies noted within the literature on neoliberalism. Broadly, these include the tendencies to place every problem and solution in financial terms, value the economy over every other social need, focus on flawed individuals over flawed systems, and promote a deepening of the neoliberal economic status quo. All of these features of neoliberalism are manifest in the data.

The major questions in the aftermath of the spill were: who is responsible, why did the well explode, how bad is it, who is going to fix it, and how? Prominent sources 
proposed a wide range of answers to these questions. Generally. the proposed explanations and solutions demonstrate neoliberal ideology through four key themes. These tendencies are to: a) individualize the coverage and focus on exceptional explanations; b) prioritize business solutions and the economic status quo; c) minimize the spill magnitude and; d) omit alternate frames and structural narratives. These four broad themes overlap and are not all encompassing, but serve as organizational categories, which illustrate the emergent neoliberal ideology. These major categories of frames are not mutually exclusive, nor do they represent all of the frames that emerged from the data. Through these major frame categories, I explore competing media access while critiquing the presentation of the spill by both the New York Times on the whole, and the views put forward by sources.

As was outlined in greater detail in Chapter Two, I employ the term frame flexibly in the analysis. I understand frames to be narratives that are promoted by particular sources. Journalists and sources both participate in framing by promoting specific versions of events. Frames are often connected to a broader ideology. I restrict my definition of the discourse to pieces of talk or utterances, which are then compiled into narratives. Within the scope of this project, it is unnecessary to place strict parameters on the size of a frame; rather, a frame is a narrative (or interpretive package) that is advanced by a particular source, regardless of its size and scope. I am especially interested in these source narratives (or frames) when they can be linked to a broader ideological system. The initial coding process revealed hundreds of narratives and frames, but after returning to the codes, themes emerged, which could likewise be referred to as frame clusters or framing packages. 


\subsection{Individualizes Coverage and a Focuses on the Exceptional}

As outlined in Chapter Two, a key facet of neoliberalism is the tendency to individualize social problems. Stringent supporters of neoliberalism focus blame and responsibility on a few guilty individuals and "bad apple" corporations without exploring systemic blame and responsibility. The featured sources in the sample often blame the spill on flawed people, specific faulty technologies, or suggest the spill was merely an unavoidable accident. Sources in the sample generally condemn BP, but fail to discuss the broader circumstances that shape the organizational corporate practices of companies such as BP. Some sources explain the spill as a product of exceptional and unlikely circumstances. Journalists reproduce these frames due in part to their reliance on the objectivity paradigm. Journalists feature key sources and thus objectively report 'the facts.' As was explored in Chapter Two, this journalistic paradigm enables the author of the article to rely on quotes from key sources, and the author is likewise discouraged from editorializing (Schudson 1989; Carlson 2009). This paradigm decreases the critical discussion in media and reinforces the importance of relying on authoritative and official sources. As was explored in Chapter Four, official sources such as BP and the federal government, promote the above frames.

The articles were often focused on the minute details of events that punctuated the progress of the spill, such as a local seafood market. (Article 12) corporate donations to hair booms, (Article 7) and even Tony Hayward's mannerisms and dress while he addressed reporters on the Gulf (Article 13). These details are doubtless important in some cases, but when they become the focal point of the article, they obscure broader themes. As was explored in Chapter Two, media narrowly focusing on details and the 
experiences of specific people often trivializes the focus of a story (Jewkes 2004). The coverage in this sample often focuses on the culpable individuals and groups rather than broader economic and social problems that play a large role in the causes and responses to the spill. The news coverage focused the readers' attention on these trivial details to the neglect of a more in-depth conversation. Examples of coverage with an individual focus are further illustrated in the subsections to follow. These subsections are useful for organization in order to illustrate specific frames that are advanced by sources and journalists, all located within the broader framing package of individualized coverage.

\section{Human Error}

The tendency to blame large disasters on specific human errors is a prominent BP frame following the spill, but also a well-documented response to disasters. As described in Chapter Two, Anderson (2002) explains that

after the Exxon Valdes spill, media coverage

focused on the drunken captain, who became a

scapegoat for the company. Article 16 explains

how BP and the Coast Guard investigated rig

workers after the blast and forced all workers to

participate in a drug test. The article suggested
Article 40: Andrew Lynch, a fund manager at Schroders in London, said one priority should be to conduct a "thorough review of procedures within $\mathrm{BP}$ to make sure that the message of safety is clear among the people who are out on the drilling rigs.

Box V.1 that: "The search for an explanation would begin with them. That search continues." Even before surviving rig workers were allowed to visit their families, their behaviors were targeted as a potential cause for the accident. The immediate drug test demonstrates BP's interest in discovering a sensational but individual problem on the Deepwater Horizon. 
A quote from within the financial industry (see box V.1) simultaneously reflects the sentiment that $\mathrm{BP}$ can be easily reformed. and likewise blames individual $\mathrm{BP}$ employees by suggesting that if they all receive the message of safety, the company will be accident free. However, even early on in the coverage many of the proposed explanations from experts outside of BP focused on failures of safety systems and technologies, not poor judgment calls by employees. As BP assembled its investigation into the spill, the company continued to claim that there were individual errors at play. In

Article 43: "It's a very complicated industrial accident," Mr. Dudley said during a telephone interview with reporters. It resulted from "a series of individual misjudgments by very experienced people and a multiple series of failures of equipment and processes of using equipment that is going to involve multiple companies here."

Box V.2
Article 45 CEO Tony Hayward says: "It is clear that this accident was the result of multiple equipment failures and human mistakes involving many companies." Hayward bluntly suggested that there were mistakes, failures, and multiple companies to blame for the spill.

New BP CEO Robert Dudley implied that individual errors and misjudgments caused the spill (see box V.2). His quote minimizes the potential effects of systemic problems on the rig. He also says that very experienced employees made the mistakes, and thereby suggests that it was an unlikely and unavoidable accident, rather than the result of any BP negligence. Dudley reinforces the prominent $\mathrm{BP}$ frame that multiple companies were to blame, as is explored in greater detail later in this chapter. Although several articles cite the ways in which Dudley intended to transform the embattled corporate culture left behind by Hayward, 
they likewise demonstrate that the two CEOs have nearly indistinguishable explanations for the spill.

The passage in box V.3 continues with this trend of focusing on specific personnel and even names a guilty party. Despite

the multitude of complicated technical reasons that were discussed in many articles, this passage implies that a single man made decision caused the accident and simplifies the explosion. The article contributes to the BP frame that human error is to blame by implying that errors in judgment can account for the spill, and overlooks the possibility that bad decisions could be the result of external pressures or inadequate training.
Article 64: Together the two men oversaw critical tests in the two days leading up to the explosion, and Mr. Vidrine, who

is 62 , overcame his apparent doubts about the well's integrity and made a momentous decision that led to the accident, according to the testimony of others. He gave the order to replace heavy drilling mud in the riser pipe, which leads from the rig to the well's head, with lighter seawater, a necessary step before capping the well.

Box V.3

\section{Corporate Bad Apples}

One of the most common frames in the sample is that BP is at fault, a view often shared by locals, government officials, and other corporations. Many articles cited sources who blamed BP rather than blaming specific employees or human error. Although this framing process is critical of BP, it still contributes to individualizing tendencies in the spill coverage by failing to discuss the regulatory structure or economic climate that may have contributed to the spill. BP frequently accepts responsibility for the cleanup, but not for the spill itself. Although the general tone in the sample presumes BP is at fault, sources seldom discuss why there was an oil spill in the first place. Many sources express a desire for BP to be held accountable, pay for the cleanup, and 
compensate residents. As was demonstrated in Chapter Four, these same sources often convey hopelessness about the potential long-term effects of the spill. This frame is often implicit because the sources seldom explicitly discuss responsibility or causality.

In late May, the discussion is less optimistic. On May $29^{\text {th }}$ the top kill procedure had officially failed and BP was not ready to begin another option for plugging the well. The articles cited participants who condemned BP for not knowing the mechanisms of their own deepwater well better: "[An anonymous BP technician] added that engineers never had a complete enough understanding of the inner workings of drill pipe casing or blowout preventer mechanisms to make the efforts work" (Article 29). The technician suggests that BP was unprepared for a spill and none of their capping procedures were likely to work. These sources never clearly blamed BP for causing the spill, and instead highlighted their negligence in being unprepared.

Article 20: But BP, the nation's biggest oil and gas producer, has a worse health, environment and safety record than many other major oil companies... Despite those repeated promises to reform, BP continues to lag other oil companies when it comes to safety, according to federal officials and industry analysts

Box V.4
As was previously discussed in Chapter Four, in the relatively infrequent case that locals assigned blame, it was almost exclusively directed to $\mathrm{BP}$ and focused on financial compensation. One man from Louisiana demanded compensation in a town-hall-style meeting with BP and Coast Guard officials. He said: "Are you going to take care of all the oysters I lost?" (Article 30). Although locals

were often cited mourning the loss of their way of life and occasionally demanding help, they seldom assigned blame. 
The federal government frequently blamed BP for the spill. The Coast Guard and BP worked very closely on the cleanup and were often quoted as a single voice, but the government response (particularly President Obama) gradually became more frustrated with BP. While BP often blamed Transocean and Halliburton, these corporations took aim back at BP. Transocean suggests that BP sacrificed safety to save money: "[BP made] cost-saving decisions that increased risk — in some cases, severely" (Article 66). This could be perceived as pointing to a systemic problem, but Transocean only suggests that this is an isolated string of decisions within BP, and despite the apparent lack of safety, continued to lease their rig to BP and allow Transocean employees to work on board.

BP was often framed as a delinquent corporation with a poor safety record and in need of reform. However, these calls for reform generally suggested that BP could be fixed within the current system. Framing BP as deviant is a component of the frame that blames BP for the spill and also contributes to the overall individualizing of social problems. Although BP is framed as having systemic problems, this criticism is not extended beyond the individual company (see box V.4). These quotes clearly demonstrate that BP lags behind other corporations and is consequently a 'bad apple' corporation that should be reformed. Many sources suggest that certain deviant personnel or management are the root of BP's problems. BP contests the allegations that they are a particular example of an unsafe corporation. Article 20 cites their former chairman: "what I saw were breakdowns in a culture of safety, but to say there was something systemic -- I couldn't see that." He uses the phrase "culture of safety" but then explicitly denies that this indicates a systemic problem. When discussing BP's safety problems, one 
industry source praises the superior safety

procedures at Exxon and refers to the company as

the industry standard. Although there are safety

issues at BP across the board, this strongly

reinforces the idea that BP is a 'bad apple'

corporation that must be reformed. This discourse

explains and justifies the explosion, but forgives

Article 40: "If Congress wants to make sure bad actors don't continue to get leases, then it is important it passes legislation to ensure that," said Marilyn Heiman, director of the Offshore

Energy Reform Project at the Pew Environment Group. "And it would help provide an incentive to industry to not cut corners." and celebrates much of the rest of the oil industry.

Box V.5

An environmentalist (see box V.5) reinforces the notion that certain oil companies are problematic and that legislation can help to prevent these operators from causing future problems. She adheres to the business model by suggesting that isolated problematic actors can be reformed through incentive and legislation, rather than

Article 41: It is unclear whether Mr. Dudley, who joined BP in 1998 when it acquired Amoco, can drive the fundamental cultural change that many analysts and even some company insiders say that BP badly needs... Inside BP, Mr. Dudley faces the challenging task of improving the morale of shaken employees while reforming a stubborn culture of excessive risk-taking that placed speed and profits ahead of safety.

Box V.6 exploring the notion that deepwater drilling might

be inherently problematic, as many

environmentalists do in alternative media sources. Article 42 is titled, "Now to Clean up BP", which implies that the Gulf has been dealt with, even though the oil had only stopped flowing days earlier and due to a temporary cap.

Midway through the progression of the spill, BP announced that they changed their CEO. Many sources suggest that with their new $\mathrm{CEO}, \mathrm{BP}$ will

be reformed (see box V.6). The passage suggests that a CEO can drive a cultural change 
within a corporation. The journalist clearly places blame for the aforementioned disasters on BP's culture of risk, which prioritized profit over safety, but then proposes individual solutions to these deep-rooted cultural features of an oil company. Many large oil corporations became enormous by prioritizing profit over safety. All of the big operators in the Gulf had inadequate spill cleanup and contingency plans at the time of the spill (Markey 2010) $)^{2}$ BP has made a habit of repairing public relations smears with a CEO change: Tony Hayward was promoted when their Texas City Refinery exploded. At the time, he also promised a new era of safety for BP (Wardell 2010). BP's actions suggest that an individual can be used as a remedy to overarching problems.

In reaction to the spill, US senators proposed a bill that suggested environmental reforms and stricter penalties for deviant corporations. The coverage in Article 46 explains that the bill addressed problems within

Article 46: It includes a so-called bad actor provision that would deny drilling rights to any company that has had more than 10 deaths offshore or at landbased oil operations over the previous seven years. That rule would currently apply to only BP.

\section{Box V.7}

the drilling industry, including overhauling offshore drilling, lifting liability limits, and increasing funding for research. However, the article then reads: "The narrowly written Senate bill falls far short of the comprehensive legislation on energy and climate change that Senate

Democrats and the White House have been pursuing for more than a year" (Article 46).

The article details a 'bad actor provision' (see box V.7), which would prohibit oil companies from operating after they have had an accident that killed more than ten

\footnotetext{
2 The spill clean-up plans from all major operators in the Gulf were revealed to be nearly identical. They all prioritized Walruses which have not lived in the Gulf in millions of years, and listed emergency contacts that were long deceased (Markey 2010).
} 
people. Although this coverage extends potential culpability beyond $\mathrm{BP}$, it nonetheless

individualizes the coverage by focusing on

Article 67: The most significant failure at Macondo - and the clear root cause of the blowout - was a failure of industry management," the study concluded. "Better management of decision-making processes within $\mathrm{BP}$ and other companies, better communication within and between $\mathrm{BP}$ and its contractors and effective training of key engineering and rig personnel would have prevented the Macondo incident.

Box V.8 particular deviant corporations.

BP predominantly blamed Transocean, and even suggested that the company was solely responsible for the accident: "In interview after interview, Mr. Hayward repeatedly points to Transocean, the owner of the rig that exploded, as the company ultimately responsible for the damages. But at the same time, he guarantees that: "BP will spare no efforts to clean up the mess"

(Article 20). Again, BP suggests that they will voluntarily clean a mess they insist they did not create, which is a virtually nonexistent practice in the corporate world. However, BP was primarily focused on the spill as a public relations disaster. In the September sample, after the oil had stopped flowing, BP continued to deflect blame onto other companies in their internal investigation ${ }^{3}$. The White House commissioned their own investigation from the Presidential Panel, whose report briefly discusses oil industry problems, but still focuses on specific corporations. Although the report discusses

\footnotetext{
${ }^{3}$ Article 66: "BP spent months this summer trying to contain the gusher of oil on the floor of the Gulf of Mexico. Now the company is trying to contain the legal and financial fallout from the explosion of the Deepwater Horizon, and on Wednesday it released the results of an internal investigation that mostly pointed fingers at other companies."
} 
deficiencies in corporate cultures, it is only on the level of specific problematic companies. $^{4}$

The Presidential Panel gives condemning commentary about BP's failed culture of safety, and Transocean's lack of maintenance, but does not subsequently link the discussion to the problems of self-regulating industry

Transocean's equipment documents reveal for the first time the severity of the maintenance issues that plagued the Deepwater Horizon, and they indicate that the company was aware of the consequences of the problems... These new documents refer to at least 36 pieces of equipment in ill repair on the Deepwater Horizon that "may lead to loss of life, serious injury or environmental damage as a result of inadequate use and/or failure of equipment Box V.9 (see box V.8)

Some articles focus on Transocean as the 'bad apple' rather than BP. Internal documents from Transocean's safety record began to surface during the spill investigation. The quotation in box V.9 reveals that Transocean was aware of the Deepwater Horizon's safety issues and that equipment was not being adequately repaired. These were not isolated problems on a specific Transocean rig, but were

revealed to be company-wide problems.

A passage from Article 56 reinforces the notion that a breakdown in the human culture and staff relations, along with poor training can be blamed for problems in Transocean: "A lack of hands-on experience for workers and a stifling bureaucracy imposed by onshore management has led to widespread resentment among rig workers." Even on the small scale, workers suggest in the article that Transocean only investigated problems in order to attribute blame. Although it sounds as if the report could be

\footnotetext{
${ }^{4}$ Article 67: "The report did not pin the accident on any one of these mistakes, but rather attributed it to a broader breakdown of communication and a lack of a culture of safety at the companies involved."
} 
extending its lens to the drilling industry on

the whole, it only suggests that there are

problems across Transocean's rigs. The article

goes on to suggest that problems with

Transocean's maintenance may have

contributed to the sinking of the rig in the first

place, and some sources suggest that the well

may never have leaked had the rig not gone
Mr. Hayward made much the same argument, hitting back at

other oil companies that had distanced themselves from BP by criticizing its choice of a cheaper and riskier well design. "I genuinely believe that there are some issues the industry needs to reflect on, such as the extent it pushed technology and procedures," he said (Article 43). Box V.10

down. Thus, the article focuses prominently on the Deepwater Horizon and its particular deficiencies.

A solitary statement in the sample came very close to blaming the drilling industry for the spill. This criticism came from Tony Hayward rather than a local or environmentalist (box V.10). Hayward may appear to be criticizing the industry, but he

Article 26: Two weeks after the rig sank and oil started gushing into the Gulf, Mr. Perry called the accident "an act of God" and warned the federal government not to be too hasty in halting offshore drilling operations until the cause was known. Box V.11 does not suggest it should change. Hayward is aware that the 'corporate culture' at BP is industry wide and is retaliating against other companies for suggesting that $\mathrm{BP}$ is an isolated problematic company with major safety issues. This quote is one of the most revealing in the sample since

Hayward's experience privileges his insider knowledge that all oil corporations in the Gulf operate under the same mandate. It is likely that his criticism was borne of frustration and only meant to indict the other companies including Transocean and Halliburton, which had blamed BP in the press. 


\section{The Spill as an Unlikely Accident and an Act of God}

Many sources and stories suggested that the spill was an unlikely accident that could not have been prevented, which also contributes to the broader framing package that the spill was an isolated and exceptional event. In the initial days following the spill, several articles and sources suggested that the rig was technologically sophisticated and thus unlikely to explode. The sources often discuss how clean the safety record of the cutting edge Deepwater Horizon had been and how little had gone wrong, although later coverage revealed that the rig had been plagued with problems, as revealed above when condemning Transocean documents emerged. The first article in the sample explains that the Deepwater Horizon was one of the largest and most sophisticated drilling rigs in the world and that no other accident like this had ever happened. Likewise, the Minerals Management Service (MMS) confirmed they had recently conducted a routine inspection and the rig was unproblematic. ${ }^{5}$ A local repeats this claim in Article 35: "The best minds in the world are supposedly working on this," he said, "but no one has seen anything like this before." This local advances the frame that the event was an unlikely accident that could not have been accounted for.

Article 16 focuses on the experience of drill workers the night of the blast, but still finds space to brag about how wonderfully Deepwater Horizon had performed, and implies that it was an unlikely rig to experience an accident. Workers
A 35: Asked how he felt about the oil spill, Mr. Hayward said that the company had worked hard to improve its safety standards in the last three years. But, he added, "sometimes you step off the pavement and get hit by a bus." Box V.12

\footnotetext{
${ }^{5}$ Article 1: [Mr. Hayes said the Minerals Management Service had performed three inspections of the rig this year, including one in the past month, and found no cause for concern.]
} 
were excited that the most recent procedure had gone so well and that they would get to go home early, although it was revealed later in the sample that the drilling had been behind schedule. The Governor of Texas encapsulates the view that the spill had been an unlikely accident (see box V.11). Governor Rick Perry suggests that the spill is completely out of the hands of human control by calling it an act of god. He also suggests that regulation is risky. He frames the spill as blameless, and encourages the reader to accept that accidents happen and cannot be avoided.

Despite mounting evidence to the contrary, expert voices implored the reader to believe that the spill was a freak event, one expert even suggested that the spill was: "beyond the realm of expectation, most likely a combination of unimaginable human and mechanical error" (Article 28). This scientist suggests that there would have been no way for BP to prevent, predict or prepare for the blowout. Likewise, Tony Hayward suggests that the incident was unpredictable and that BP had worked hard for a culture of safety (see box V.12). He crafts the situation to appear especially accidental by reducing it to a simplistic metaphor of being hit by a bus, which suggests that BP is the actor who had been the victim of a tragic, unpredictable accident.

Many sources suggest that deepwater drilling is: "inherently more dangerous than many other industries" (Article 20) and thus accidents are to be expected. A scientist in Article 28 says that the risks will never be zero. The frame that deepwater drilling is inherently risky contradicts the above suggestions that the spill was unlikely, but it depoliticizes the spill and suggests it is a blameless event. Although many authoritative voices suggest that the drilling is dangerous, they seldom propose solutions, and never suggest that drilling be stopped. This theme persists through the duration of the sample. 
The above examples and discussion clearly

describe how the spill was framed as an individual

Article 12: Along the Gulf

Coast, where dinner

and exceptional event by many sources.

Specifically, sources blamed individual workers.

suggested different isolated corporations were

'bad apples', or suggested the spill was a freak

incident without cause. These tendencies remove

the spill narrative from economic, political, and

social history. This is intimately linked to the conversations swing quickly

from anger toward BP to opinions on the best places to eat brown speckled trout, petroleum and seafood have long been intertwined. The two industries rely on each other. Oil money sustains both the roadside seafood shacks that feed a rig worker's family and the restaurants designed for expense-account lunches. Box V.13

tendency to omit structural narratives, as explored in section 5.5.

\subsection{Prioritizing Business and the Free Market}

This framing package is explicitly neoliberal and proposes that the market rather than government protections will guide society through crisis. Sources who promote this frame suggest that corporate practices are not responsible for the spill and insist that a continued focus on business is the only way forward. These sources promote an unregulated free market, in the absence of government oversight. Generally this frame encompasses a deepening of the status quo, and often duplicates the tendency to blame the spill on flawed individuals. However, generally the proposed solutions in a business frame are to decrease or eliminate government regulation, which is a structural, rather than individual response. In other words, it would be wrong to say that there is no systemic critique altogether; instead there is sometimes a systemic critique, but a different kind of critique - one that suggests the situation is not neoliberal enough. 


\section{Prioritizing only the Economy and Business}

The coverage often focuses only on the economic repercussions of the spill. There is frequent discussion of how BP will survive the financial consequences of the spill, what the costs will be to locals, and calculating the environmental costs. A prominent consequence of neoliberal economic ideology is the tendency to understand resources, problems, and ecological commons in market financial terms. As explained in the review of the literature, this is part of a broader tendency that places every element of social life in financial terms. Thus, social and environmental resources can be commodified and privatized. By this same logic, all problems can be solved once profitability is resumed. This section explores how the news sample reproduces this tendency to reduce social and environmental problems to financial losses and gains as well as to prioritize industry, the economy, and jobs above all else.

The text in box V.13 suggests that the oil and seafood industries rely on each other without explaining how or why. The article

Article 13: The stock of the London-based oil company has plunged, and officials in Washington are promising tighter regulations. On Thursday, Interior Secretary Ken Salazar said the government would not issue any new permits for offshore drilling for at least three weeks. and it was unclear how much Congress might limit deepwater oil drilling, a big source of profit for BP and tax revenue for the government, in the future Box V.14 suggest that the local seafood market could not survive without oil rig workers to consume their products, but there are no statistics on what proportion of locals work in the oil industry, and these employees would likewise support all local industry, not seafood in particular. The article does not explain that employees of the oil industry eat any more seafood than other locals, nor does it explain how the oil industry relies on seafood 
beyond sustenance for their employees, who presumably consume a full range of food.

The article provides no evidence that the oil industry would suffer if the fisheries were to disappear. However, the implication is that although locals are angry with BP, they cannot simply condemn oil and gas because the fisheries and oil are in a symbiotic relationship. ${ }^{6}$ This discourse reinforces a pro-business frame.

The quote in box V.14 prioritizes the economy. It also suggests that regulation of the oil industry could harm the tax revenue of the U.S government, even though the only proposed regulation at this early point in the spill was a temporary moratorium on new drilling, not on Article 19: It is too early to tell how the maddeningly unpredictable oil slick in the Gulf of Mexico will affect the economies that depend on the ocean and its edible inhabitants. And it is too early to judge how

$\mathrm{BP}$ will act in providing compensation for economic losses Box V.15 completing wells that already existed. However, there was no discussion of how much income tax BP actually paid to the US government in previous years. No article explained if corporate tax revenue comes close to compensating for the potential long-term costs to the host country of a spill. At the time of this writing there is still a $\$ 75$ million dollar cap on BP's liability. Some senators had suggested increasing the cap to $\$ 10$ billion, but none argued that there should be no cap at all. The presence of any liability cap implies that the government could eventually end up paying, rather than BP having unlimited potential liability to accompany unlimited potential damages. All the while, BP is still afforded the responsibility of internally determining "legitimate claims" from affected locals.

\footnotetext{
${ }^{6}$ The article also goes on to explain that the rigs attract fish, thus fishermen know that creat catchesare discovered in the water near the rigs. However, this simulated reef could be achieved with any structure in the water and does not increase the biodiversity, but concentrates it. There may have been similar gathering areas before the construction of the nearly 3000 rigs in the Gulf.
} 
Financialization and a cost oriented

approach fails to account for less quantifiable

human and environmental impacts. The health of

the ocean is often only referred to concretely in

financial terms. Box V.15 demonstrates a

common tendency to give little concern for

aquatic life other than the revenue it provides to

the fishing industry; there are many inedible
A 33: One analyst calculated that in a worst-case scenario, BP's cleanup liability would be around $\$ 14$ billion, which would account for the entire loss of all fishing and tourism revenues for coastal states closest to the spill, said Kevin Book, a managing director at ClearView Energy Partners. Even then, Mr. Book said, the market overreacted, and $\mathrm{BP}$ can easily handle the cleanup bill Box V.16 inhabitants of the ocean who are linked intimately in the food chain, but who are implied to be irrelevant. The oil and dispersant may present irreversible damage that cannot necessarily be compensated for through a fund. Box V.16 shows a clear example of the tendency to view the spill only in financial terms. An industry funded research corporation suggests that the 'market' overreacted. The

Article 26: "I hope we don't see a knee-jerk reaction across this country that says we're going to shut down drilling in the Gulf of Mexico, because the cost to this country will be staggering," he said... but while he has acknowledged the harm the spill could do to the state's tourism economy, he has frequently accused the news media of exaggerations."

Box V.17 analyst employs an extreme financial lens by considering only the revenue associated with the damage.

There are specific examples of two local politicians who were explicitly pro-business. Texas Governor Rick Perry is described as having long taken a stand against environmental regulations. He called the accident "an act of god" two weeks after

the explosion. Governor Perry suggests the only threat from the oil is an imagined one, promoted through the media hysteria and subsequent loss of tourism revenue or by 
reactionary policy writing (box V.17). He explicitly prioritizes the economy, and uses political scare tactics in an effort to sway the public to sympathize with the importance of the oil industry to their livelihood. He suggest that the costs to the country will only be great if deepwater drilling is stopped or slowed, and presumably believes there is little cost associated with the spill itself by comparison. After the president imposed a sixmonth moratorium on new deepwater wells, Perry demanded that oil drilling in shallow areas be reopened. ${ }^{7}$ Governor Haley Barbour of Mississippi criticized overreaction rather than BP or the oil industry. He was quoted saying: "A bunch of liberal elites were hoping this would be the Three Mile Island of offshore drilling." He accused the news media of exaggerating the disaster, described the oil on the Gulf as "very, very, very thin" while dismissing comparisons to Exxon Valdez (Article 26). By downplaying the effects, mocking environmental regulations, and framing the incident as an accident, these state Governors repeat the frames that the spill is an unlikely accident that could not have been predicted.

One segment late in the sample implies that the Gulf benefited from the economic stimulus of the spill: "Billions of dollars have poured into the Gulf during the response, supporting coastal communities that have had a dreary summer but also enriching contractors involved in the cleanup. Any news of dissipating oil hints at a looming end to that" (Article 60). This author frames the talk as though the slow removal of the oil from will have a negative effect on the Gulf. This passage blatantly ignores the reality that BP was only paying out 'legitimate' claims for lost income, and thus the spill would not have had a positive economic effect on the area; it would be neutral at best. It seems doubtful

\footnotetext{
${ }^{7}$ On closer inspection the six-month moratorium appeared to be no more than a public relations effort on the part of the government since it was so temporary and only affected new wells. (double check this)
} 
that the stimulus provided by cleanup work compares to revenue lost in tourism and lost jobs. The tendency to frame the spill as an economic stimulus blatantly disregards the human and environmental harms, and thus explicitly prioritizes the market. Even Tony Hayward was seen by those in the oil and financial industry as having a positive effect on the company: "Still, Mr. Hayward was well liked by many investors until the explosion in the Gulf. He cut costs at the company and significantly improved its profitability" (Article 40). Investors fail to link Hayward's cost cutting with the causes of the Gulf spill in the first place. This quote reflects an orientation toward profit that is so pronounced it fails to consider the broader context of the spill.

\section{Decrease Government Regulation on Industry}

Another symptom of neoliberal ideology in culture is a push to decrease government regulation of industry. Proponents of neoliberalism support the notion that the oil industry should self regulate. An ideal free market would see companies governing their own corporate conscience and trusted to validate their own safety and environmental regulations. The rationale is that corporations are motivated internally to promote their own safety because accidents are costly and damage public relations. Companies suggest that they are guided by corporate social responsibility and should be trusted to selfgovern in the absence of regulation. BP frequently frames the disaster as one of public relations, and suggests that the harm can be undone through corporate social responsibility and the good faith that BP will clean up the mess. BP and many other large oil firms do not even have insurance because of how enormous the companies are, the level of risk involved in the work, and how expensive it is to cover in the event of an accident. 
BP communicates their concern about protecting their image as corporate citizen, while the existence of corporate 'citizenship' is a neoliberal phenomenon that responsibilizes each individual person or company for the consequences of their social participation. Mr. Hayward is quoted in Article 5 saying he wants to "win the hearts and minds" of the people. One article suggested that while BP could survive the financial fallout, they would be held guilty in the court of public opinion for years. ${ }^{8}$ This reflects the sentiment that public relations are important to the company beyond their potential impacts on lost revenue. The notion of corporate social responsibility has been intensifying in tandem with the neoliberal push to reduce government oversight: indeed neoliberal Article 15: Last year, BP, the owner of the well that blew up in the Gulf, teamed with other offshore operators to oppose a proposed rule that would have required stricter safety and environmental standards and more frequent inspections. BP said that "extensive, prescriptive" regulations were not needed for offshore drilling, and urged the minerals service to allow operators to define the steps they would take to ensure safety largely on their own. Box V.36 "corporate social responsibility" can mean leaving large corporations to simply govern their own activities.

The quote in box V.36 reveals that BP and other corporations worked together to oppose stricter regulation. Although the journalist begins to discuss the possibility that lax oversight is not restricted to BP, the article does not discuss the implications of corporate control in government. The conversation quickly shifts back to BP and their assertion that extensive regulation was unnecessary. It is no secret that the oil industry spends huge sums of money lobbying the federal government. Self-regulation is a direct

\footnotetext{
${ }^{8}$ Public relations are more important to BP than to companies like Transocean and Halliburton because BP is the only one that sells product to the public; they operate a chain of gas stations across the United States.
} 
and clear consequence of neoliberal economic policy that would have a smaller federal government, reduced oversight and a free market. The coverage documented BP's willingness to change on their own, rather than through prescriptive regulation. The $\$ 20$ billion dollar compensation fund is framed as charitable by many sources in the coverage, but it preempted government and regulatory demands for compensation and would not necessarily occur again were there to be another similar spill.

The Minerals Management Service (MMS) trusted BP to self regulate. Article 15 implicitly blames the MMS for the disaster and points out that that the regulations of the federal regulatory body were barely enforced. However, there is no discussion about why the regulatory environment is lax nor do any sources suggest that this may be a part of a systemic pattern in the relationship between government oversight and corporations. The brief discussion featured in the sample may allow readers to believe this is a particular short-term problem between the current staff of the MMS and BP. Article 15 explains that the MMS continued to allow the industry to police itself because the oil industry employs the best people and has the best technology. This discourse implies that the capacity to regulate is actually out of the hands of the government.

The tendency to trust business to self regulate is reflected in the spill response itself: "The administration has left to BP most decisions about how to move forward with efforts to contain the leak" (Article 30). Despite the federal government's insistence that they are in charge of the cleanup, they admit to defaulting to BP for decision-making. The government concedes that they must default to the oil industry for the technology: "For Mr. Obama, part of the problem has been that the solution to the BP disaster is at its heart an engineering problem, and one the government has already acknowledged it is in 
no position to fix on its own" (Article 36). This passage explains that not only does the government prefer to leave the decision making to BP, but that the government does not have the capacity or technology to oversee the problem. As well, the article suggests that at its core, the spill is an engineering problem. This implies that the problem is shortterm, technical, and disconnected from broader systemic issues.

While there were some state governors such as Hailey Barbour, who openly opposed any form of regulation, some official hesitation was more nuanced: "Several measures have been approved at the committee level, including provisions that would bar operators with serious safety problems from obtaining new offshore leases, a measure seen as singling out BP" (Article 42). The journalist is likely echoing other official voices and suggests that it is unfair to single out BP, even though it is the only operator in the Gulf to have a major accident recently. The proposed legislation discussed above only impacts oil operators who have already had an accident, leaving the regulatory climate for the vast majority of companies unchanged.

Some voices have suggested that the spill will act as a deterrent and as an incentive for the industry to ramp up their safety procedures: "the accident last month would encourage designers and engineers to improve the technology and procedures, so that a disaster like the Deepwater Horizon explosion could not happen again" (Article 28). Part of the rationale for allowing corporate actors to self regulate is the notion that the spill will provide necessary incentive to change their practices.

On the whole, the industry has been afforded the luxury of self-regulating, and that they have received a great deal of cooperation from the government. Article 43 explains that BPs financial losses will be lessened by a $\$ 10$ billion dollar tax credit that 
they received for their cleanup costs, which is not framed as problematic. This sum is half of their total pledged compensation fund.

Along with explicitly prioritizing business and profitability above other concerns, a neoliberal narrative suggests that regulations, especially environmental regulations, will destroy industry. This narrative suggests that jobs and business are directly and perfectly correlated. As previously quoted Governor Perry specifically says that liberal elites wanted to the spill to be the "Three Mile Island of offshore drilling" (Article 26). Perry is suggesting that some liberals want the oil disaster to become a keystone moment that will sully the public perception of offshore drilling and thus to have serious repercussions for future business.

The Democrats proposed a bill for drilling reform. In Article 46 the president of the American Petroleum Institute criticized the Democratic bill, saying that it would raise the cost of production, hurt small and mid-size businesses and enact punitive tax increases. He says: "I would encourage the House and Senate to protect American jobs as they consider appropriate responses to the Gulf of Mexico oil spill." He reinforces the notion that and environmental regulation more generally are hindrances to business and that Americans should prioritize the economy instead. He suggests that jobs will be risked by proposing even modest reform. The appeal to small business owners is often used when promoting pro-corporate policies. Concentration of ownership is pronounced in oil, and 'small' in deepwater oil drilling is relative when compared to the industry standard in the Gulf.

The articles often cite increasing regulation as a threat to Gulf States: "While the states formed an alliance in 2004 to address the Gulf's overall health, the group includes some 
of the poorest states in the country, and they are concerned that tighter rules could chase away jobs" (Article 48). This quote explains that there are people who are interested in the environmental health of the Gulf, but that they

Article 70: The oil industry reacted warily to the report, saying that companies with good safety records should not be subjected to costly new rules and warning that a major new set of regulations would slow production and drive up prices. Drillers also objected to a recommendation that the current $\$ 75$ million cap on liability for accidents be raised by an unspecified amount. V.18 temper that interest with local concern for jobs, which often trumps environment in areas with high unemployment and low household income. This naturalizes the idea that Gulf residents are unconcerned with safety and environment, but the cited pressure is likely political, from voices such as Hailey Barbour and Rick Perry. The passage in box V.18 suggests that tougher regulation will

trickle down into the wallets of the public and affect already high gas and commodity prices. The article does not go on to suggest that accidents would likewise add to prices at the pump, especially when the industry rejects the political will to increase their liability cap. Increased prices would then be passed onto the government and presumably the public.

\section{Business Solutions to Environmental Disaster}

Many articles praise the virtues of technology and reassure the reader that the oil industry is the key to sophisticated and safe drilling. Underlying these frames is the ideological belief that business leads to technology and innovation. Although business may create temporary disruptions in environment and quality of life, these sources frame it as a crucial component of a forward-moving society. This ideology can powerfully 
orient the public interest toward the economy and business, even in the wake of an industrial environmental disaster.

Many sources suggest that a single

accident is no reason to interrupt deep water

drilling or punish other oil companies. These

sources suggest that Americans should maintain

their faith that eventually technology will solve
Article 28: Many experts in the field of undersea oil exploration believe that technology can also resolve the risks of operating tens of thousands of feet under the seabed, despite BP's current problems. Box V.19 their problems and that drilling can be safe. The passage in box V.19 reinforces the notion that technology will outpace risks, and that this problem is isolated because overall, the drilling industry is advanced and sophisticated. The passage also generalizes the expert voice as one that supports technological advancement. The quotes in boxes V.18 and V.19 both present the spill as a minor setback in the impressive progress of deepwater drilling

President Obama is slightly less optimistic than the aforementioned experts, but nonetheless takes for granted that Americans need this domestic source of oil. Rather than problematizing a potentially dangerous industry, he stresses that it must be made safe: "The president was adamant that the government and the industry had to find a way to make offshore drilling safe because the nation needs the oil" (Article 39). President Obama still reflects the attitude that the route to safety can be carved through industry. Despite the massive failure of BPs technology, a BP fund manager suggested that BP belongs in deep water: "Once all that is done, I don't see why BP can't be growing again...Deep water is definitely the right place for them to be. The future lies in the more complex and technically difficult challenges offshore" (Article 40). He suggests that BP 
is best suited the complexities and challenges of deep water drilling once its problematic personnel are infused with a message of safety. This quote reflects faith in technology and science and naturalizes the notion that deep

Article 28: Americans have long had an unswerving belief that technology will save us - it is the cavalry coming over the hill, just as we are about to lose the battle. And yet, as Americans watched scientists struggle to plug the undersea well over the past month, it became apparent that our great belief in technology was perhaps misplaced. ...Americans have a lot of faith that over the long run technology will solve everything, a sense that somehow we're going to find a way to fix it. Box V.20 water drilling is a necessary practice. This financial industry insider suggests that the continuation of the industry should not be subject to interference on behalf of this particular isolated disaster. No sources suggest that BP should be prohibited from continuing to drill for oil and most agree that if the company possesses the sophisticated technology it should continue to use it. These frames are component parts of a broader framing package, which suggest BP is a problematic institution

capable of being reformed. The insistence that drilling not be interrupted explicitly prioritizes business and frames the spill as an isolated technical problem. It is also intimately linked to the section that follows on the American faith that science will fix the industry.

As previously discussed, sources within the sample often present the spill as a technical issue and depoliticize expert voices. This tendency obscures potential systemic problems, and reflects the aforementioned determination to continue offshore drilling. The goes hand-in-hand with the faith that oil industry technologies will fix the problems that led to the spill. In other words, a push to believe in the goals of science and technology is driven by the market, and is thus a component of neoliberal ideology. This 
is another instance in which the facets of neoliberalism directly oppose environmentalism by driving constant progress, technology and economic growth. One article even spoke to the significance that faith in science has in the American psyche (see box V.20).

This passage suggests that Americans still have faith that technological progress and science will save humanity. This lingering faith in the project of modernity (Weber 1992) is connected to the persistent cultural myth of the American dream; that every American can eventually succeed through hard work. The deep-rooted cultural goal of hard work goes hand-in-hand with the aims of neoliberalism, specifically the drive to leave no value or component of social life outside the market. The discussion implies that Americans in particular hold on to post enlightenment optimism that scientific progress will lead to an inherently better society.

This article evokes a war metaphor of American glory and honour, and implies that the oil leak is the villain in an ongoing battle, from which Americans will emerge victorious due to their technological sophistication. The journalist also suggests that because scientists failed to plug the oil leak, this century long faith in technology has been shaken for the first time. Although the failure to plug the oil well may add Article 28: By all accounts, the oil industry is infused with this "can do" attitude: Oil running low? "Oil wells will run dry, but advances in technologies can put off the inevitable," said a 2006 article in a newsletter of the American Oil and Gas Historical Society... Many experts in the field of undersea oil exploration believe that technology can also resolve the risks of operating tens of thousands of feet under the seabed, despite BP's current problems Box V.21 to an overall narrative of technological failure to manage the environment, the mythology has arguably been crumbling for generations with the failure of science to cure AIDS or cancer, to eradicate poverty, or even to free us 
from fossil fuels as an energy source. Despite the sophistication of American technologies, their forces did not emerge victorious in Vietnam, the Gulf war. Afghanistan or Iraq. The battle metaphor which is evoked in the spill coverage is uncomplicated by these politically ambiguous conflicts, and channels successful cultural narratives of WWII or the American civil war.

The above discussion is an ideal metaphor for the overall failure to connect the events of the spill to broader systems. The journalist discusses the idea that American faith in technology is challenged by the failure of scientists and engineers to plug the leak, but does not connect this anxiety to broader, long-term technological failures. Likewise, coverage of the spill is intense and criticizes BP's failure to plug the leak or adequately reassure Americans, but fails to understand BP's inadequacies as consequence of the American economic and political status quo. However, this spill may well prove to be unique. It is the first environmental catastrophe on American soil in the 24-hour, video feed, social media news cycle. Although there have been multiple events in the long march of the industry and technology that may logically shake faith in science, the article suggests American faith in science may now be shaken but also fails to explore why the disaster is a unique blemish on the American psyche. Nor does the journalist consider the repercussions of a newfound cultural distrust of science and technology. The article does not explore the potential drawbacks of perpetual scientific growth or explain that the capacity to drill in deep water is a direct consequence of this innovation.

No article explicitly problematizes science and technology. The articles generally reflect a unanimous faith that scientific progress is good, with differing views of how it should be used. A group affiliated with the petroleum industry suggests that the 
technology can delay the inevitable, and does not

Article 28: Still, as he watched a live feed of drilling mud being pumped into the leaking well on the seabed, he acknowledged that the science of repair and cleanup seemed lacking. "My impression is that we were unprepared for this," he said. "There were not a lot of good technologies and techniques ready."

Box V.22 describe oil accurately as a finite resource (see box V.21). In spite of the blowout, the industry

maintains its optimism that the practice of deepwater drilling can be reformed and should even be expanded. They suggest that technology can manage risks, even though the rapid growth of technologies enabled the industry to drill in extreme

conditions and depths.

One explicitly pro-business voice admits that the technology of repair and cleanup are lagging (see box V.22). The same article suggests that the spill will provide the needed incentive to create these new technologies and is thus an unproblematic glitch in the necessary progress of deepwater drilling.

According to this free market logic it would take

a major spill to develop sophisticated cleanup

technologies. Generally the articles reflect this

same sentiment that now that there has been an

accident cleanup technology will accelerate.

Despite the reality that rapidly advancing

technologies led to the capacity to drill at such

extreme depths, no voice reflects any hesitation

to further trust industry-inspired technological advancement.
It is not clear how much money is available to pay for mental health treatment for parents and children. Kenneth Feinberg, who is administering the BP claims process, has said mental health claims will not be covered. BP is considering requests from Mississippi and Louisiana for $\$ 39$ million to cover mental health treatment through October 2011. Ms. Drury said that her church was starting a family counseling program, but that she had not had any other offers of help (article 51).

Box V.23 
Under neoliberal policy, charities and special interests are increasingly expected to take the place of government oversight (Harvey 2007). This stems from notion that markets and people alike should experience increasing freedoms. Proponents of neoliberalism argue that taxpayers should not have to pay into the state, but rather choose which causes they decide are worthy. This has the dual effect of shrinking government and responsibilizing individuals. Article 49 reinforces the notion that charities should donate to the Gulf, and it contrasts the amount of charitable donation with Katrina or Haitian earthquake. The article suggests that people and corporations should donate to the Gulf spill recovery as they did to Katrina, ignoring the reality that in the case of the spill there are clear responsible corporate parties. This discourse naturalizes the spill by suggesting it is comparable to a biological event such as a hurricane, although much of the damage and lives lost in Katrina could have been prevented were there political will to protect New Orleans from a storm.

BP admits that they will not pay for mental health problems stemming from the stresses of the oil spill (see box V.23). The passage demonstrates that responsibility instead falls on communities and local governments. Not only is mental stress even further removed from finances and difficult to quantify than physical or job loss, but this reflects the neoliberal attitude that people and communities should help themselves, rather than be supported by large-scale government programs. Charities can fill these gaps, and communities are left to deal with the problems facing their residents. The inclusion of discourse demonstrates the moral component of neoliberal ideology. As discussed in the review of the literature, it is mistake to reduce neoliberalism to only an 
economic policy, when it asks a society to fetishize the economy and prioritize it above all other societal concerns as well as to minimize the role of the state in social life.

\section{$\underline{5.3 \text { Minimizes Magnitude }}$}

The way various sources describe the spill lends important insight into their perception of its severity and arguably shapes the public perception of the spill. There is a clear spectrum within discussion of magnitude ranging from those who suggest the sheen is very thin, such as Hailey Barbour or Rick Perry, to those who say the spill is vast and will impact the oceans for decades (Whitty 2010). Thus, the magnitude of the spill is framed differently by different sources and is a contested site for framing. In their study on the Santa Barbara oil spill, Molotch and Lester (1975) argue similarly that minimizing the effects of an industrial accent in media is advantageous to the continuation of the status quo. Specifically, they suggest that this pattern of minimizing makes it less likely for the public to question the legitimacy of the current American practices for resource extraction.

Overwhelmingly, the presentation of the spill magnitude in the sample suggests that the spill was not as bad as the hype suggests, and that the oil is disappearing at a rapid rate. The extreme pro-business neoliberal view of Rick Perry and Hailey Barbour receives limited attention in the sample, but the critical suggestion that the magnitude is so vast it will have long-term, systemic and structural consequences is virtually absent.

Although the oil slick is often described in dire terms in the sample, this presentation is infrequently accompanied by an analysis of its social causes. As discussed above, some articles suggest that the spill is a force of nature like a hurricane. In Article 19 the spill is referred to as "maddeningly unpredictable." There are multiple 
comparisons drawn between the spill and hurricane Katrina, which implies that the spill is likewise a force of nature: "The oil slick, like

Hurricane Katrina before it, is a big, impending

disaster swirling in from the Gulf of Mexico, requiring a response effort involving all levels of government" (Article 26). This passage asks for a 'big government' response, but on the grounds that the spill is swirling in from the Gulf of its own volition. When discussing the debris from the spill, the articles explain that oil debris is the
Article 44: Scientists said the rapid dissipation of the surface oil was probably due to a combination of factors. The Gulf has an immense natural capacity to break down oil, which leaks into it at a steady rate from thousands of natural seeps. Though none of the seeps is anywhere near the size of the Deepwater Horizon leak, they do mean that the Gulf is swarming with bacteria that can eat oil. Box V.24

norm on many coastal beaches, which are

surrounded by giant oilrigs. The coverage does mention oiled animals, but also states that there were many fewer animal fatalities than one would expect given the deaths in other, smaller oil disasters where millions of animals died. These features have the combined effect of downplaying the spill. In contrast, a great deal of alternative media suggested that BP was concealing the extent of animal death and that many animals were simply never collected since the spill occurred in the deep sea (Whitty 2010).

Although previously discussed, there were also responses from Gulf state governors who explicitly denied the spill was severe. Governor of Mississippi, Haley Barbour clearly demonstrates an effort to downplay the spill "We haven't had a milk jug full of oil that's got on our coast..." (Article 26). He suggested that comparisons to Exxon Valdez were hyperbolic, as the layer of oil in the Gulf was very thin (Article 26). 
Very shortly after these public statements the spill was determined to have surpassed Exxon by a large margin.

Once the spill had been capped, the magnitude discussion shifted from a general tone that the Gulf was heavily oiled, to one that suggested the oil was disappearing faster than anyone imagined. These optimistic quotes from authoritative sources appeared in abundance as soon as the oil stopped flowing. BP, government and experts from the scientific community were prominently featured in the coverage supporting the frame that the Gulf is a self-cleaning system and the oil was vanishing. A journalist reinforces this frame by taking for granted that there is a unanimous consensus that the oil is disappearing (see box V.24). This segment naturalizes oil in the Gulf environment, and suggests the hype around the spill was exaggerated. Several experts explain that microscopic organisms consumed the oil at a surprising rate, including dispersed oil, but they conspicuously fail to discuss the effects of the dispersant itself. The scientist says that this positive resolution "has been the story with

The winds from two storms that blew through the Gulf in recent weeks, including a storm over the weekend that disintegrated before making landfall, also appear to have contributed to a rapid dispersion of the oil. Then there was the response mounted by $\mathrm{BP}$ and the government, the largest in history, involving more than 4,000 boats attacking the oil with skimming equipment, controlled surface burns and other tactics.

Box V.25 spills that stay offshore" (Article 55) although no

coverage earlier in the sample had suggested that

offshore spills are harmless. Also, the BP spill

affected miles of coastline and marsh. Many voices

reinforce the frame that the Gulf is a miraculously

self cleaning system and that the oil has almost

completely disappeared (see box V.25).

The coverage also praises the intense BP and government response. This explanation omits the 
role that heavy use of dispersant played in obscuring the oil rather than removing it. The quote uses a battle metaphor and suggests that the oil is the enemy. There is a congratulatory tone toward $\mathrm{BP}$ and the government, who have presumably succeeded in their cooperative attack against the oil. The journalist goes on to explain that although much of the oil has dissipated, there are still risks of a submersed tar ball that poses problems to wildlife. Despite the concerns of locals, the head of the National Oceanic and Atmospheric Administration suggests: "There's absolutely no evidence that there's any significant concentration of oil that's out there that we haven't accounted for" (Article 57). This discussion sheds light on an effort by interested parties to minimize the effects of the spill. The smaller the impact, the less financial and legal fallout for BP. Likewise, public outcry against BP and the federal government will presumably be less severe if the spill self-cleans and disappears from public memory. For both parties this was a battle of public opinion.

It is worth noting that the coverage also features some more dire descriptions of the spill. The spill was being described as the worst in U.S. history (Article 29) and "one of the greatest environmental calamities of history" (Article 33) While there were many articles that described the oil as quickly vanishing (see above), there were also some that detailed the skepticism of the Gulf residents. A local fisherman who had been making money selling anti-BP shirts suggested: "It's not gone... Mother Nature didn't suck it up and spit it out" (Article 60). Some locals had a hard time believing that the oil could simply vanish so quickly. However, these voices were much less common and portrayed as anecdotal and speculative rather than scientific and trustworthy. 
Despite the government assurances that the oil was nearly gone in August, stories from January reveal that the cleanup was ongoing; oil and tar continued to come ashore (Article 69). These voices are a tiny minority of spill coverage, which turned very quickly to the wonders of nature and its cleaning capacity. As well, those who believe the spill is devastating, still seldom discuss potential long-term impacts, and expert voices suggest that it is too early to speculate. Presumably by the time that speculation had been earned, the spill had all but faded from the news.

There were many voices ready to suggest that the media reacted hysterically and exaggerated the spill, which has the latent effect of downplaying the magnitude of the spill. In Article 26 Hailey Barbour suggests that the threat posed by overreaction is more perilous than the spill or botched efforts to cap and clean. One article suggests that environmental groups, lawyers, and politicians are fooling Gulf residents by falsely

Article 60: The skepticism has been stoked by environmental groups that came to the Gulf in droves, lawyers who have been soliciting clients from billboards along roads leading south, a sensation-hungry news media and politicians who have gained broad popularity for thundering in opposition to response officials.

Box V.26 portraying the oil spill as dire (see box V.26). This passage implies that locals have been duped by the hysteria, and that media has overreacted to the spill. Members of the media even began to doubt themselves. As demonstrated above, the Gulf was successfully framed as a self-cleaning system by the Federal Government, BP and scientists.

Subsequently, Anderson Cooper asked himself:

"Has the BP oil spill been overblown by politicians and scientists and media, including me?" (Article 63). This article debates the extent to which the media had overreacted to the spill before anyone started producing research about the long term consequences of 
the spill. One researcher suggested that the BP spill received a disproportionate amount of coverage when compare to the Exxon Valdez (Article 63). Although the Gulf spill had been much greater in magnitude than Exxon, affected much more of the coastal United States and the lives of millions more residents, this researcher argued that the coverage was excessive. He disregards the content of the story and only numerically compares the total stories that featured either oil disaster as a subject. Thus, he proposes that the media has given the spill disproportionate coverage, but this figure includes all stories on the spill, even those that present a pro-business agenda and downplay its effects.

The portrayal of the media as overreacting inherently minimizes the magnitude of the spill. This feeds into a common perception of the American news media that newscasters have an inherent left-leaning bias and would naturally try to exaggerate a spill and BP's consequences. The tendency to minimize magnitude and silence any media that wants to explore environmental issues is a clear consequence of a pro-corporate ideology that encourages deregulation of the industry.

\subsection{Marginalized and Omitted Frames}

Some of the most relevant information in my coding process became the omitted narratives: what was the conversation not being had and where were the links to bigger questions? In this section I discuss the minimal extent to which the media coverage engages structural explanations and solutions to the oil spill. This section encompasses the infrequent coverage that blames and responsibilizes the U.S. government, regulatory bodies, the drilling industry and the broader economy. This section is intimately linked to first section in the chapter on the tendency to individualize the coverage. However, 
efforts to obscure a progressive economic solution extend beyond the tendency to individualize.

The advancement of neoliberalism can also be considered structural change. I will not be exploring that tendency in this section, as I am focused instead on alternatives to neoliberal economics. It is worth briefly problematizing the term systemic. A systemic or structural narrative can emerge from either side of the political spectrum. particularly when discussing solutions to the spill. The solutions could theoretically be to ban offshore drilling and reorganize the economy so it is controlled democratically or to severely limit the powers of the state eliminating any government oversight. These arguments can extend almost limitlessly in either direction, and are all broad systemic solutions. However, as outlined in the literature, this thesis began with the contention that systemic issues were being overlooked and the coverage was focused on individuals. This subsequently evolved to suggest the sample reproduces neoliberal ideology. I suggest that the increasingly neoliberal organization of the global economy created the conditions in which the spill occurred. The argument that future spills would be prevented with less government oversight is an ideological tactic of subscribers to neoliberal economics in order to deepen their preferred economic organization, and it is not omitted.

\section{Coverage that Overlooks Causes and Consequences}

The coverage in the sample often focused on how to recover from the spill and the mechanisms by which residents would be compensated. Although this is necessary discussion, the coverage often focused explicitly on solutions to the neglect of causes and consequences. Exploring the technical, social and economic causes for the spill naturally orients the discussion toward the responsible and guilty parties. Coverage that looks at 
the multitude of reasons the spill occurred would lead to solutions that include mechanisms by which to avoid future spills and address the full range of problems that led to the event.

Rather than integrating blame, many sources proactively look to solutions. However, most of these solutions are inherently short sighted since they fail to consider the reasons the spill occurred. Upon coding the first ten articles, I found that in the beginning of the sample journalists included sources who refrained from discussing the responsibility for the spill beyond pointing out that BP had accepted the financial burden. The articles communicated that BP gives the somewhat paradoxical message that: "It wasn't our accident, but we are absolutely responsible for the oil, for cleaning it up" (Article 5). This communicates that BP was not to blame for the blowout and disaster, but is responsible for dealing with the consequences of the oil. This contributes to BPs framing itself as heroic, as discussed in the previous chapter. Likewise, it removes the accident from discussions of blame and responsibility and suggests instead that the causes are unimportant as a site of focus.

Governor Charlie Crist of Florida suggests that the federal government should ban offshore drilling and look to renewable energy: "For weeks, he has been saying he wants to bring lawmakers back to Tallahassee for a special session focused on a proposed constitutional ban on offshore drilling, and on tax incentives that would encourage renewable energy sources" (Article 26). He implicitly acknowledges that Americans use too much oil, which is the only time this viewpoint occurs in the sample. Crist does not go on to suggest that oil extraction is inherently problematic, but that his state is particularly vulnerable to the damaging effects of offshore oil spills. Florida's economy is 
driven by tourism, and does not have an oil based economy to protect; even the fisheries in Florida, which are also comparatively small. are more important to the local economy than any form of oil production. Due to this political economic context, it is unsurprising that the first voice to reconsider dependence on oil is Florida. He suggests that through technology, Americans need to find a solution to carbon-heavy appetites but does not propose Americans adjust their lifestyles. The Governor focuses on solutions and does not frame overreliance on oil as cause for the spill. Although there may be latent environmental benefits to Governor Crist's proposal, his motivation is clearly an economically motivated effort to protect the tourism economy in Florida.

As discussed throughout the analysis, the democrats proposed a bill in reaction to the spill. The proposed legislation touched on systemic problems in the drilling industry, but it was still predominantly a reaction to the spill and focused more on BP as an individual company than on substantial industry reform. However, the discussion is still some of the most systemic in the sample. President Obama discusses the legislation: "But I want to emphasize it's only the first step. And I intend to keep pushing for broader reform, including climate legislation, because if we've learned anything from the tragedy in the Gulf, it's that our current energy policy is unsustainable (Article 46). Although this statement is vague and may be little more than political rhetoric, President Obama suggests that the American energy policy is in need of reform. He does not go so far as to suggest that the American appetite for oil is unsustainable, nor does he directly condemn or blame that energy policy for failure to regulate in the Gulf. These examples are focused on solutions and do not connect these solutions to the climate that allowed the spill to occur. Although it may be forward thinking to focus on solutions rather than on 
why the spill occurred, this approach is seldom taken with individuals who are framed as deviant or criminal in media, thus it appears to be an effort to overlook the roles of guilty institutions in the spill.

The systemic discussion of consequences often goes no deeper than the possibility that laws were broken in the spill. However, the proposed responses are only to reprimand and fine the law-breaker, be it $\mathrm{BP}$ or Transocean. Legal discussion in the coverage does not pose more complicated questions about why delinquent, criminal corporations are allowed to continue to operate. While some alternative media outlets discussed the possibility of charging BP with manslaughter, the administration only looked into potential violations of environmental laws, including the Clean Water Act,

Article 15: Yet the Minerals Management Service, the Interior Department agency charged both with regulating the oil industry and collecting royalties from it, never took steps to address the issue comprehensively, relying instead on industry assurances that it was on top of the problem, a review of documents shows. Agency records show that from 2001 to 2007 , there were 1,443 serious drilling accidents in offshore operations, leading to 41 deaths, 302 injuries and 356 oil spills. Yet the federal agency continues to allow the industry largely to police itself, saying that the best technical experts work for industry, not for the government Box V.27 the Oil Pollution Act, the Migratory Bird

Treaty Act and the Endangered Species Act

(Article 36). Although it is doubtless

important to consider the environmental

consequences, the coverage only looks at

wildlife mortality rates and pollution. The

government response in the sample avoids

discussing the eleven rig workers who were

killed in the blast and does not discuss the

potential long-term human health

consequences of the spill. This two

dimensional discussion does not encourage readers to consider the broader implications of criminal negligence. 


\section{The Role of Government Regulation}

The tendency to blame government for the spill is often omitted and is inherently more structural than articles and sources that attribute the spill to a single corporation or an unforseeable accident. President Obama frequently suggests that he will be holding parties accountable and that he is ultimately responsible for the cleanup. Likewise, the Coast Guard is never suggested to be complicit in any causes of the spill but has oversight responsibility for the cleanup. The government is often blamed for mishandling the consequences of the spill: "[T]he government and the president appear increasingly impotent as oil laps at the shorelines of Louisiana, and now Alabama and Mississippi" (Article 36). This passage reflects a common sentiment that although the blame for the explosion cannot be determined or is irrelevant, the government is responsible for managing the cleanup and is being blamed for the oil making it to the coast. The opinion advanced in the articles is often that the government should hold BP accountable and that it will ultimately be a government failure if the spill is mishandled. Of those sources that look to government, this is the most common frame.

Occasionally, sources look to the government regulatory body responsible for oversight, primarily the Minerals Management Service (MMS). Article 15 discusses regulatory failure at the MMS, and that they had suggested oil companies implement another failsafe, but they had not followed up and the technology had never been installed (see box V.27). This passage acknowledges that there are problems with allowing the industry to self-regulate and that the MMS weakly enforced toothless regulation that trusted the industry to self-govern. The passage suggest that lack of 
oversight has led to an unsafe environment and the article implies that the industry should not be allowed to regulate itself. but never explicitly makes this point.

The other more explicit calls for government responsibility come through voices that ask where the government regulation was. This local voice comes closest to critiquing systemic failure: "BP is a corporation, it's going to protect its bottom line... but where are the government agencies who are supposed to protect the health and safety of our citizens?" (Article 30). Her criticism looks more broadly than to BP, but Ms.

Article 38: Yet even as such discoveries have multiplied. little has been done to protect the sea life. An environmental impact statement prepared by the Minerals Management Service in 2007 that covered a vast area of the Gulf being opened up to oil and gas drilling, including the lease area where the BP well is located, concluded that drilling posed no serious risk to deepwater reefs. Deep-sea rigs were required to avoid damaging coral sites directly with anchors or pipelines, but few other restrictions on drilling were deemed necessary. The nearly 1,000-page document mentions only in passing the potential of oil released under high pressure to form undersea plumes, despite previous studies showing the distinct likelihood of such an event. The study also failed to explore the application of dispersants deep underwater. This use of the chemicals, approved by federal authorities, is essentially unprecedented. It appears to have reduced the extent of the slick, limiting its impact on wetlands, beaches and surface life. But officials know little about its potential impact on life underwater.

Box V.28

Kuhns forgives BP for their reckless actions and implicitly condones the capitalist model in the United States, which legally requires corporations to prioritize their profit margins above all else. She looks only to the role of government rather than suggesting BP, the MMS and the priorities of the federal government all contributed to a dangerous environment on the water.

In one article the journalist suggests very clearly that the MMS failed to regulate properly, and ignored clear scientific evidence on how to protect the Gulf (see box V.28). 
The journalist included this large segment which suggests that the Minerals Management Service had ignored scientific research that demonstrated an underwater plume would be likely in the event of a deep water spill. It also implies that the regulatory body failed to consider existing research or adequately investigate

Article 15: Numerous Congressional and internal investigations have called the oversight agency badly mismanaged and at times corrupt. It has been rocked by regular scandals, including disclosures in 2008 that agency officials took bribes and engaged in drug use and sex with oil industry officials. And its own scientists have said that senior agency officials in recent years revised staff reports to eliminate environmental concerns that might have complicated oil-company drilling applications for offshore sites in waters near Alaska. V.29 the potential effects of dispersants. This segment

demonstrates that although there is dominant

framing of many issues advanced by sources and further shaped by journalists, these frames are not all encompassing.

Other coverage focused on the MMS as a problematic institution staffed by flawed people (see box V.29). This particular article paints a condemning portrait of the corruption at MMS, including a 2008 scandal involving bribery and physical relationships between MMS regulators and

oil industry executives. A republican says that of the MMS: "There is a bureaucracy and dysfunctional culture that has to be held accountable" (Article 15). By discussing this "dysfunctional culture" after recounting tales of sexual relationships and drug use, the narrative further advances the frame that the MMS is staffed by problematic people, and if they were replaced, so too would be the dysfunctional culture. Throughout the spill coverage, discussion of problematic cultures only ever refer to the culture of risk at BP and the culture of negligence at the MMS, and never posit these as intersecting phenomena that could be linked to similar root causes. Although the willingness to 
discuss failure of government is a generally systemic critique, the discussion is still painted with the brushstroke of neoliberal individualism. Many Republicans have often betrayed that their motives are to disempower or outright dissolve the Minerals Management Service and Environmental Protection Agency; thus their critique of the 'dysfunctional culture' is likely aimed at shutting

Article 67: The panel also took federal offshore drilling regulators to task, for rubberstamping permits and proposed changes in well design, and for failing to adequately oversee operations on the rig. It said the Interior Department's Minerals Management Service (recently renamed the Bureau of Ocean Energy Management, Regulation and Enforcement) lacked the personnel, training and muscle to do its job and had essentially been captured by the industry it was meant to police. Box V.30 down the agencies rather than forcing them to regulate more effectively.

The coverage continues to focus narrowly on the failings at the MMS without discussing the government, which mandates and oversees their actions (see box V.30). Similar to BP, the MMS underwent a rebranding effort after the blowout in order to recoup their damaged public image (see box V.30). This article suggests that the MMS was improperly resourced rather than staffed by

problematic people. It likewise suggests that the industry essentially controlled the MMS, but does not explain how this went on unchecked. The Presidential Panel investigation faults the MMS for failing to regulate, but it does not ask who was responsible for overseeing the MMS, nor did it examine the political or economic environment that led to the problems. Although the lack of 'muscle' at the MMS is examined, there is no further investigation into how it ended up understaffed and presumably, underfunded. With all of the above examples in mind, it appears that the MMS is being scapegoated for a broader 
regulatory and government problem. The major response did not come in the form of stricter regulation, but a new name.

\section{Long Term Consequences}

As has been demonstrated throughout this chapter the environmental consequences in the Gulf are often overlooked, oversimplified, or framed only as a problem to industry and work. One article departs sharply from this norm and discusses the long lineage of problems in the Gulf of Mexico, explaining that the waters have suffered environmentally for a long time. The director of Florida State University's

Runoff and waste from cornfields, sewage plants, golf courses and oil-stained parking lots drain into the Mississippi River from vast swaths of the United States, and then flow down to the Gulf, creating a zone of lifeless water the size of Lake Ontario just off the coast of Louisiana. The Gulf's floor is littered with bombs, chemical weapons and other ordnance dumped in the middle of last century, even in areas busy with drilling, and miles outside of designated dumping zones, according to experts who work on deepwater hazard surveys. Box V.31
Costal Marine Laboratory proposes that the oil spill may be a deathblow to the Gulf rather than a solitary source of harm: "There's a tremendous amount of outrage with the oil spill, and rightfully so... but where's the outrage at the thousands and millions of little cuts we've made on a daily basis?"

(Article 48). The expert quoted in this article clearly suggests that the Gulf has been overlooked, but she does not clearly explain whether those cuts extend beyond environmental policy. The journalist elaborates later in the article: "But like no other American body of water, the Gulf bears the environmental consequences of the country's economic pursuits and appetites, including oil and corn" (Article 48). In many ways this passage encapsulates an otherwise absent discussion in the sample of how the long-term economic organization of the United 
States has caused severe harms in the Gulf. The journalist details the environmental stresses faced by the Gulf (see text box V.31). The discussion fails to extend beyond environment to the human costs, and explains the Gulf as a particular body of water that has been neglected by the environmental movement and suffered as America grew.

The director of an environmental group suggests: "This has been the nation's sacrifice zone, and has been for 50-plus years... what we're seeing right now with BP's crude is just a very photogenic representation of that" (Article 48). This passage reinforces the notion that the Gulf is an isolated and abandoned section of ocean that has been victimized by economic pursuits and appetites, rather than one of many struggling environments reeling from policy that empowered industry and government alike to prioritize profit over the environment. The article does speak to the need for tougher enforcement of regulation. This conversation still requires imagination on behalf of the reader to expand the environmental consequences of American industrialization beyond the Gulf or to foresee possible regulatory solutions. The article suggests that resistance comes from citizens of the Gulf states themselves, but the reality may be that the pressure comes from state governments who are closely tied to the oil industry. This article is one of the most critical and the only one that clearly links the Gulf to an economic history. Even with this critical opening, there is no discussion of the broader political forces that led to the state of the Gulf.

This article critiques the lack of regulation, but likewise accepts the neoliberal paradigm that special interest groups and charities should take the place of large-scale government oversight: "But without the aggressive watchdog role played by wellfinanced environmental groups in places like California and the Mid-Atlantic. threats to 
the Gulf have largely gone unmonitored" (Article 48). This article suggests that environmental groups have not traditionally had strong footing in the Gulf; the area has only had the attention of oil and gas lobbying. The article even cites "several national environmental groups" as suggesting that the Gulf is a hostile site for environmental fundraising and awareness campaigns. Effectively, this discourse places blame for the spill on a lack of ambition in environmental groups and an unwilling local population. This article reinforces the importance of local political economic context and suggests that locals believe in the importance of the oil industry in the Gulf.

The sample omits a broad conversation about the potential long-term environmental and human impacts. Although it may seem challenging to integrate these discussions into the news, within a single brief Greenpeace press release (Greenpeace 2010) the head of their energy campaign proposes a moratorium on deepwater drilling and relates the incident to other proposed deepwater wells. He argues that the root cause of the spill is addiction to oil, which he blames for the risky behavior of oil companies. He does not directly focus blame on BP, but targets governments for not stopping or controlling environmental exploitation in the Gulf, Arctic and Alberta tar sands. The press release suggests that investing in new sustainable energy and reducing energy demands are the only real solutions to the approaching end of oil. Aside from the Governor of Florida who suggested a permanent moratorium on deepwater wells, nowhere in the sample is the industry framed as inherently problematic as it is by environmentalists at Greenpeace. I also looked at a press release by BP that summarized the findings of their internal report. Unlike the press release by Greenpeace, the BP press release was featured prominently in the sample as a component of the day's news. There 
was never an environmentalist report or press release cited; Greenpeace, an environmental group with national recognition, has access in a single article, as was discussed in Chapter Four.

Some articles in the sample described the spill as a potential environmental disaster, particularly as the flow estimate continued to rise and capping procedures continued to fail. Reactions focused on how many animals had died, how many more were expected to die, and what the potential impacts on the industry were. This discussion is doubtless important, but fails to tackle the political realm or human impacts. The sample does not discuss the long term effects of oil spills in other communities. One might expect it to include some discussion of Prince William Sound, where rates of depression and suicide in the affected communities are still elevated over 30 years after Exxon Valdez ran aground (Joling and Thiessen 2010).

Article 51 discusses the anxieties of Gulf residents and for the first time brings up long-term human impacts. The article entertains the notions that the spill could have health and psychological affects on residents for years that go beyond financial: "The more confrontational tone from Washington underscored concerns within the administration about the long-term effect of the oil spill, not only environmentally, economically and politically, but on the national psyche as well" (Article 51). Aside from one other article where BP denies that they will pay for claims around mental health, there is no explicit discussion on long-term health of the community beyond the stress of unemployment and reduced wages. As was discussed more explicitly in the previous data analysis chapter, focus only on work and labour prioritizes the economy above all other social concerns. 


\section{A Likely Accident}

The spill was almost always painted as an unlikely accident in the sample. but there can be little doubt that some form of major oil spill was bound to occur in the Gulf, and given that no legislation passed to regulate deepwater drilling at the time of this writing, one will likely occur again. The spill is occasionally suggested to have been a likely outcome, but the pieces are never assembled in a clear coherent way that describes the similarities between the operating practices of $\mathrm{BP}$ and other drilling companies.

When viewed as a part of a whole, the isolated

Article 27: In April of this year, $\mathrm{BP}$ engineers concluded that the casing was "unlikely to be a successful cement job." The document also says that the plan for casing the well is "unable to fulfill M.M.S. regulations," referring to the Minerals Management Service. A second version of the same document says, "It is possible to obtain a successful cement job" and "It is possible to fulfill M.M.S. regulations.

Box V.32 segments suggest there were human, equipment or industrial flaws leading up to the spill. They contradict the frames that the rig was sophisticated, safe and that its explosion was a fluke.

Some internal BP documentation from before the spill demonstrates that the rig was bound to face a blowout, although the journalist never explicitly says this (see box V.32). BP was not only in violation of their own safety policies, but those

of the MMS. BP was knowingly in defiance of regulations and was able to adhere to regulations but opted not to; they were even aware that the cement job was risky and likely to fail. This article blames both BP and federal corporate oversight and explains that BP had numerous safety concerns and problems with the Deepwater Horizon drilling rig right up to the explosion. An internal $\mathrm{BP}$ document revealed that some safety procedures were being deliberately disobeyed. 
Other experts even agreed that the scenario

looked unsafe and that it was likely that BP was

"facing a blowout scenario" (article 27). Other

politicians warned of the possibility for repeat

spills. Bob Graham suggests: "If dramatic steps

are not taken I' $m$ afraid at some point in the

coming years another failure will occur, and we

will wonder why did the Congress, why did the

administration, why did the industry allow this to

happen again" (Article 70).

Article 67: Offshore oil exploration is by nature risky, the commission concluded. "Notwithstanding these inherent risks, the accident of April 20 was avoidable," the panel wrote. "It resulted from clear mistakes made in the first instance by BP, Halliburton and Transocean, and by government officials who, relying too much on industry's assertions of the safety of their operations, failed to create and apply a program of regulatory oversight that would have properly minimized the risk of deepwater drilling."

Box V.33

Article 56 suggests that $54 \%$ of Deepwater

Horizon employees were afraid to bring up problems on the rig for fear of repercussions.

Immediately after the spill, several articles had suggested that the rig was safe, ahead of

schedule and unproblematic. A quote from the

Article 67: The commission warned that without major immediate reaction to the spill had even suggested changes, another such accident was likely. "The blowout was not the product of a series of aberrational decisions made by rogue industry or government officials that could not have been anticipated or expected to occur again," it concluded.

"Rather, the root causes are systemic and, absent significant reform in both industry practices and government policies, might well recur."

Box V.34

that the MMS had recently inspected the rig and it

had been up to code (Article 2). This was revealed to

be untrue later, but at a time when the media

coverage of the spill was less dense. The initial

framing suggested that the rig suffered a freak

accident and this idea may have stayed with the

readers throughout the progression of the spill.

One of the final articles in the sample 
discussed the findings of the presidential panel, and tempered outright criticism with the acknowledgement that the job is inherently risky (see box V.33). Despite the authority with which the presidential panel declared the accident preventable, it also describes errors only as mistakes rather than part of a trend of negligence. This choice of vocabulary is significant since the fines and repercussions BP faces increase dramatically if the company is found criminally negligent. Although the article begins by suggesting that the accident was the result of a series of "failures and blunders, it eventually covered more systemic issues.

This segment in box V.34 from the Presidential Panel report is possibly the most critical look at the reasons for the spill included in the sample. However, the segment does not shape the tone of the overall article, which quickly goes back to focusing on the three companies who share blame for the event. The report also adopts the notion that the industry can be easily reformed rather than looking to the broader corporate and government cultures that led to the accident. Noticeably absent from the report was discussion of the potential repercussions for the CEOs and executives of the companies. No legislation followed this report (Freudenburg and Gramling 2011).

\section{The Role of Industry and the Economy}

As discussed in the previous section, the sample evidenced that there were many indicators a blowout was likely to occur. There were also some indications that this problem extended beyond $\mathrm{BP}$, but this frame needed to compete with the much more prevalent suggestions that BP is a corporate bad apple and the spill was a fluke. Thus, blame was seldom directed at the oil industry. The industry was alluded to as a problem by the Presidential Panel's report, but this critique is brief and quickly shifts back to the 
problematic actors. Senator Patty Murray departs from the norm and blames the industry rather than focusing only on BP. She was quoted responding to a series of confidential. internal Transocean reports (see box V.35). This senator is a lone voice in the sample that bluntly criticizes the entire oil and gas industry and explains that it should not benefit from similar protections that are given to small business. However, the reoccurring frame of article 56 is that Transocean is a problematic company, not the industry on the whole.

Transocean responded positively to the same internal reports and responded proudly to the $87 \%$ of workers who felt they had time to complete their work safely and the seven years without a major incident on the deepwater horizon.

Article 56: "These documents are more evidence that despite the growing count of worker deaths and safety violations, the oil and gas industry still just doesn't get it," she said. "They need to change their workersafety culture, and I am pretty sure we can't count on them to do it by themselves." She added, "The oil and gas industry is not the same as a mom-and-pop grocery, and they can't be treated the same." Box V.35

Transocean does not find it problematic that $13 \%$ of rig workers do not feel safe completing their work, and they suggest that seven years of safety is worth bragging rights even though it ended abruptly with the eleven deaths, a number of serious injuries and the Deepwater Horizon on the seafloor.

Generally, the oil and gas industry was not targeted as a source of blame and instead the discourses normalized the frame that offshore oil is a crucial domestic source of energy and jobs and reform (if any) must be focused on the few problematic institutions. These frames justify the use of risky sources of deep water oil that jeopardizes delicate coast line and sea life. Paradoxically, every administration since Richard Nixon's has called for American energy independence, while the percentage of 
oil that is imported increases steadily (Freudenburg and Gramling 2011). However, there is a near unanimous scientific consensus that the United States will never find enough oil in its territory to sustain anywhere near the current levels of demand.

Article 23 comes closer than the rest of the sample to suggesting there are broader forces that have contributed to the current situation in the Gulf. "Only a handful of such ships supply the Gulf of Mexico, and the best-outfitted boats tend to work for the oil industry. Exploring and protecting the Gulf has simply not been as high a national priority as drilling it for oil." The brief segment suggests that the United States has prioritized oil drilling over the health of the Gulf environment. The implication is that more funding and attention should go to scientific exploration of the Gulf, but the article does not question the framing of drilling as a national priority, and only problematizes the failure to prioritize the health of the Gulf waters. Very few articles discuss the economic organization of the United States, or trends in the global economy. Although this may be a daunting task for many event-focused articles with limited space, there are no articles that clearly link the pressures faced by Gulf residents, regulatory bodies, and the industry itself into a broader narrative about the accelerated economic expansion of the United States. Although specific companies carry out the drilling, it is only through the channels of the US government that they are empowered to drill. One article suggested that the MMS 'rubber stamps' drilling permits (Article 67), which could arguably be connected to drilling as an official national priority to reduce foreign dependence on oil by increasing the domestic supply.

Article 68 explains that the lives of fishermen are increasingly difficult as more American seafood is provided by Asian fish farms and shipped in from overseas. The 
article focuses predominantly on the struggles faced by a single family and explains that the father earned more per pound 19 years earlier when his son was born than he does now. The article does not explore why the economy has changed to disadvantage domestic fisheries but places some abstract blame on the global economy, which is presented as an unavoidable phenomenon. Discussion that links to the broader economy is subtle and never liked to a global economic macrostructure that will likely continue to affect lives and work in a similar way.

There are many quoted sources in the sample who promote a business solution to the spill, and suggest that reacting with increased industry-wide regulation would be a mistake. Alternately, Article 26 subtly implies that the journalist is skeptical of the extreme pro-business views presented by the sources in the article, but it is never made explicit. The article never even opens up to views on the opposite end of the political spectrum. Although the journalist may be unconvinced by the business rhetoric that is provided by Hailey Barbour and Rick Perry, there is no space allotted for alternate views. The converse to neoliberal pro-business is generally absent in the mainstream U.S. political spectrum. The article could have given equal attention to environmentalists or academics who are critical of the global economic status quo. Although there may not have been space to give adequate attention to these voices within an article on the state governors, there is no comparable article in the sample that gives similar attention to dissenting voices. Right wing voices that prioritize business are prevalent in the sample, but any critique from the left is scattered and never presented as an organized system of ideas. As has been discussed in great detail above, the criticism of the spill generally focuses on $\mathrm{BP}$ and proactive solution about how to fix the company and the 
environmental damage rather than what broad changes are required to fix the industry, government and economy.

\subsection{Conclusion}

I began this project with the observation that the spill coverage appeared to present complex social and political problems as individual issues of blame and responsibility. As I coded for the frames presented by sources and journalists, frames did begin to cluster around individualization, but also the tendency to minimize the size and scope of the spill, to promote business solutions to the spill while always prioritizing continued economic growth, and to omit challengers to these themes. The observations and quotations contained within those themes emerged organically from the data to paint a clear portrait that the frames in the sample reproduces neoliberal ideology. The most prominent symptom of neoliberal narrative in the sample is that the coverage simultaneously proposes narrow individual answers to social problems while giving space to the narrative that the spill was an act of god, a technical problem, the result of human error, and the consequence of deviant corporations. These observations would be significant on their own, but are particularly striking when viewed in concert with the final section of this paper, which demonstrates that similar space is not allocated to systemic discussion that is non-neoliberal.

This chapter demonstrates an example of a disproportionate focus on free market economic solutions to industrial environmental accidents. The sources that promote this frame prioritize business above all other social concerns and that suggest that corporations should self regulate. Many of the same sources suggest that drilling should never be interrupted and that regulation will have negative impacts on the economy. This 
discourse promotes non-government solutions to the problems proposed by the spill and thus advances the ideological neoliberal argument that the government should be focused primarily on ensuring proper conditions for the economy rather than providing social protection to its people. As was demonstrated in Chapter Four, locals and other sources are often very worried about the spill, but these concerns are seldom connected to broader systemic problems that have led to the spill and which will likely continue to lead to environmental disasters. This construction of the local voice allows for the advancement of pro-business sources who suggest the media is exaggerating the spill and that the environmental consequences will be small. Although there are some openings for voices that propose the spill is much larger than anticipated, these sources are seldom quoted or given space for a technical explanation of the potential parameters and environmental consequences. This conversation demonstrates clearly the linkage between Chapters Four and Five: the construction, portrayal and access granted to different sources shape the subsequent frames and broader framing packages, which generally advance neoliberal ideology.

The articles included in the sample focus prominently on solutions to the spill rather than understanding the underlying causes, which obscures blame and avoids systemic discussion of trends in many economies worldwide. Although some voices blame a lack of government regulation, this failure is not connected to politically motivated changes since the era of Ronald Reagan to reduce the size and scope of government while allowing industries to grow limitlessly with low taxation in an almost total absence of regulation. Critical voices, which would look to disseminate long-term consequences, are omitted, and the various threads that suggest the spill was nearly an 
inevitable consequence of a faltering system are not pieced together, but scattered throughout the coverage. Thus, it would be a mistake to suggest the spill coverage uniformly and homogeneously supports neoliberal ideology, but the organization and framing of the spectrum of attitudes has led the argument irrefutably toward this conclusion. 


\section{CHAPTER VI}

\section{Conclusion}

"If we've learned anything so far about the deepwater Gulf of Mexico, it is that it contains surprises. And that means an operator needs depth-depth in terms of resources and expertise - to create the capability to respond to the unexpected" (article 28).

-David Eyton in 2005, then VP of BPs deepwater developments in the Gulf of Mexico,

The former vice-president of BP's deepwater operations in the Gulf spoke prophetically about the depth of working in the water; the physical depth and the depth of knowledge required to deal with the unique challenges in the Gulf. His speech was for an oil and gas industry crowd and was thus optimistic about BP's capacities to work at these depths. However, he also cautioned against the unbridled optimism typical of the industry and subtly suggested that technological progress must be tempered with reason. Words similar to these are unlikely to be spoken publicly by a BP representative again, at the very least until the legal ramifications of the spill are settled. BP failed to exercise depth beyond the physical depth of their drilling. The company failed to heed the advice of the executive who had been in charge of deepwater operations. BP's safety lacked depth and foresight, and eventually led to a deadly and destructive blowout.

The frames presented in the news coverage of the blowout in the New York Times seldom reflected depth nor did they tell a cautionary tale, such as those presented by the BP President in 2005. Instead, sources often focused narrowly on the technical causes of the spill and the immediate financial repercussions. Although I did not set out specifically to detect neoliberal ideology, I have concluded that many of the included frames clearly reproduce the thinking and priorities of neoliberalism and promote the 
corporate status quo. Prominent sources downplay the magnitude of the spill and focus on business solutions. Although there are some outliers and challengers, generally the media analysis presented here reveals a critique that blames individuals and specific corporations without connecting their practices to economic policy or the historical political decisions that have led to the status quo. Potential human, cultural, and longterm impacts are obscured and the magnitude of the spill is minimized.

This project draws heavily on Molotch and Lester's (1975) investigation of the Santa Barbara oil leak. Their study of the 1969 Union Oil subsea leak off the California coast served as a crucial case that set the tone for my own research. As the project developed and I interacted with the data and literature, it became increasingly clear that the construction and portrayal of industrial environmental accidents in the news continues to be a valid site for investigation.

My study differs from Molotch and Lester's (1975) in several key ways. First, they found that there were two large generally opposing groups: the government who sided with Union Oil, and locals who sided with conservationists. On the other hand, my contemporary New York Times data quickly revealed that there were few similarities between the local and environmentalist voice, which never rallied together against BP. Environmental groups and conservationists were infrequently mentioned in the data. While the local voice had a high quantity of coverage, even these voices often evidenced neoliberal ideology.

Second, the culture and economic history of the southern Gulf States is dramatically different than coastal California. The Gulf States (aside from Florida, whose politicians took a more conservationist stance) have depended heavily on oil and fishing 
revenue and jobs for generations. While fishermen seldom go on to become powerful state governors, many Gulf politicians have strong ties to the oil industry. Voices such as Hailey Barbour and Rick Perry spoke bluntly on behalf of the oil industry. Molotch and Lester even found in their study that there was little coverage in the "coastal oil-oriented city of New Orleans" (1975: 243), and prophetically noted that Santa Barbara's oil troubles should have been taken seriously in the Gulf press since the area is at high risk for spills.

Third, there have been dramatic economic and cultural changes between 1975 and 2011 both within the United States, and globally. I focused specifically on the rise of neoliberal economic policy and the erosion of regulation and social protections. Molotch and Lester's (1975) study was indeed groundbreaking, and this same style of investigation provides key insights into large-scale differences over geography and time and how these affect the construction of an event in the news. The two cases seem to be dramatically different primarily due to cultural and economic changes over 40 years and the different political economic histories of California and the Gulf coast. While Molotch and Lester's study was an appropriate starting point for analysis of the role of media in the construction of environmental events, this research area should be continually revisited as the economy and culture change, particularly in different political economic contexts. Finally, Molotch and Lester (1975) suggest that there is no objective reality to be known. As has been demonstrated in the thesis, the project is grounded in the belief that there is a material reality that affects both journalists and people.

My research demonstrates a tendency in media to explain broad social problems in individual terms. While this observation can be clearly linked to the influence and 
reproduction of neoliberalism, it is a significant finding on its own. The tendency to focus on individuals and problems with a limited scope significantly impairs the quality and depth of material presented in news coverage. Examples in the sample often suggest that the spill is a technical problem rather than a broader social problem. To counteract this trend, the media could seek out voices who suggest that the disaster is at its heart an industry problem, a problem with the way the economy is structured, or a problem of the high demand for fossil fuel. On almost every level, this research has reflected a failure by media to connect with systemic critique in a consistent or organized way. While structural solutions were present, they almost unanimously suggested that business should self regulate. Alternate narratives on how to move forward from the spill were minimized. Once these observations began to take shape, it was clear that my argument broadly needed to encapsulate the presence of neoliberal ideologies in the spill. Strands and packages of neoliberal ideology in the coverage were not unified. but were unavoidably prominent.

This study demonstrated the tendency to adhere to the objectivity paradigm in news coverage. That is, journalists did not clearly advance their own frames, but participated in framing by choosing which source frames to include, how to portray them, and how often. Journalists default to quotations from sources to tell the story instead of defending a version of events. Likewise, journalists to relied on authoritative sources and cited these sources disproportionately, especially at the beginning of the articles. These findings support prior literature, which nearly unanimously suggests journalists rely heavily on official channels for sourcing in an effort to be objective (Carlson 2009; Molotch and Lester 1975, Anderson 1999 and 2002; Schudson 1989). This project 
contributes by illustrating the continuation of this trend through a particular example of media analysis around a contemporary case of an industrial environmental accident.

As confirmed by nearly all research on the media, the role of the media is one that is evolving, particularly with the rise of social media and the increasingly-up-to-theminute style of reporting (Carlson 2009; Anderson 2002; Schudson 1989). My data cannot on its own reveal historical changes in the overall direction of media given its limited parameters. However, I suspect that members of the media are under increasing pressure with fewer resources, similar to many contemporary professionals. This pressure reveals itself in the adherence to trusted, authoritative and bureaucratically organized sources, which legitimates the status quo of authority and relegates local voices to narrowly defined human interest pieces. Rather than explicitly participating in an ongoing debate on whether the constraints on media are affecting the practice of journalism, or conversely if corporate ownership affects the quality of news, I see these trends as intimately connected. As I have demonstrated, the neoliberal turn in the economy has led to the conditions that created the spill through a push for increased profit, reduced government oversight, and the shared interests of governments, regulatory bodies and corporations. Likewise, this push for competition may be forcing journalists to work on reduced budgets. Neoliberalism promotes a single market ethic over all other social and human values; in order to function as a watchdog to corporations and governments, journalism must prioritize the public good and the responsibility of the press to investigate and improve public awareness of major events such as an environmental disaster in an ecological commons. These values are antithetical to the priorities of neoliberalism. 
Journalism may be suffering under the same set of economic objectives that contributed to the spill because neoliberal economic organization contributes to constraints such as the pressures of the 24-hour news cycle and over-reliance on expert sources. By almost all accounts in the literature and findings of this project, the products of the media focus on the short term, are not in-depth, rely on authoritative sources, and trivialize and depoliticize other voices. All of these consequences can be seen as at least partially as the result of neoliberal economic values on the broader culture. The tendency to prioritize neoliberal frames in media means that an increasing array of the discourse we all see, read, and hear suggest business solutions to problems. This study represents an extreme case; the New York Times is an agenda setting paper in the United States and comes closer to the left of the political spectrum than any of its other mainstream peers. Despite these features of the New York Times, neoliberal framing still permeates the media portrayal of the spill and prioritizes business interest over human interest. Although it is hard to find evidence that the media is directly controlled by the strong hand of business, neoliberal ideology is clearly evidenced at nearly every level of the study, and may be affecting the organization of corporations and newsrooms in very similar ways.

One of the more surprising findings in the study was the prominence and character of the local voice. However, this finding reinforced the importance of a nuanced investigation into the coverage. Locals represented a more diverse group of voices than any other single category. However, these voices still often reproduce a neoliberal ideology, and while mourning for environment loss, fail to take the devastation beyond the realm of the emotional. This trivializes the local voices as sites of "human 
interest" rather than as actors who could contribute to a political critique of the economy and government. As was mentioned above. this is a key point of departure from Molotch and Lester's (1975) study. Beginning this research and considering previous literatures, I would have expected the local voice to be less frequent, but more critical of BP. Instead, locals are frequently quoted but reproduce many dominant frames from sources such as BP, politicians and expert sources. This finding may indicate that neoliberal ideology has permeated the common sense of mainstream media institutions and journalists.

My study fits with the view that media studies should, and necessarily do, investigate imprints of power (Entman 2002, 2007). Power is exercised in knowledge production, which most often reproduces the status quo. This power lies in the capacity to create and design public events (Molotch and Lester 1975). Studying the access to media is fundamentally a study of power relationships. As demonstrated by Carlson (2009) debates in the literature emerge over who is ultimately wielding power: the sources, the journalists, or both? However, reductive answers are seldom informative. Sociological media investigations instead track how this imprint of power shifts. Power struggles define the relationships between various competing sources and actors. The nature of the event shapes the power dynamic, while the contests within this dynamic simultaneously shape the event. This thesis demonstrates that media often lack the capacity to connect events to their social, cultural and political context due to institutional constraints.

The media coverage focused on economic impacts and solutions to the stark neglect of long-term environmental or human consequences. This financialization of everything is a prominent trend in the impact of neoliberalism (Fairclough 2001). Even environmental problems are framed in economic terms. Politicians, executive and experts 
all called for Americans to prioritize industry and jobs rather than environment or industry regulation. Ordinary citizens who live and work on the Gulf repeated this financial frame. The tendency to reduce every component piece of social life to economic terms marginalizes voices who have limited participation in the economy. This was also reflected in the tendency only to interview affected Gulf workers, while almost entirely ignoring general residents.

When considering the implications of this research, I return to Flyvbjerg (2001) to ask: where are we going and is it desirable? My investigation looks at a particular extreme case of environmental degradation and its coverage in the media. What does the investigation of media responses indicate about where we are headed, specifically where are the media headed and how should they continue to act? It is possible that catastrophes such as the BP spill will become more common as the desire for oil increases with a growing and industrializing worldwide population and drilling goes into more dangerous and isolated oil wells. Careful investigation of how spills are handled may become increasingly relevant. The causes and long-term consequences of the recent BP spill are still the subject of debate; this project is among the first in-depth investigations into the social impacts of the Deepwater Horizon explosion.

This study contributes to ongoing political economic debates on the balance of power between governments and large corporations, the deepening of neoliberalism, and the evolving roles of the media and of environmentalists in these questions. Thus, my research has theoretical implications for the economic organization of many nations that subscribe to neoliberal economic ideology. While the US serves as the template for 
intensification of corporate power in government, countries like Canada follow closely behind (Keil 2002: Mulvale 2001; Carroll and Shaw 2001).

Molotch and Lester (1975) found that openings in the status quo of journalism may be granted due to the 'accidental' nature of oil spill news. Although the focus of my research was not on a temporal comparison of varying coverage along the progression of the spill, the tendency to challenge the status quo was rare, and especially uncommon in the early days of the spill, which focused on technical details and praised the technological sophistication of the rig. Does the sudden and unexpected nature of an oil spill still provide an opening for access by unconventional, "outsider" sources? My research would suggest this may no longer be true, possibly due to the preparedness of large corporations for an accident, their focus on public relations, and the increasing power of large corporations in politics. Thus, future researchers could explore to what extent the accidental nature of an industrial environmental disaster still provides the access described by Molotch and Lester (1975).

This study provides a commentary on environmental issues in media. Critique of the media's failure to discuss the neoliberal deregulatory history in the United can be tied to the question of the ongoing efforts by corporate and economic elites to reduce legal restrictions on the extraction of natural resources from ecological commons and the partnership of the state in this process. My research indicates that corporate and government frames are nearly indecipherable in the media coverage, both of which prioritize the economic status quo,

At its core, this thesis has enabled me to investigate the extent to which (and how) neoliberal economic ideology and its inherent assumptions are evidenced in the print 
news response to an environmental disaster. This project leaves many openings for continued research. Prominently, this media work could be combined with interview data from Gulf residents and members of the media. This would enable the researcher to explore the news values that guide the media and compare the portrayal of Gulf residents' reactions in media to their actual perceptions of the spill. Further research could also extend the time period studied; the legal and financial liability for the spill is scheduled to be settled in a New Orleans court in February 2012. Just as the environmental, human and legal consequences are as yet unknown, the spill and its portrayal in media will continue to evolve. As global populations increase and industrialize while scarce resources disappear, environmental catastrophes will continue to occur, particularly in a political and economic climate that prioritizes growth and profit over stability and quality of life. Continued investigation into the media responses to these events will provide insight into both the media itself, and the way the public understands their institutions, governments, and their impacts on the planet. 


\section{Appendix A}

\section{Table One: Total Quoted}

Articles 1-17

$\begin{array}{lllllllllllllllll}1.1 & 1.2 & 1.3 & 1.4 & 2.5 & 2.6 & 2.7 & 2.8 & 2.9 & 2.1 & 2.11 & 2.12 & 2.13 & 2.14 & 2.15 & 2.16 & 2.17\end{array}$

Locals

Fishermen

General Locais

Local Politicians

$\begin{array}{llll}1.22 \quad 1.3 & 1.4 \quad 2.5 \quad 26 \quad 27\end{array}$

Local Businesses/Business owners

Rig Worker

$\begin{array}{llllll}1 & 3 & 1 & & & 1 \\ & & & 3\end{array}$

5

2

Local Attomey

Other (non-profit, land trust, other

officrals)

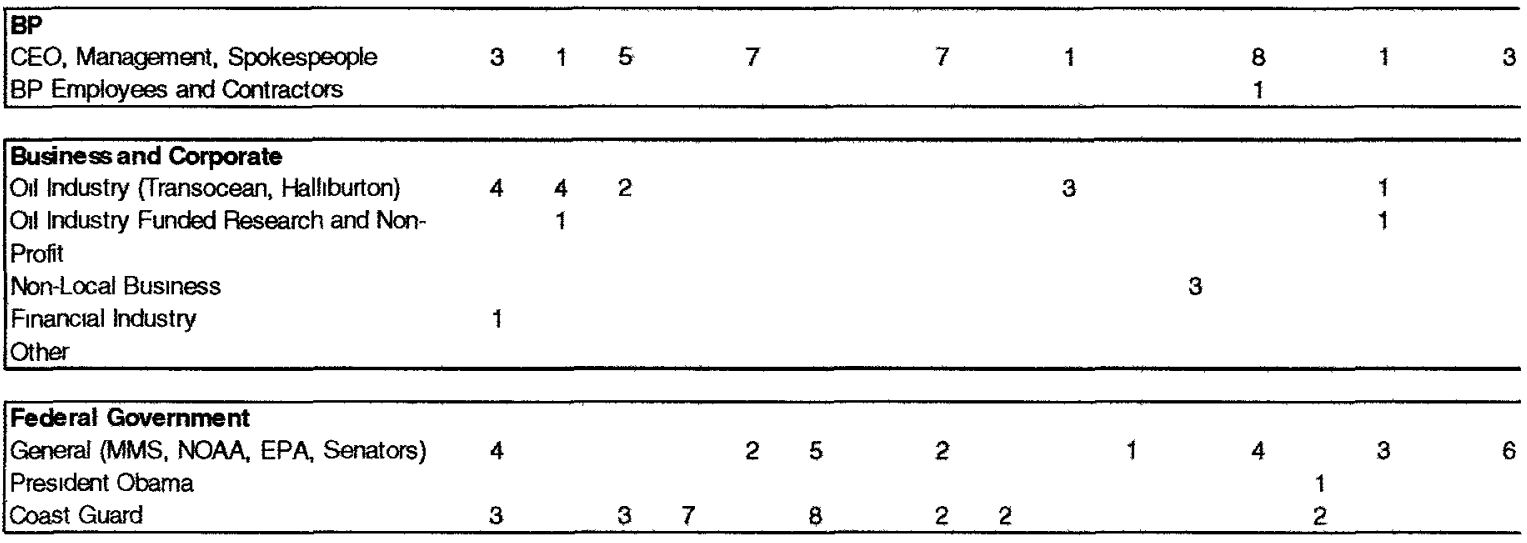

BP and Govemment (quoted as one)

Anti-BP Sources

Anonymous Employees

Condemning documents

1

\begin{tabular}{|c|c|c|c|c|c|c|c|c|c|c|c|c|c|c|c|c|}
\hline $\begin{array}{l}\text { Experts (Non-BP) } \\
\text { Scientist } \\
\text { Engineer } \\
\text { Brologist } \\
\text { Academic (non-oll Industry) } \\
\text { Energy/Petroleum Academic } \\
\text { Other }\end{array}$ & & & & 5 & & & & & & 4 & 1 & $\begin{array}{l}1 \\
1 \\
1\end{array}$ & & & & \\
\hline Environmentalists & & & & & & & 2 & & & 2 & & & & & & \\
\hline $\begin{array}{l}\text { Other and Unattributable } \\
\text { Unattnbutable 'Analysts' and 'Officrals } \\
\text { General Other }\end{array}$ & & & & & 1 & & & & & & & & & & 2 & \\
\hline $\begin{array}{l}\text { First Quoted } \\
\text { Local } \\
\text { BP } \\
\text { Business and Comporate } \\
\text { Federal Govemment } \\
\text { Enuronmentalists } \\
\text { Experts (Non-BP) } \\
\text { Other and Unattnbutable } \\
\text { Ant1-BP Sources }\end{array}$ & 1 & 1 & 1 & 1 & 1 & 1 & 1 & 1 & 1 & 1 & 1 & 1 & 1 & 1 & 1 & 1 \\
\hline
\end{tabular}




\section{Articles $18-35$}

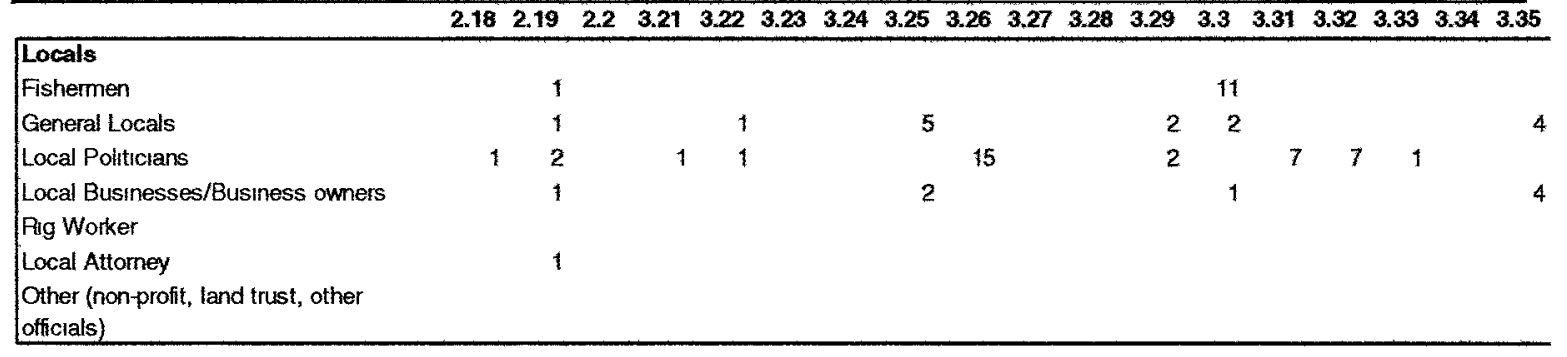

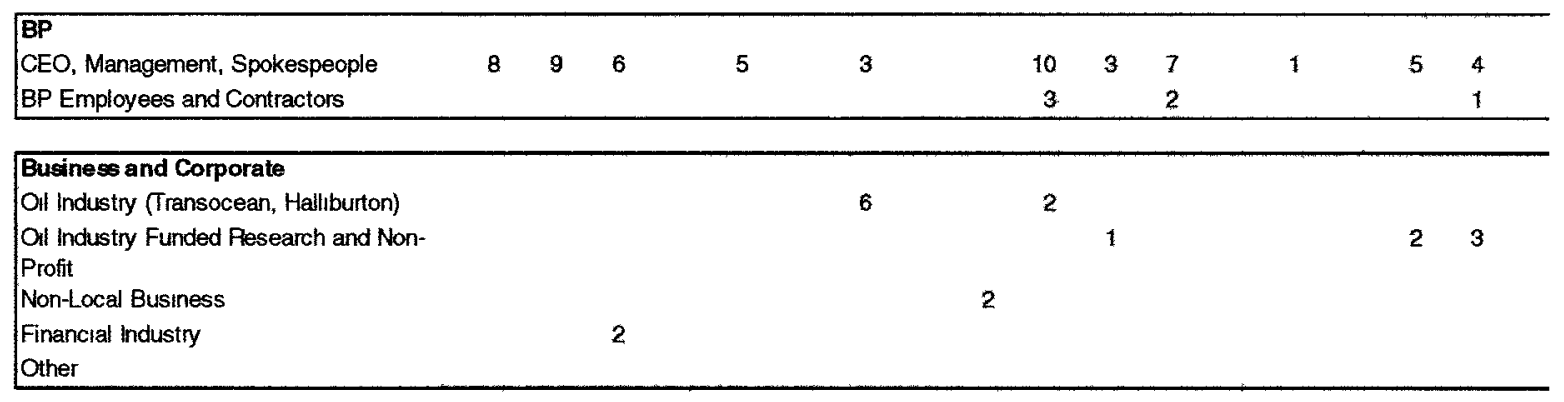

Federal Government

General (MMS, NOAA, EPA, Senators)

President Obama

Coast Guard

4

$\begin{array}{llll}11 & 6 & 1 & 1 \\ 1 & 1 & & \end{array}$

BP and Govemment (quoted as one) 11

\begin{tabular}{|c|c|c|c|}
\hline \multicolumn{4}{|l|}{ Antr-BP Sources } \\
\hline Anonymous Employees & 5 & 3 & 1 \\
\hline Condemning documents & 3 & & \\
\hline
\end{tabular}

\begin{tabular}{|c|c|c|c|c|c|c|c|c|c|c|c|c|c|c|c|c|c|c|}
\hline \multicolumn{19}{|l|}{ Experts (Non-BP) } \\
\hline Scientist & & & & & & 3 & & & & & 9 & & & 1 & & & 1 & \\
\hline Engineer & & & & & & & & & & 2 & & & & & 1 & & & \\
\hline Biologist & & & & & & & & & & & & & 2 & & & & & \\
\hline Academic (non-ol industry) & & & & & & & & & 1 & & & & & & & & & \\
\hline Energy/Petroleum Academic & & & 1 & & & & & & & & & & & & & & & \\
\hline \multicolumn{19}{|l|}{ Other } \\
\hline Environmentalists & & & & & & & & & 2 & & 3 & & & 8 & & & & \\
\hline \multicolumn{19}{|l|}{ Other and Unattributable } \\
\hline Unattributable 'Analysts' and 'Officials & 4 & & & & $\mathfrak{1}$ & & & & & & & & & & & & 2 & \\
\hline General Other & 1 & & & & & & 6 & & 1 & & & & & & & & 1 & \\
\hline \multicolumn{19}{|l|}{ First Quoted } \\
\hline Local & & 1 & & & & & & 1 & 1 & & & & 1 & & 1 & & & 1 \\
\hline $\mathrm{BP}$ & & & 1 & & & & & & & 1 & 1 & 1 & & & & & & \\
\hline Busmess and Corporate & 1 & & & & & & & & & & & & & & & & & \\
\hline Federal Govemment & & & & 1 & & & & & & & & & & & & 1 & 1 & \\
\hline Enuronmentahsts & & & & & & & & & & & & & & 1 & & & & \\
\hline Experts (Non-BP) & & & & & & 1 & & & & & & & & & & & & \\
\hline Other and Unattnbutable & & & & & 1 & & 1 & & & & & & & & & & & \\
\hline Anti-BP Sources & & & 1 & & & & & & & & & & & & & & & \\
\hline
\end{tabular}


Articles 36-53

$\begin{array}{lllllllllllllllllll}3.36 & 3.37 & 3.38 & 3.39 & 4.4 & 4.41 & 4.42 & 4.43 & 4.44 & 4.45 & 4.46 & 4.47 & 4.48 & 5.49 & 5.5 & 5.51 & 5.52 & 5.53\end{array}$

Locals

Fishermen

General Locals

Local Politicians

$5 \quad 2$

Local Businesses/Business owners

Pig Worker

Local Attorney

Other (non-profit, land trust, other

officials)

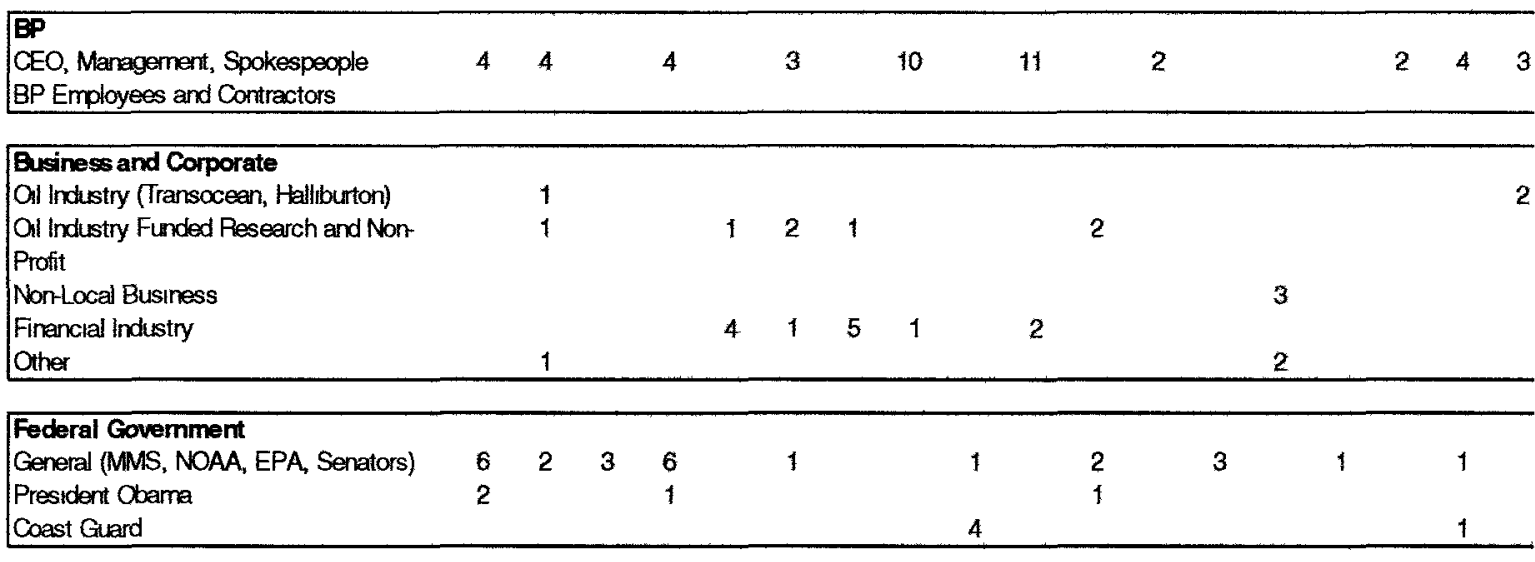

\begin{tabular}{|lll|l|l|l|l}
\hline BP and Government (quoted as one) & 1 & 1 & 1 & 1
\end{tabular}

Anti-EP Sources

Anonymous Employees

Conderming documents 11

\begin{tabular}{|c|c|c|c|c|c|c|c|c|c|c|c|c|c|c|c|c|}
\hline $\begin{array}{l}\text { Experts (Non-BP) } \\
\text { Sclentist } \\
\text { Engineer } \\
\text { Blologist } \\
\text { Academuc (non-ol Industry) } \\
\text { Energy/Petroleum Academic } \\
\text { Other }\end{array}$ & & 1 & 6 & & & 1 & & 1 & 2 & & & & 3 & & 2 & 2 \\
\hline Environmentalists & & & 1 & & 1 & & 1 & & 3 & & & & 3 & & & \\
\hline $\begin{array}{l}\text { Other and Unattributable } \\
\text { Unattnbutable 'Analysts' and 'Officlals } \\
\text { General Other }\end{array}$ & 3 & 1 & & 1 & & & & & & & & & $\begin{array}{l}1 \\
2\end{array}$ & & & \\
\hline $\begin{array}{l}\text { First Quoted } \\
\text { Local } \\
\text { BP } \\
\text { Business and Corporate } \\
\text { Federal Government } \\
\text { Enuronmentalists } \\
\text { Experts (Non-BP) } \\
\text { Other and Unattnbutable } \\
\text { Antr-BP Sources }\end{array}$ & 1 & 1 & 1 & 1 & 1 & 1 & 1 & 1 & 1 & 1 & 1 & 1 & 1 & 1 & 1 & 1 \\
\hline
\end{tabular}


Articles 54-70

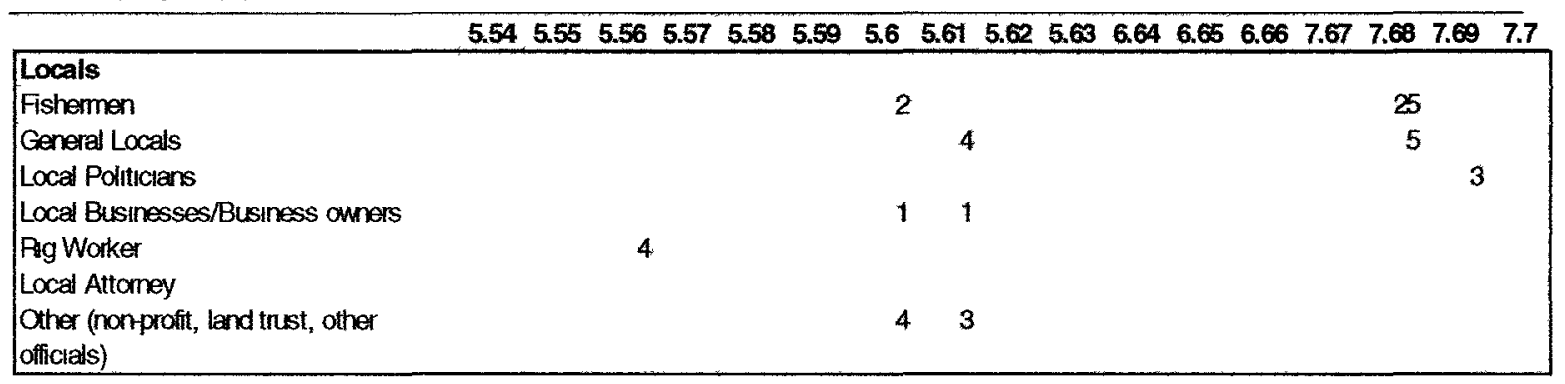

\begin{tabular}{|llllllllll}
\hline BP & 3 & 2 & 1 & 1 & 2 & 3 & 6 & 1 \\
CEO, Management, Spokespeople & 3 & 1 & & & & \\
BP Employees and Contractors
\end{tabular}

\begin{tabular}{|c|c|c|c|}
\hline $\begin{array}{l}\text { Business and Corporate } \\
\text { Ol Industry (Transocean, Halliburton) } \\
\text { Ol industry Funded Hesearch and Non- } \\
\text { Profit } \\
\text { Non-Locad Business } \\
\text { Financial Industry } \\
\text { Other }\end{array}$ & 3 & 2 & 2 \\
\hline
\end{tabular}

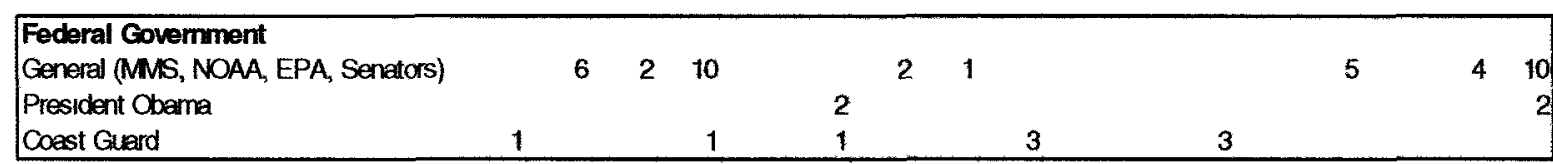

\begin{tabular}{|l|r|l|l|}
\hline BP and Government (quoted as one) & 1 & 1 & 1 \\
\hline
\end{tabular}

\begin{tabular}{|c|c|c|c|c|}
\hline \multicolumn{5}{|l|}{ Experts(Non-BP) } \\
\hline Scientist & 7 & & & \\
\hline \multicolumn{5}{|l|}{ Engneer } \\
\hline Biologist & 4 & & & \\
\hline \multicolumn{5}{|l|}{ Acadermic (non-al industry) } \\
\hline Energy/Petroleum Academic & & 1 & 2 & 1 \\
\hline Other & 1 & & & \\
\hline
\end{tabular}

Environmentalists

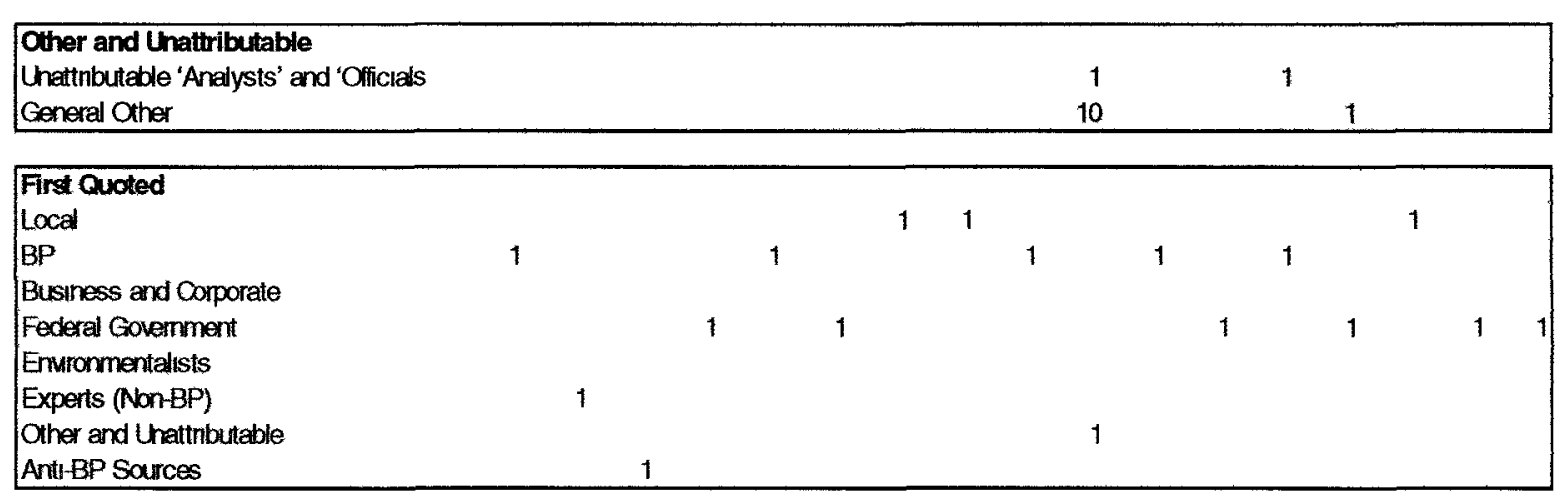




\section{Table Two: Total Quoted Per Category \\ Total Quoted}

Locals

Fishermen

General Locals

Local Politicians

53

29

Local Businesses/Business owners

Rig Worker

Local Attorney

Other (non-profit, land trust, other officials)

57

16

18

Total

BP

CEO, Management, Spokespeople

198

BP Employees and Contractors

163

Total

Business and Corporate

Oil Industry (Transocean, Halliburton)

Oil Industry Funded Research and Non-Profit

Non-Local Business

Financial Industry

Other

Total

Federal Government

General (MMS, NOAA,

President Obama

Coast Guard

Total

BP and Government (quoted as one)

Total

Antl-BP Sources

Anonymous Employees

Condemning documents

Total

Experts (Non-BP)

Scientist

Engineer

Biologist

Academic (non-oil industry)

Energy/Petroleum Academic

Other

Total

Environmentalists

Total

Other and Unattributable

Unattributable 'Analysts' and 'Officiais

General Other

Total

7
6
8
7
8
8




\begin{tabular}{|c|c|c|c|}
\hline & Total Quoted & $\begin{array}{l}\text { Quoted in } \\
\text { Number of } \\
\text { Articles }\end{array}$ & $\begin{array}{l}\text { Quoted in \% of } \\
\text { Total Articles }\end{array}$ \\
\hline Locals & 198 & 34 & 48.6 \\
\hline BP & 171 & 38 & 54.3 \\
\hline Business and Corporate & 77 & 27 & 38.6 \\
\hline Federal Government & 188 & 46 & 65.7 \\
\hline BP and Government & 10 & 9 & 12.9 \\
\hline Anti-BP Sources & 44 & 9 & 12.9 \\
\hline Experts (Non-BP) & 70 & 27 & 38.6 \\
\hline Environmentalists & 26 & 10 & 14.3 \\
\hline Other and Unattributable & 41 & 15 & 20 \\
\hline
\end{tabular}

Table Four: First Quoted by Category

Total Articles First Quoted

Local

Quoted First \%

$\mathrm{BP}$

$13 \quad 18.57$

Business and Corporate

$20 \quad 28.57$

Federal Government

$\begin{array}{rr}3 & 4.29 \\ 20 & 28.75\end{array}$

Environmentalists

Experts (Non-BP)

$\begin{array}{ll}3 & 4.29 \\ 6 & 8.57\end{array}$

Other and Unattributable

Anti-BP Sources

$\begin{array}{ll}3 & 4.29\end{array}$

Total

$\begin{array}{ll}2 & 2.89\end{array}$

70 


\section{Articles in the Sample}

Date Range One

April 21-252010

1. Oil Rig Sinks, Raising Fears Of a Major Spill in the Gulf - NYTimes.com

2. Search Continues After ()il Rig Blast - NYTimes.com

3. Search Ends for Missing Oil Rig Workers - NYTimes.com

4. Accidents Don't Slow Gulf of Mexico Drilling - NYTimes.com

Date Range Two

May 5-9 2010

5. The Tangled Question of Liability - NYTimes.com

6. Katrina Experiences Make Many Wary of Promises After Spill - NYTimes.com

7. Amount of Spill Could Escalate, Company Admits - NYTimes.com

8. Gulf Sees Familiar Face in Charge of Oil Spill Cleanup - NYTimes.com

9. Fast-Growing BP Also Has a Mounting List of Spills and Safety Lapses -

NYTimes.com

10. Workers on Oil Rig Recall a Terrible Night of Blasts - NYTimes.com

11. In Gulf of Mexico, Chemicals Under Scrutiny - NYTimes.com

12. As Oil Threat Creeps Closer, a Rush on Seafood - NYTimes.com

13. Clarifying Questions of I iability, Cleanup and Consequences - NYTimes.com

14. Federal Regulators' Warnings on Safety Weren't Acted On - NYTimes.com

16. Hair and Animal Fur Donated to Fight Oil Spill - NYTimes.com

17. From Air, BP's Chief Sees Progress in Containing Spill - NYTimes.com

18. Giant Container to Collect Leaking Gulf Oil - NYTimes.com

19. A New Setback in Efforts to Contain the Gulf Oil Spill - NYTimes.com

20. This Land - As the Oil Threatens, Lowering the Boom - NYTimes.com

21. Containment Effort Inches Closer to Target in Gulf - NYTimes.com

Date Range Three

May 29-June 22010

22. BP Says 'Top Kill' Failed to Stop Gulf Leak - NYTimes.com

23. BP Tries Again to Capture Oil With a Dome - NYTimes.com

24. Scientists Build Case for Undersea Plumes - NYTimes.com

25. Little Headway Made as BP Struggles to Halt Oil Leak - NYTimes.com

26. U.S. Opens Criminal Inquiry Into Oil Spill - NYTimes.com

27. Our Fix-It Faith and the Oil Spill - NYTimes.com

28. LEAK MAY PERSIST THROUGH AUGUST, OBAMA AIDE SAYS -

NYTimes.com

29. Documents Show Early Worries About Safety of Rig - NYTimes.com

30. A Waiting Game on the Louisiana Water-NYTimes.com

31. Criticism Over Speed and Care of Oil Spill Cleanup - NYTimes.com 
32. Island's Trout Rodeo Is Victim of Spill, and That's Not the Least of It NYTimes.com

33. Fishermen Fear Distuption of Their Way of Life - NYTimes.com

34. Oil Rig Official Says He Tried to Activate Emergency System - NYTimes.com

35. Cleanup Costs and Lawsuits Rattle BP's Investors - NYTimes.com

36. Administration Opens Inquiries Into Oil Disaster - NYTimes.com

37. Visiting Gulf, Obama Tells Residents 'You Are Nol Alone' - NYTimes.com

38. Billy Nungesser Becomes Face of Anger Over Spill - NYTimes.com

39. A Daunting Starl of Summer for 5 Gulf State Governors - NYTimes.com

40. Scientists Worry About Oil Plumes' Effects on Coral Reefs - NYTimes.com

Date Range Four

July 26-30 2010

41. BP Is Expected to Replace Chief With American - NYTimes.com

42. Dudley Promises to Look at BP's Culture and Safety - NYTimes.com

43. Gulf of Mexico Has Long Been Dumping Site - NYTimes.com

44. Gulf Surface Oil Vanishing Quickly - NYTimes.com

45. BP Details $\$ 30$ Billion Plan to Pay for Gulf Spill - NYTimes.com

46. Now, to Clean Up BP - NYTimes.com

47. Dudley to Take Over BP at a Critical Time - NYTimes.com

48. Lawmakers Offer Bills To Address Spill Issues - NYTimes.com

49. Delta's Black Fishermen Seeking Cleanup Work and Clinging to Hope -

NYTimes.com

Date Range Five

August 2-6 2010

50. Gulf Spill Is the Largest of Its Kind, Scientists Say - NYTimes.com

51. 'Static Kill' Of the Well Is Working, Officials Say - NYTimes.com

52. BP Begins 'Static Kill' - NYTimes.com

53. BP Done Pumping Cement Into Well - NYTimes.com

54. Oil in Gulf Poses Only Slight Risk, New U.S. Report Says - NYTimes.com

55. Coverage Turns, Cautiously, to Spill Impact - NYTimes.com

56. BP to Sell Colombian Unit for $\$ 1.9$ Billion - NYTimes.com

57. BP's Next Chief, Robert Dudley, Makes a Stop in Moscow - NYTimes.com

58. After Spill, Broad Anxiety Among Gulf Residents, Survey Finds - NYTimes.com

59. U.S. Puts Oil Spill Total at Nearly 5 Million Barrels - NYTimes.com

60. Oil Rig's Owner Had Safety Issue at Three Other Wells - NYTimes.com

61. Oil Spill Cleanup Workers Include Many Very, Very Small Ones - NYTimes.com

62. Oil Spill Donations Are Small, but Some Companies Step Up - NYTimes.com

63. Laid-Off Mexican Housekeepers Want BP to Help - NYTimes.com

64. In Gulf, Good News Is Taken With Grain of Salt - NYTimes.com

Date Range 6

September 7-11 2010

65. Hearing Records Detail Deepwater Horizon Blast - NYTimes.com

66. Final Sealing of BP Gulf Well Is Delayed Again - NYTimes.com 
67. BP Report Places Much Blame for Spill on Others - NYTimes.com

Date Range 7

Jan 5-11 2011 (Should be 4-10)

68. Oil Spill Panel Calls for Strict New Rules and New Spending - NYTimes.com

69. Gulf Spill Was Caused by Series of Errors, Panel Says - NYTimes.com

70. In Louisiana, Scope and Pace of Gulf Cleanup Is Criticized - NYTimes.com

71. Son of the Bayou. Torn Over the Shrimping Life - NYTimes.com 


\section{BIBLIOGRAPHY}

Andrews, Marcellus. 2009. "Burying Neoliberalism." Dissent 56(3): 57-62.

Anderson, Alison. 2002. "The Media Politics of Oil Spills." Spill Science and Technology Bulletin 7(1-2): 7-15.

Anderson, Alison. 2007. "Slick PR? The Media Politics of the Prestige Oil Spill." Science Communication 29(1): 96-115.

Antilla, L. 2005. "Climate of Skepticism: US Newspaper Coverage of the Science of Climate Change." Global Environmental Change 15:338-52.

Bell, Alan. 1995. "Language and the Media." Annual Review of Applied Linguistics 15: 23-41.

Bennett, Lance, Regina Lawrence and Steven Livingston .2007. When the Press Fails: Political Power and the News Media from Iraq to Katrina. Chicago: University of Chicago Press.

Brown, Jane Delano, Carl Bybee, Stanley Wearden and Dulcie Murdock Staughan. 1987. "Invisible Power: Newspaper News Sources and the Limits of Diversity." Journalism Quarterly 64: 45-54.

Callaghan, Karen and Frauke Schnell. 2001. "Assessing the Democratic Debate: How the News Media Frame Elite Policy Discourse." Political Communication 18: 183212.

Carlson, Matt. 2009. "Dueling, Dancing, or Dominating? Journalists and Their Sources." Sociology Compass 3(4): 526-542.

Carroll, William and Murray Shaw. 2001. "Consolidating a Neoliberal Bloc in Canada, 1976-1996." Canadian Public Policy 27(2): 195-216.

Castree, Noel. 2010. "Crisis, Continuity and Change: Neoliberalism, the Left and the Future of Capitalism." Antipode 41(1): 185-213.

Eagleton, Terry. 1991. Ideology: An Introduction. New York: Verso.

Entman, Robert. 2002. "Framing: Towards Clarification of a Fractured Paradigm" in McQuail's Reader in Mass Communication Theory, ed. Denis McQuail. London: Sage Publications.

-----2007. "Framing Bias: Media in the Distribution of Power." Joumal of Communication 57(1): 163-173. 
Ericson, Richard, Patricia Baranek and Janet Chan. 1987. Visualizing Deviance: A Study of News Organizations. Toronto: University of Toronto Press.

Fairclough, Norman. 1995 a. Critical Discourse Analysis: The Critical Study of Language. New York: Longman.

-1995 b. Media Discourse. London: Edward Arnold.

-----2001. Language and Power. 2nd ed. New York: Longman.

-----2003. Analysing Discourse Textual Analysis for Social Research. London: Routledge.

Fiske, J. 1994. Media Matters: Everyday Culture and Political Change. Minneapolis: University of Minnesota Press.

Flyvbjerg, Bent. 2001. Making Social Science Matter. New York, NY: Cambridge University Press.

-----2005. "Social Science That Matters." Foresight Europe No. 2 (October 2005 March 2006): 38-42.

Frank, Arthur. 2002. "Why Study People's Stories? The Dialogical Ethics of Narrative Analysis." International Journal of Qualitative Methods 1(1): 1-19.

Freudenburg, William and Robert Gramling, 2011. Blowout in the Gulf. Cambridge: MIT Press.

Gamson, William and Andre Modiliani. 1989. "Media Discourse and Public Opinion: A Constructionist Approach." American Journal of Sociology 95(1): 1-97.

Gans, Herbert. 1979. Deciding What's News: A Study of CBS Evening News, NBC Nightly News, Newsweek and Time. New York: Pantheon Books.

Gitlin, Todd. 2003. The Whole World is Watching: Mass Media Making and Unmaking of the New Left. Berkeley: University of California Press.

Goffman, Erving. 1974. Frame Analysis: An Essay on the Organization of Experience. New York: Harper Colophon Books.

Gorman and Seidman. 2010. "TV by the Numbers." Cable News Ratings. Retrieved on September 20, 2010 from: http://tvbythenumbers.com/category/ratings/cable-news

Gray, David E. 2004. Doing Research in the Real World. Thousand Oakes: Sage Publications. 
Greenpeace. 2010. "Greenpeace Response to National Oil Commission Final Report." Greenpeace USA. Retrieved on June 242011 from: http://www.greenpeace.org/usa/en/media-center/news-releases/Greenpeaceresponse-to-National-Oil-Spill-Commission-final-report/

Hall, Stuart, Chas Critcher, Tony Jefferson, John Clarke and Brian Roberts. 1978. Policing the Crisis: Mugging, the State and Law and Order. London: MacMillan.

Hallin, Daniel. 2008. "Neoliberalism, Social Movements and Change in Media Systems in the Late Twentieth Century." in The Media and Social Theory, David Hesmondhalgh and Jason Toynbee, eds. New York: Routledge.

Hannigan, John. 2006. Environmental Sociology. London: Routledge.

Hansen, Kathleen. 1991. "Source Diversity and Newspaper Enterprise Journalism." Joumalism Quarterly 68: 474-82.

Harvey, David. 2007. A Brief History of Neoliberalism. New York: Oxford University Press.

Herman, Edward and Noam Chomsky. 2002. Manufacturing Consent: The Political Economy of the Mass Media. New York: Pantheon Books.

Ivengar, Shanto, Donald Kinder. 1988. News that Matters: Television and American Opinion. Chicago: University of Chicago Press.

Jewkes, Yvonne. 2004. Media and Crime. Thousand Oakes, CA: Sage Publications.

Joling, Dan and Mark Thiessen. 2010. "Exxon Valdez Victims Give Gulf Region Oil Spill Advice: Describe Shocking Toll of Human Disaster." The Huffington Post. Retrieved on September 152011 from: http://www.huffingtonpost.com/2010/05/03/exxon-valdez-victimsgive_n_560624.html

Keil, Roger. 2002. "Common-Sense' Neoliberalism: Progressive Conservative Urbanism in Toronto, Canada." Antipode 34(3): 578-601.

Kimball, Penn. 1994. Downsizing the News: Network Cutbacks in the Nations Capital. Baltimore: John Hopkins University Press.

Kirsch, Stuart. 2010. "Guest Editorial: Sustainability and the BP Oil Spill." Dialectical Anthropology. 34(3): 295-300.

Koenig, Thomas. 2006. "Compounding Mixed-Methods Problems in Frame Analysis Through Comparative Research." Qualitative Research. Thousand Oakes, CA: Sage Publications. 
Lipmann, Walter. 1965. "The Nature of News." Public Opinion. New York: Free Press.

Markey, Edward. 2010. "Waking up From the BP Nightmare." Retrieved on August 29, 2011 from: http://www.huffingtonpost.com/rep-ed-markey/waking-america-fromthe-b b $613161 . \mathrm{html}$

Marx, Karl, Friedrich Engels Ernest Untermann, Samuel Moore and Edward Aveling. 1906-1909. Capital: A Critique of Political Economy, Chicago: C. H. Kerr \& Company.

McChesney, Robert W. March 2001. "Global Media, Neoliberalism and Imperialism." Monthy Review 52(10).

McCombs, Maxwell E. and Donald L. Shaw. 1972. "The Agenda Setting Function of Mass Media." Public Opinion Quarterly 36(2): 176-187.

McQuail, D. 1994. Mass Communication Theory: An Introduction. Thousand Oaks: Sage Publications.

Mene, Aditi. 2011. "The Gulf of Mexico Oil Spill: Consequences for the Oil and Gas Industry." Machodo Meyer. Retrieved on August 29, 2011 from: http://www.machadomeyer.com/news/the-Gulf-of-mexico-oil-spill\%3Aconsequences-for-the-oil-and-gas-industry

Mills, C. Wright. 1956. The Power Elite. New York: Oxford University Press.

Molotch, Harvey and Marilyn Lester. 1975. "Accidental News: The Great Oil Spill as Local Occurance and National Event." American Journal of Sociology 81(2): 235260.

MSNBC. 2010. "Oil spill timeline." Online Database of Daily News. Retrieved on September 15, 2010 from: http://www.msnbc.msn.com/id/36947751/).

Mudge, Stephanie L. 2008. "What is Neo-Liberalism?" Socio-Economic Review 6: 703731.

Mulvale, James. 2001. Reimagining Social Welfare: Beyond the Keynesian Welfare State. Toronto: Garamond Press.

Munck, Ronaldo. June 2006. "Globalization and Contestation: A Polanyian Problematic." Globalizations 3(2): 175-186.

Newspaper Association of America. 2011. Retrieved on August 12. 2011 from: http://www.naa.org 
Oliver, Pamela and Hank Johnston. 2000. "What a Good Idea! Ideologies and Frames in Social Movement Research." Mobilization: An International Quarterly 5(1): 3754.

Peck, Jamie, Nick Theodore and Neil Brenner. January 2010. "Postneliberalism and its Malcontents." Antipode Supplement 1(41): 94-116.

Philo, Greg. "New Content Studies, Media Group Methods and Discourse Analysis: A Comparison of Approaches."

PETEX. 2011. "About Us: The Petroleum Extension Service." Retrieved on August 24 2011 from http://www.utexas.edu/ce/petex/about/

Polyani, Karl. 1944. The Great Transformation. Boston: Beacon.

Postman, Neil. 1985. Amusing Ourselves to Death: Public Discourse In the Age of Show Business. New York: Penguin.

Purvis, Trevor and Allen Hunt. 1993. "Discourse, Ideology, Discourse, Ideology, Discourse, Ideology..." British Journal of Sociology 44(3): 473-497.

Safina, Carl. 2011. A Sea in Flames. New York: Random House.

Schudson, Michael. 1989. "The Sociology of News Production." Media, Culture and Society 11: 263-282.

Scheufele, Dietram. 1999. "Framing as a Theory of Media Effects." Journal of Communication Winter 1999: 103-122.

Scheufele, Dietram and David Tewksbury. 2007. "Framing, Agenda Setting, and Priming: The Evolution of Three Media Effects Models." Journal of Communication 57(1): 9-20.

Semetko, Holli and Patt Valkenburg. 2000. "Framing European Politics: A Content Analysis of Press and Television News." Journal of Communication Spring 2000: 93-109.

Sokari. 2010. "Niger Delta: Oil Spills in Perspective." Black Looks. Retrieved on September 3, 2011 from: http://www.blacklooks.org/2010/05/niger-delta-oil-spillsin-perspective/

Stelter, Brian. October 14 2009. "White House vs. Fox News, Not Just Fox Opinion." New York Times Online. Retrieved on October 262010 from: http://mediadecoder.blogs.nytimes.com/2009/10/14/white-house-vs-fox-news-notjust-fox-opinion/ 
Treanor, Paul. 2004. Neoliberalism: Origins, Theory, Definition. Retrieved August 18 2011 from: http://web.inter.nl.nct/users/Paul.Treanor/neoliberalism.html

Toolan, Michael. 2001. "Narrative as Political Action." In Narrative: A Critical Linguistic Introduction. Florence, KY, Routledge, Ch. 8

Vidal, John. 2010."Nigeria's Agony Dwarfs the Gulf Oil Spill: The US and Europe Ignore It." The Guardian Observer. Retrieved on August 282011 from: http://www.guardian.co.uk/world/2010/may/30/oil-spills-nigeria-niger-delta-shell

Young, Nathan and Eric Dougas. 2011. "Representations of Climate Change in Canadian National Print Media: The Banalization of Global Warming." Canadian Review of Sociology 48(1): 1-22.

Wardell, Jane. 2010. "BP CEO Hayward Tested by the Gulf of Mexico Spill." MSNBC. Retrieved on July 212011 from: http://www.msnbc.msn.com/id/36958500/ns/business-world_business/t/bp-ceohayward-tested-Gulf-mexico-spill/

Weaver, David. 2007. "Thoughts on Agenda Setting, Framing and Priming." Journal of Communication. 57(1): 142-147.

Weber, Max. 1992. From History to Modernit. New York: Routledge.

Whitty, Julia. 2010. "The BP Cover-Up." Mother Jones. Retrieved on August 14, 2010 from: http://motheriones.com/environment/2010/09/bp-ocean-cover-up

Wodak, Ruth and C Ludwig. (Eds). 1999. Challenges in a changing world: Issues in critical discourse analysis. Vienna: Passagenverlag. 